\title{
Advanced Progress of Optical Wireless Technologies for Power Industry: An Overview
}

\author{
Jupeng Ding ${ }^{1, * \mathbb{C}}$, Wenwen Liu ${ }^{1}{ }^{\mathbb{C}}$, Chih-Lin $\mathrm{I}^{2}$, Hui Zhang ${ }^{3}$ and Hongye Mei ${ }^{1}$ \\ 1 Key Laboratory of Signal Detection and Processing in Xinjiang Uygur Autonomous Region, \\ School of Information Science and Engineering, Xinjiang University, Urumqi 830046, Xinjiang, China; \\ wenwenlaua@163.com (W.L.); meihongyesss@163.com (H.M.) \\ 2 China Mobile Research Institute, Beijing 100053, China; icl@chinamobile.com \\ 3 Xinjiang Vocational \& Technical College of Communications, Urumqi 831401, Xinjiang, China; \\ zh007red@163.com \\ * Correspondence: jupeng7778@163.com
}

Received: 12 August 2020; Accepted: 9 September 2020; Published: 16 September 2020

\begin{abstract}
Optical wireless communications have attracted widespread attention in the traditional power industry because of the advantages of large spectrum resources, strong confidentiality, and freedom from traditional electromagnetic interference. This paper mainly summarizes the major classification and frontier development of power industry optical wireless technologies, including the indoor and outdoor channel characteristics of power industry optical wireless communication system, modulation scheme, the performance of hybrid power line, and indoor wireless optical communications system. Furthermore, this article compares domestic and foreign experiments, analyzes parameters for instance transmission rate, and reviews different application scenarios such as power wireless optical positioning and monitoring. In addition, in view of the shortcomings of traditional power technology, optical wireless power transfer technology is proposed and combined with unmanned aerial vehicles to achieve remote communication. At last, the main challenges and possible solutions faced by power industry wireless optical technologies are proposed.
\end{abstract}

Keywords: optical wireless technologies; power industry; power line communications; indoor wireless optical communications; optical wireless power transfer

\section{Introduction}

Optical wireless communications (OWC), have many advantages such as large spectrum resources, strong confidentiality, and freedom from traditional electromagnetic interference. Therefore, it has been widely concerned by the habitual industry represented by the electric power field. The further deepening of the integration of various emerging information technologies and the power grid, various optical wireless technology solutions that rely on and serve smart power have been proposed successively and formed a preliminary scale. Optical wireless technologies are mainly classified into two categories: outdoor wireless optical communication, which is often called free space optical (FSO) technology, and indoor wireless optical communications (IWOC), which is regarded as visible light communications (VLC) because of using visible light as the emitter light [1-6]. Specifically, Table 1 provides specific parameter indicators to help better understand the differences between the two types of technologies [7-10]. It can be seen from Table 1 that the most obvious disadvantage of IWOC is that it is not suitable for outdoor applications because of its short communication distance [7], while outdoor OWC is suitable for long-distance transmission due to its transmission distance up to tens of thousands of kilometers despite environmental interference [7]. 
Table 1. Classification parameters of optical wireless communications [7-9].

\begin{tabular}{|c|c|c|c|c|c|c|c|}
\hline Types & Transmitter & Receiver & Distance & Data Rate & Spectrum & Main Limits & Ref \\
\hline $\begin{array}{l}\text { Indoor } \\
\text { (VLC) }\end{array}$ & LED/LD ${ }^{1}$ & $\mathrm{PD}^{2} /$ camera & $10 \mathrm{~m}$ & $\begin{array}{c}10 \mathrm{Gbps} \\
\text { (LED)/100Gbps } \\
\text { (LD) }\end{array}$ & VL & $\begin{array}{l}\text { 1. Short distance } \\
\text { communication; } \\
\text { 2. Not suitable } \\
\text { outdoor; } \\
\text { 3. No guaranteed of } \\
\text { mobility support }\end{array}$ & {$[7,8]$} \\
\hline $\begin{array}{l}\text { Outdoor } \\
\text { (FSO) }\end{array}$ & LD & PD & $\begin{array}{l}\text { More than } \\
10,000 \mathrm{~km}\end{array}$ & 40 Gbps & $\mathrm{IR} / \mathrm{VL} / \mathrm{UV}^{3}$ & $\begin{array}{c}\text { Environment } \\
\text { dependent }\end{array}$ & {$[7,9]$} \\
\hline
\end{tabular}

${ }^{1}$ LD stands for laser diode. ${ }^{2}$ PD is for Photodetector. ${ }^{3} \mathrm{IR}$, VL, and UV represent for infrared, visible light and ultraviolet, which their wavelength ranges are $760 \mathrm{~nm}-1 \mathrm{~mm}, 360-760 \mathrm{~nm}$ and $10-40 \mathrm{~nm}$ [7].

Optical wireless technologies with the traditional power of the intersection of information technology can be traced back to 2003. Researchers at Japan's Keio university took into account the characteristics of white light-emitting diode (LED): high brightness, low power consumption, and long service life etc., and based on the superior properties, first proposed the white LED IWOC and traditional power line communications (PLC) integrated system solutions [1]. This scheme has the inherent advantage that IWOC is free from the traditional radio frequency (RF) electromagnetic interference and the LED light source has high radiation power. Furthermore, the scheme can reduce the construction cost to a greater extent by reusing existing power line infrastructure and avoiding the introduction of new communications cables. At the same time, the above team also presented the prototype system implementation of the proposed scheme, which uses a desk lamp equipped with 120 LED as the IWOC light source and adopts sub-carrier binary shift keying (SC-BPSK). In addition, in the system of compact design, the team could apply to existing ball steep light screw is given and at the same time meet the requirements of the proposed scheme of bulb type IWOC transmitter components.

This groundbreaking work has aroused the enthusiasm of many domestic and international research teams. So far, researchers from the University of Johannesburg in South Africa, the University of Texas in the United States, the University of Southampton in the United Kingdom, the University of British Columbia in Canada, Tsinghua University and the Information Engineering University of the People's Liberation Army have participated in the research work in this interdisciplinary field, and the research results have begun to take shape [10-17].

At present, the research directions in this field mainly include integrated channel characteristics and transmission scheme of PLC and IWOC, modulation schemes, the performances of hybrid PLC and IWOC system, and optical wireless monitoring of high-voltage lines and inspection. This paper will sort out the development of optical wireless technologies for power industry in recent years about the above directions, and demonstrate the frontier progress in this field by combining with a number of experimental prototypes. The main challenges and possible solutions for power industry OWC are summarized to provide useful guidance for the further development of the technology.

With the development of science and technology, power industry optical wireless technologies are widely used in positioning, monitoring, and people's daily life. The application scenarios of power industry optical wireless technologies are shown in Figure 1. Figure 1a gives the indoor scenario that PLC and IWOC combine for smart home. The tunnel inspection is as shown in Figure 1b. The inspector communicates with the LED through a handheld device and transmits detection signals to the data center through the PLC link. The outdoor street lamp monitoring is as shown in Figure 1c, which is used to prevent traffic accidents. In addition, Figure $1 \mathrm{~d}$ shows the hospital monitoring system, which could monitor the patient's physical health in real time. Furthermore, Figure 2 lists the block diagram of the power industry optical wireless technologies, including technical classifications, modulation methods, and application scenarios. 


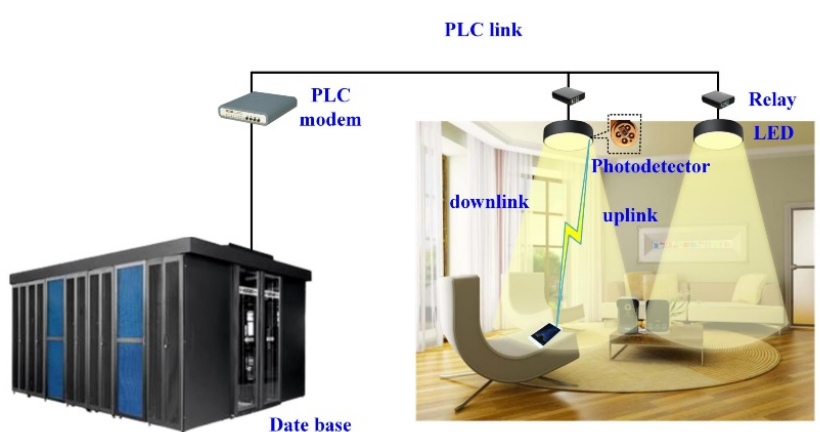

(a)

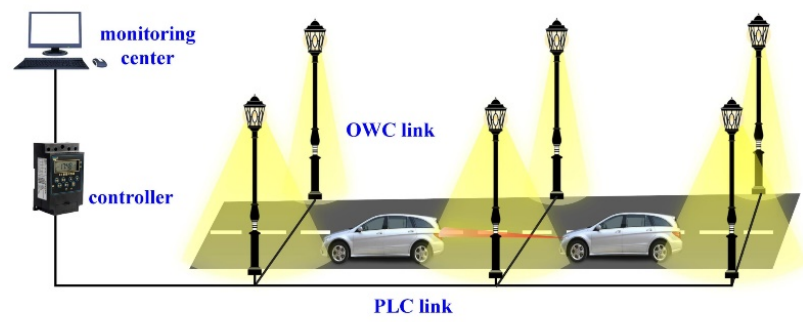

(c)

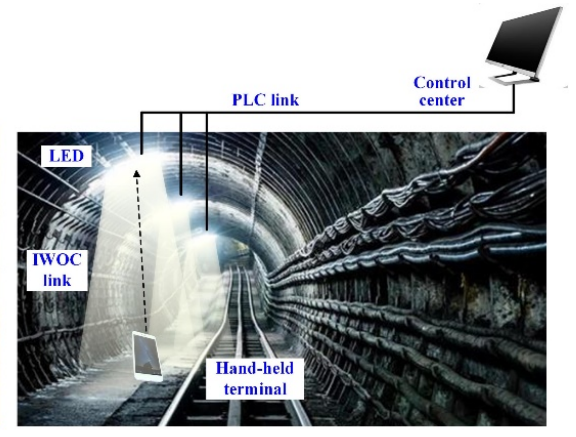

(b)

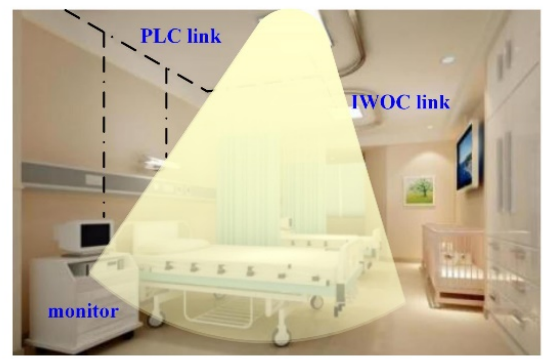

(d)

Figure 1. The application scenarios of power industry optical wireless systems: (a) indoor scenario; (b) tunnel scenario; (c) outdoor scenario; (d) hospital scenario.

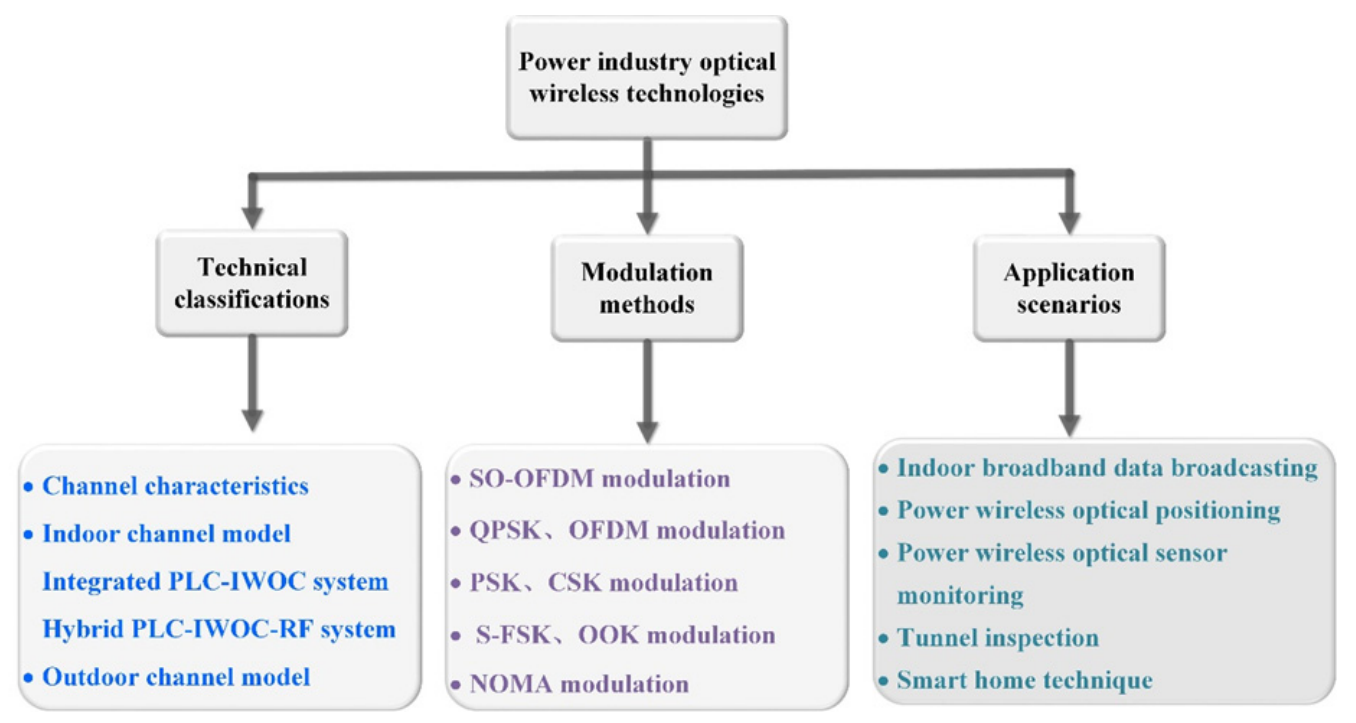

Figure 2. Block diagram of the power industry optical wireless technologies.

\section{Classification and Progress of Power Industry Optical Wireless Communications}

\subsection{Channel Characteristics between Power Line and Indoor Wireless Optical Communications}

In recent years, researchers have carried out a series of theoretical modeling and measurement activities for the channel characteristics of the power line and IWOC system in [18-22]. The block diagram for an integrated system is illustrated in Figure 3. In this system, the information signal is influenced by the inherent noise of PLC and IWOC channel successively. The module after pre-compensation and signal amplification, and then the data signal to the LED on the DC bias to drive LED light, which is as light transmitter coverage indoor areas. From an integration and commercial point of view, the module is very simple to install on the LED light and could be transformed into a communication device immediately. The light illuminated by the LED is received by the avalanche 
photodiode (APD), and then the APD amplifies and demodulates the signal according to the received light signal level. Taking into account the structure of the entire system, IWOC is used as the downlink. Consider an infrared adapter or PLC uplink, which will be very suitable for multimedia data services [19].

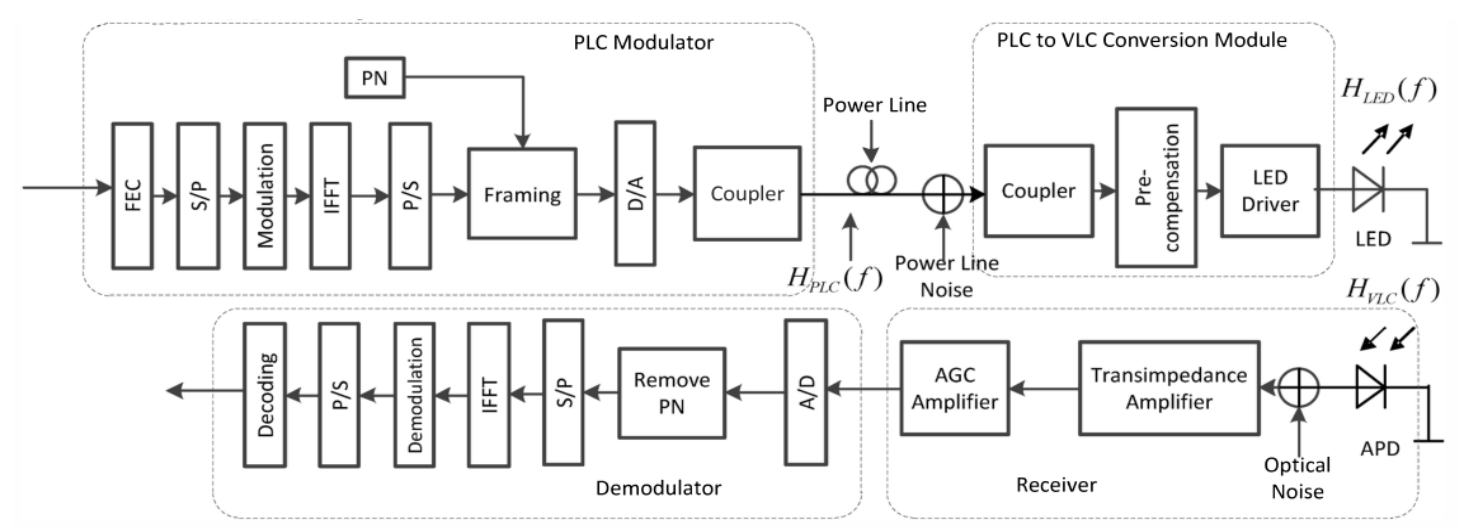

Figure 3. The block diagram of the integrated indoor wireless optical communications-power line communications (IWOC-PLC) system [19].

In [19], the signal is first modulated into an analog signal by the PLC modulator. The modulated signal is transmitted to the power line through the coupler. The system uses "PLC to IWOC conversion" module, as is shown in Figure 4, which is in the LED lamp to receive the data signal from the power cord.

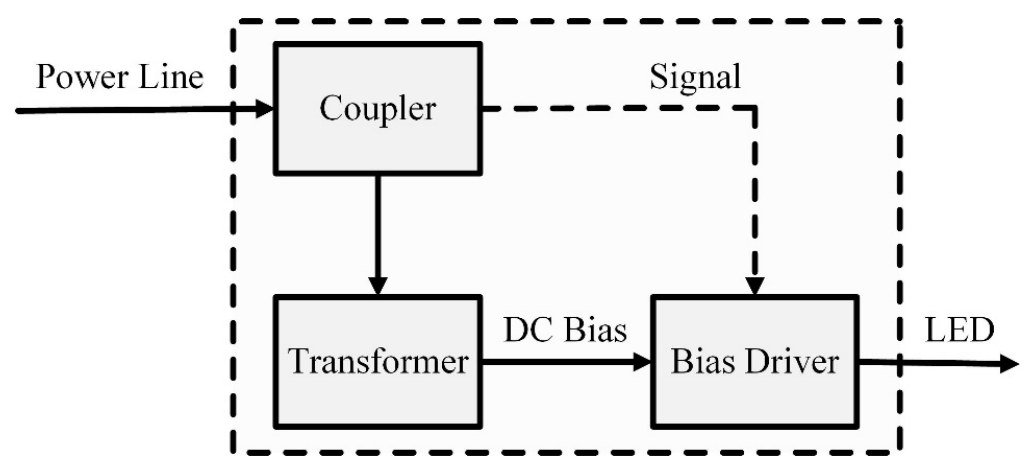

Figure 4. PLC to IWOC module [19].

\subsubsection{Channel Characteristics of Indoor Wireless Optical Communications}

The channel frequency response (CFR) of PLC is as shown in the following $[20,23]$ :

$$
H_{P L C}(x)=\sum_{l=1}^{N} g_{l} e^{-\left(\alpha_{0}+\alpha_{1} x\right) \tau_{l} v_{p}} e^{-j 2 \pi x \tau_{l}}
$$

where $g_{l}$ is for the weighting factor of the $l$ th path, which includes the reflection factor and transmission factor along the path, attenuation parameters are represented by $\alpha_{0}$ and $\alpha_{1}$, the exponent of the attenuation factor is expressed in terms of $x$ (usually 0.2 to 1 ), $v_{p}$ is for the group velocity and $\tau_{l}$ stands for the $l$ th path delay, and the $N$ denotes path number.

The received signal in the IWOC part can be expressed as:

$$
H_{V L C}(x)=S h_{L O S} e^{-j 2 \pi x t_{L O S}}
$$


where the discrete Fourier transform of the $h_{V L C}(x)$ is $H_{V L C}(x), S$ is the photosensitivity, the gain is expressed in terms of $h_{L O S}$, and $t_{L O S}$ represents the delay of the LOS signal, which is line of sight signal. In other words, there are no obstructions in the signal transmission process from the transmitter to the receiver.

\subsubsection{Channel Characteristics of Outdoor Wireless Optical Communications}

Outdoor transmission channel is atmospheric channel, which contains a large amount of gases and scattered particles, which will produce deviation in the transmission process of light. Some components and particles in the atmosphere will also absorb the energy of light, so that the transmitting power of the transmitted signal will be attenuated. These factors have a great negative effect on outdoor wireless optical communications system. After the OWC system equipment receives the signal through the power line, it is received through the optical interface, and connected to the receiving end through the optical cable, and is generally not affected by lightning strikes. The power supply voltage of wireless optical communication equipment is generally $-48 \mathrm{~V}$ DC or $220 \mathrm{~V} \mathrm{AC}$, and then converted to $12 \mathrm{~V}$ working voltage through the internal power module. The interference of the background light has a remarkable influence on the optical receiver. However, under the interference of intense natural light, especially under direct sunlight, the photodetector may enter a saturated state, causing the photodetector to fail to receive weak communication light. Atmospheric turbulence effect is due to the existence of air vortices with different temperature, pressure, density, flow velocity, and size in the atmosphere. These vortices collide with each other and superpose to form irregular atmospheric turbulence motion in the continuous movement, which results in the change of atmospheric refractive index, and makes visible light signal produce light intensity scintillation and central beam drift. Compared with the atmospheric attenuation effect, the atmospheric turbulence effect will randomly lead to the intensity fluctuation of the optical signal, so the influence of atmospheric turbulence is mainly considered in the analysis of outdoor visible optical communication channel.

Weather is crucial to communications when it comes to outdoor communications. The transmittance of visible light decreases in rain, snow, fog, and haze. Snowflakes, rain drops, and fog drops scatter and absorb visible light, etc. The optical signal transmitted through the atmosphere attenuates and the attenuation amplitude varies greatly, which seriously affects the transmission of light and the communication quality of the system. Therefore, channel conditions should be fully considered in LED outdoor visible light communication system to reduce the influence of external interference and improve system performance. Optical wireless (OW) transmission quality is very sensitive to weather. Sunny days have the least influence on OW transmission quality, while rainy days, snow days, and fog days have the greatest influence on wireless optical transmission quality. According to the test, the empirical values of optical wireless attenuation affected by weather are as follows: sunny days is $5-15 \mathrm{~dB} / \mathrm{km}$, rainy days is $20-50 \mathrm{~dB} / \mathrm{km}$, snow day is $50-150 \mathrm{~dB} / \mathrm{km}$, and foggy days is $50-300 \mathrm{~dB} / \mathrm{km}$ [24]. OWC technologies need to overcome the influence of weather on communications quality in order to meet the requirement of all-weather operation of power system communications.

Different from PLC, the reasons affected the quality of wireless communications are that the wireless penetration is weak and they propagate in the process of various obstacles. Therefore, the signals will be reflected, diffracted, and scattered or directly absorbed during the transmission process, and then the wireless signals are weakness. The wireless channel model can be divided into large-scale fading and small-scale fading [10].

Large-scale fading is usually divided into two categories: one is the fading of signal caused by the influence of other objects in the environment, which is called shadow fading, and the other is the transmission loss caused by signal communication over long distances, which is called path loss.

Small-scale fading is mainly due to the multipath signal at the receiving end passing through different paths during transmission, which leads to different amplitudes, delays, and phases. When the signal arriving at the receiving end has no LOS transmission, it is regarded as to obey the Rayleigh distribution. 
In the part of PLC, researchers usually follow the adaptive echo model put forward by Zimmermann [10]. This model comprehensively reflects the attenuation characteristics of multipath propagation, dependent frequency, and line length in a dynamic line network. In the IWOC part, for the convenience of modeling and analysis, researchers usually use the wireless optical integrator model to represent the frequency response of IWOC channel. Unlike the traditional OW non-parametric modeling scheme that requires data estimation to obtain the density distribution form, the channel gain of channel irradiance component can be obtained directly only by the surface area of the light receiver and the room and the average reflective of the room, and the modeling complexity is significantly reduced. By changing PLC channel parameters, the researchers simulated different PLC environments and frequency responses. With the increase in the number of PLC channel taps, the frequency response grooves of PLC and IWOC joint channels will need to be further increased.

The results of different PLC network with IWOC-PLC channel are shown in Figure 5 [11]. Figure 5a indicates the frequency response to IWOC-PLC channel. It evaluates a bad PLC network environment with six taps and bandwidth in excess of $10 \mathrm{MHz}$. The parameters of IWOC environment are a room with an area of about $25 \mathrm{~m}^{2}$, the effective surface area of IWOC receiver is $3 \mathrm{~cm}^{2}$, and the distance from the LED transmitter is about $3 \mathrm{~m}$ [11].

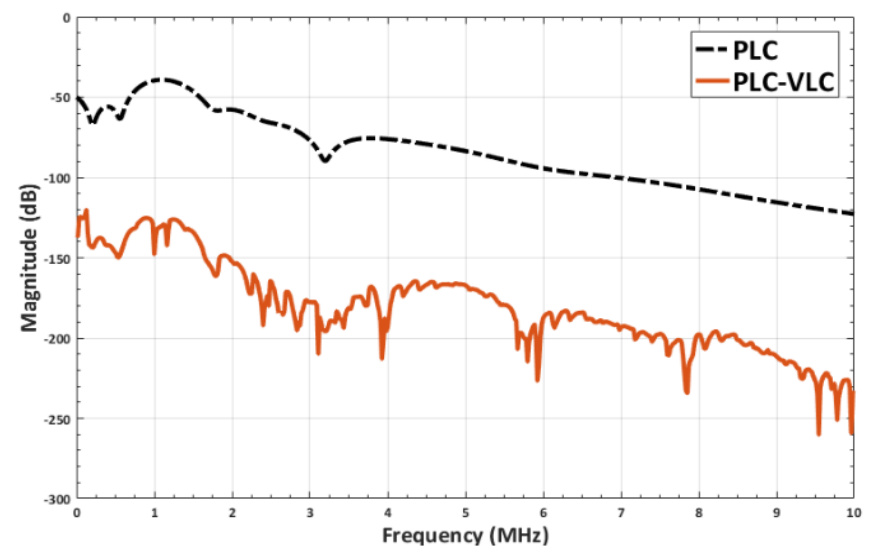

(a)

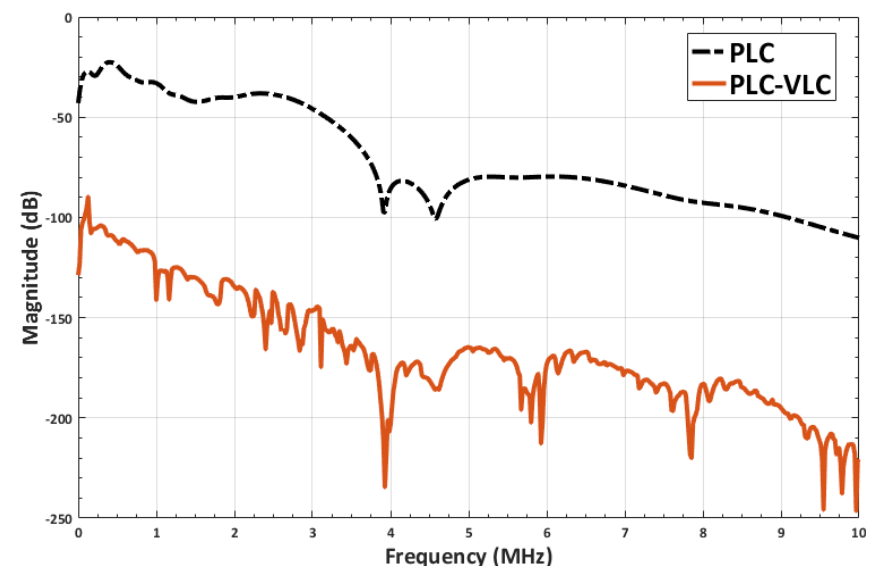

(b)

Figure 5. The results of different PLC network with their resulting IWOC-PLC channel: (a) 6 PLC taps network [11]; (b) 12 PLC taps network [11].

Figure 5 shows the PLC channel and the resulting IWOC-PLC channel. From the results, the PLC-IWOC channel has a notch characterized which varies throughout the entire frequency band. We have likewise observed the effect of the IWOC channel, which weakens frequency response 
of the PLC channel. IWOC-PLC channel also simulates and approximates the low-pass frequency characteristics of the PLC channel. Specifically, Figure $5 b$ shows the joint channel characteristics of PLC and IWOC with 12 PLC taps. In particular, the VLC in Figure 5a,b represented by IWOC in this paper.

Gao et al. studied the random channel generator of the hybrid PLC and IWOC system, and a set of variable parameters in [25] is used to describe the complex transfer function of the general typical apartment complex system network. The power line (with branch) is the backbone network. IWOC LED is used as a visible light modulator for electrical signal scheduling, as shown in Figure 6a.

In [26], the CFR of the indoor power line channel is presented as the sum of all propagation paths under low-voltage conditions. APD is a photoelectric converter that demodulates an optical signal into an electrical signal and is generally considered to be an all-pass filter because of its broadband properties. Figure $6 \mathrm{~b}$ shows the measured pure power line channels and integrated channels. It can be seen from the figure that, compared to pure PLC channel, the attenuation of the integrated channel is large and the channel performance is poor [25].

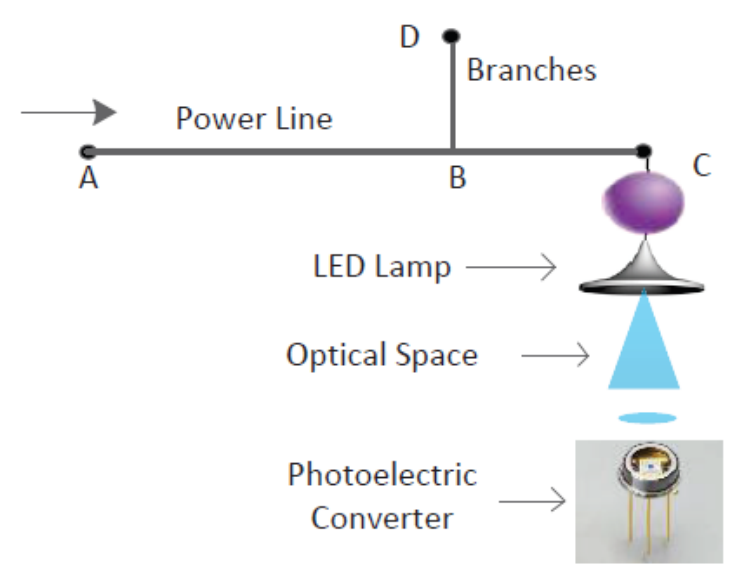

(a)

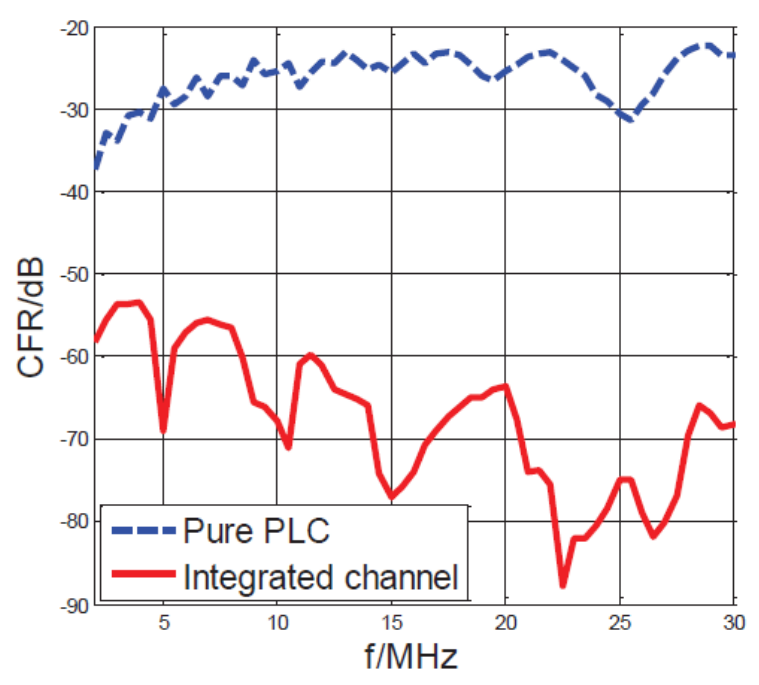

(b)

Figure 6. The integrated PLC and IWOC: (a) Channel structure of the integrated PLC and IWOC; (b) measured pure PLC channel and integrated channel [25].

To characterize the influence of LED modulation bandwidth on the frequency response of integrated PLC and IWOC channel, the researchers measured part of the frequency response of IWOC 
and the overall frequency response characteristics of the mixed system in the laboratory environment, as shown in Figure 7.

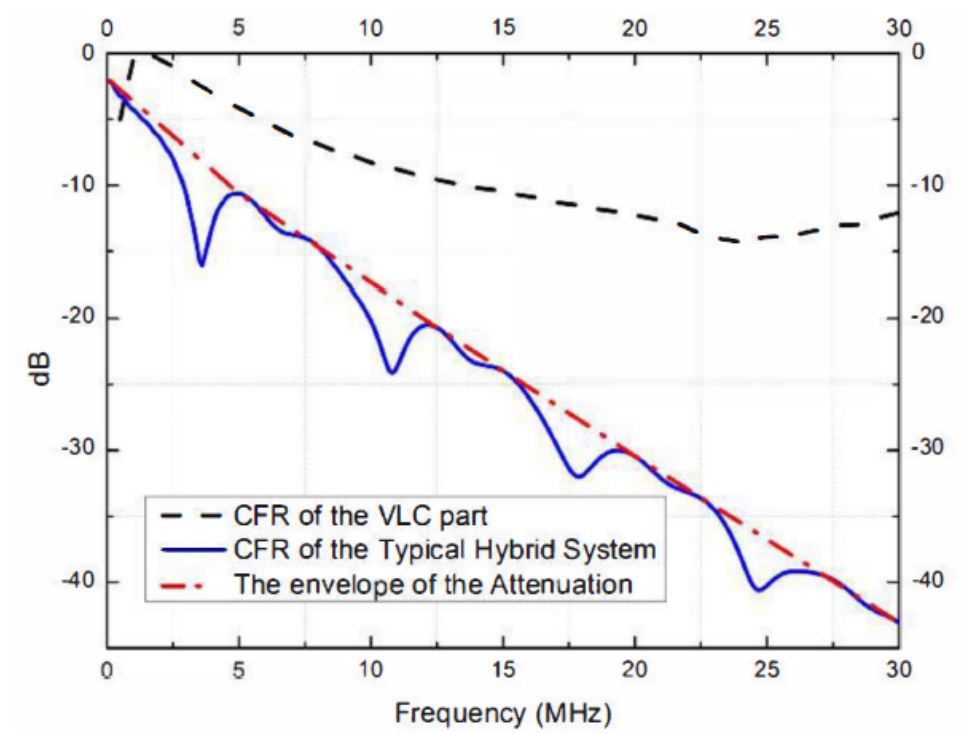

Figure 7. The measured channel frequency response (CFR) of a typical integrated PLC and IWOC system [19].

The measurement results are demonstrated that the attenuation introduced by the LED light source near $50 \mathrm{MHz}$ is only $10 \mathrm{~dB}$. However, once the IWOC system is intended to be integrated into the PLC system, the attenuation of the overall system at high frequencies will significantly reduce the available bandwidth of the system. Specifically, PLC channel uses a typical 6-diameter model, while the IWOC channel filters out stray light with blue light filters. Quantitative results show that the attenuation of the hybrid system is about $40 \mathrm{~dB}$ at $30 \mathrm{MHz}$, indicating that the available modulation bandwidth of the system will be severely limited.

\subsection{Modulation Methods of Hybrid Optical Wireless Communications}

Orthogonal frequency division multiplexing (OFDM) is a kind of modulation technology with high spectral efficiency, which can provide a simple solution for channel dispersion and can be implemented in the digital domain. OFDM can divide the available bandwidth of the channel into several sub-channels, and realize frequency division multiplexing through the orthogonality among sub-channels. It can also realize signal transmission and adapt to the adjustment conditions of the channel by using the allocation of bit and power on the subcarrier. It reduces the subcarrier transmission rate and prolongs the period of the code element, so it has excellent anti-multipath effect performance and realizes the high-speed transmission rate under the condition of limited bandwidth. In addition, combined with multi-input and multi-output (MIMO) technology, it is required that the receiver can receive the optical signal from the LED lamp array in order to resolve multiple independent communication channels. The parallel transmission of multiple transmitting and receiving units could improve the performance of IWOC and make different users occupy non-overlapping subcarrier sets, therefore realizing downlink multi-user transmission.

\subsubsection{Hybrid Wireless Optical System in OFDM Modulation}

In view of the fact that multiple light sources with visible light communication functions overlap the service area, S. Hranilovic research team from Canada proposed collaborating with and provide data for IWOC emitters through power lines. Specifically, they proposed a system architecture named hybrid IWOC-PLC, and gave the corresponding analytical framework $[14,27,28]$. In this scheme, 
researchers apply spatial optical-orthogonal frequency division multiplexing (SO-OFDM) to multiple sources, and propose an OFDM scheme to explore frequency selectivity of IWOC and PLC channels, as shown in Figure 8 [14,23]. Compared with the integration of traditional IWOC and PLC, this scheme improves the peak to average power ratio (PAPR) problem caused by the application of OFDM to LED emitter, and overcomes the interference between different light sources through the cooperation between spatial distributed light sources. In the meantime, the robustness of the system against local link occlusion is enhanced.

LED Luminary 1

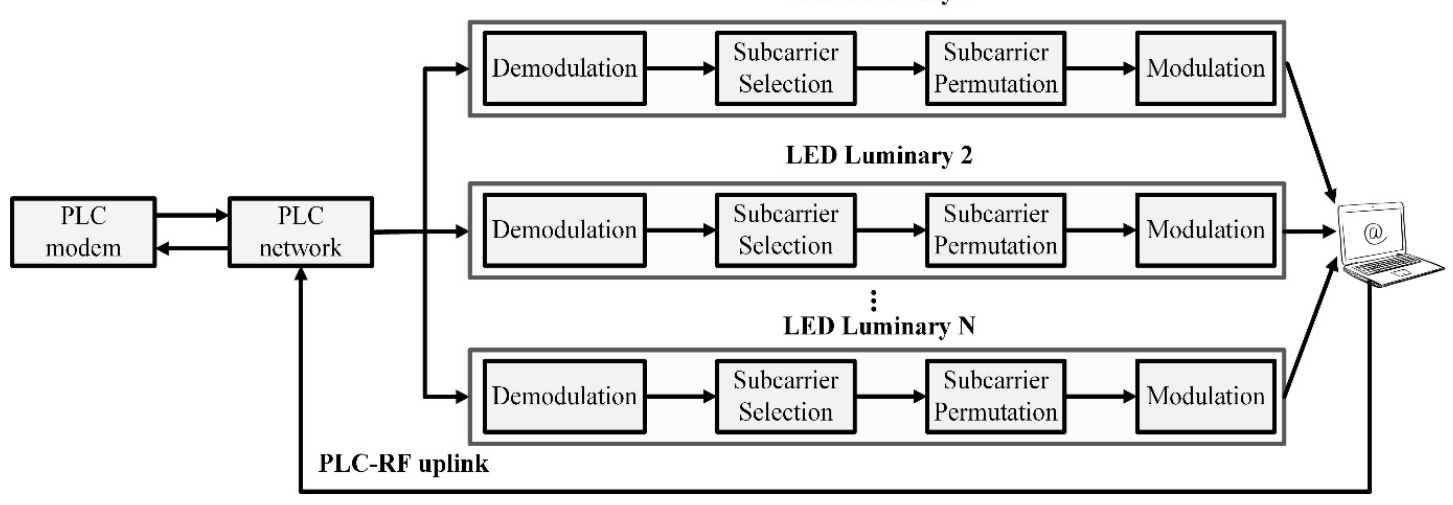

Figure 8. Block diagram of the hybrid IWOC and PLC for indoor multiuser downlink [14,23].

Specifically, in the above scheme, the basic mathematical model of the proposed system is to be submitted, specifically considering static continuous noise, periodic static continuous noise, and periodic pulse noise. In the visible light link, only the contribution of line of sight (LOS) component and the influence of stray light scatter noise and receiver preamplifier noise are considered. At the same time, the effect of multipath fading on the performance of the system is ignored since the detector size is much larger than the optical wavelength. Through the quantitative analysis results, the team further confirmed that the IWOC link can provide SNR up to $22 \mathrm{~dB}$ to meet the functional requirements of IWOC and basic lighting. Therefore, the overall performance of the integrated system is to a great extent affected by the PLC link.

Researchers also try to introduce OFDM and color shift keying (CSK) technologies into the IWOC channel, and study the behavior of these series channels under the condition of full link transmission [29]. Specifically, in the PLC link, researchers sought to apply the quadrature phase shift keying (QPSK) and OFDM modulation schemes. In IWOC link, CSK scheme is utilized to improve system performance. The researchers are determined to the mapping scheme design of OFDM modulation constellation map of PLC link to CSK constellation map in IWOC link.

Facing the communications scene between outdoor buildings, researchers in South Africa proposed the integrated scheme of PLC and IWOC to conduct targeted exploration. Similarly, specific system schemes introduce phase shift keying (PSK) and CSK modulation respectively in PLC and IWOC links [30]. The mapping method between PSK and CSK modulation symbols is given and verified by simulation and experiment. At the same time, the interference effect of solar rays on the red, green, and blue symbols of CSK is analyzed and emphasized. Based on the above research work, investigators further try a different modulation scheme combines to obtain the further improvement of the system performance. Specifically, spread frequency shift keying (S-FSK) is processed in PLC link, and an on off keying (OOK) modulation is tried in IWOC. The significant advantages of the scheme depended on its low cost and low complexity. Correspondingly, the disadvantage is the case that the transmission rate is extremely limited. Through the prototype experiment based on this scheme, the researchers measured the impact of sunlight exposure on the system performance at four different points in the day, and determined that sunlight irradiance is the main source of system noise [31,32]. 
The various applications of hybrid PLC and IWOC technologies have been studied in [1,33-36]. The first integrated scheme using OOK for data modulation is discussed in [35]. On this basis, OFDM technology was used in hybrid IWOC/PLC system to decrease inter-symbol interference [33]. In [34], an integrated PLC and IWOC communication system based on OFDM modulation was proposed to provide electronic medical services inside the hospital. For cascaded BPLC and IWOC communication systems, the performance of binary phase shift keying (BPSK) OFDM for subcarrier modulation is studied to reduce the impact of impulse noise and ISI. In [36], the opportunity of PLC and IWOC channel integration was investigated. Literatures $[37,38]$ analyzed the capacity of hybrid IWOC/PLC systems based on AF and DF. First, the source node passes the information to the relay node through the PLC link, and then the relay node amplifies [39] or decodes [20] the transmitted information, and finally forwards the information to the destination node through the IWOC link. A hybrid system of IWOC and RF is considered in the indoor environment [40] to improve the average throughput and interrupt throughput of each user. In [1], a cascaded IWOC/PLC system is considered, which is compatible to provide a variety of services with different service quality requirements.

\subsubsection{Hybrid Optical Wireless System in NOMA Mode}

Non-orthogonal multiple access (NOMA) is deemed to be another promising technology that can significantly improve spectral efficiency and connectivity. Different from orthogonal multiple access (OMA), multiple users in NOMA could transmit on the same frequency resources at the same time by superimposing the signals of multiple users in the air. At the receiving terminal, successive interference cancellation (SIC) could be applied to sequentially decode each individual signal from the received composite signal. It has been proven that NOMA could increase spectral efficiency and promote large-scale connectivity. Meanwhile, NOMA signaling can reduce the cost of communications systems to help provide low-latency services. In [41-43], researchers analyzed the signal-to-noise (SNR) about different OFDM modulation methods of PLC and IWOC system.

It is anticipated that the application of NOMA to the IWOC systems will further increase the system capacity and user connectivity [44,45]. In [45], it was shown that the spectrum efficiency of IWOC system with NOMA function is superior to that of IWOC system with conventional orthogonal frequency division multiple access (OFDMA). In [45], the authors analyze the performance of the coverage probability and the ergodic rate. The results show that using NOMA could enhance these performance metrics compared to OMA. Though there have been some pioneering works in the NOMA-enabled IWOC systems, these studies mainly focus on low order modulation schemes.

Furthermore, Lin and his researchers in [46] proposed a NOMA scheme based on VLC based on optical power domain to address the deficiencies of the traditional PD-NOMA scheme. The maximum drive current of the scheme is significantly reduced, and the gain bandwidth product of the drive circuit is also obviously reduced. Experimental results show that the OPD-NOMA scheme provides better bit error rate performance. The experiments are as shown in Figures 9 and 10. Where Figure 9 shows the experimental setup of the proposed IWOC-OPD-NOMA for two users [46]. Use arbitrary waveform generator (AWG, RIGOLDG5352) to convert digital OFDM signal to an analog format. Note that the AWG is also used as a driver amplifier. The detail block diagram is shown in Figure 10. In $T_{X}$, two 1.7 Mbaud-based band OFDM signals are mapped to QPSK and converted to an RF subcarrier whose frequency is $1.25 \mathrm{MHz}$. When the symbol rate is $1.7 \mathrm{~m}$, the maximum output peak voltage $(\mathrm{Vpp})$ is $20 \mathrm{~V}$. For the driver amplifier, the gain-bandwidth product is a constant [46]. As the symbol rate increases, the maximum output value decreases. The LEDs in the LED array are divided into two groups, and each group is assigned to a user. The OFDM electrical signals of different Vpp use the offset tee module to perform DC level shift before the IM of the grouped LEDs. The modulation bandwidth of the LED is $2.5 \mathrm{MHz}$ [46]. 


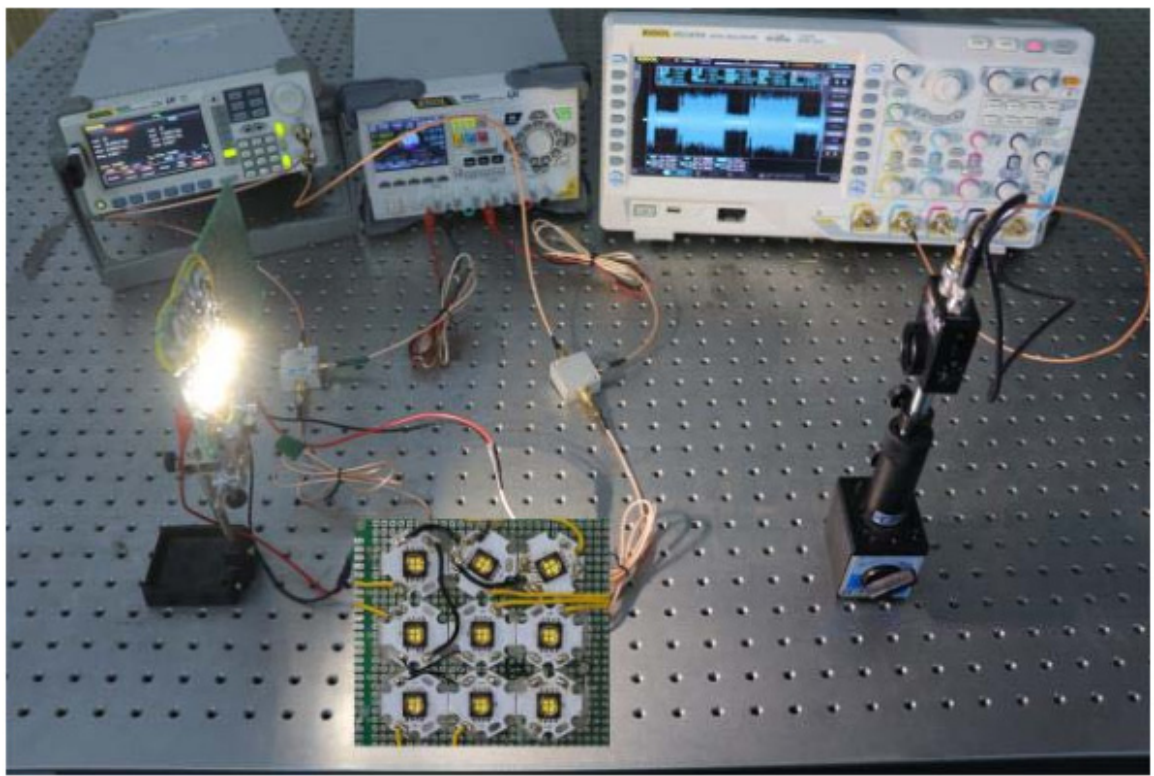

Figure 9. Experiment setup for IWOC-OPD-NOMA [46].

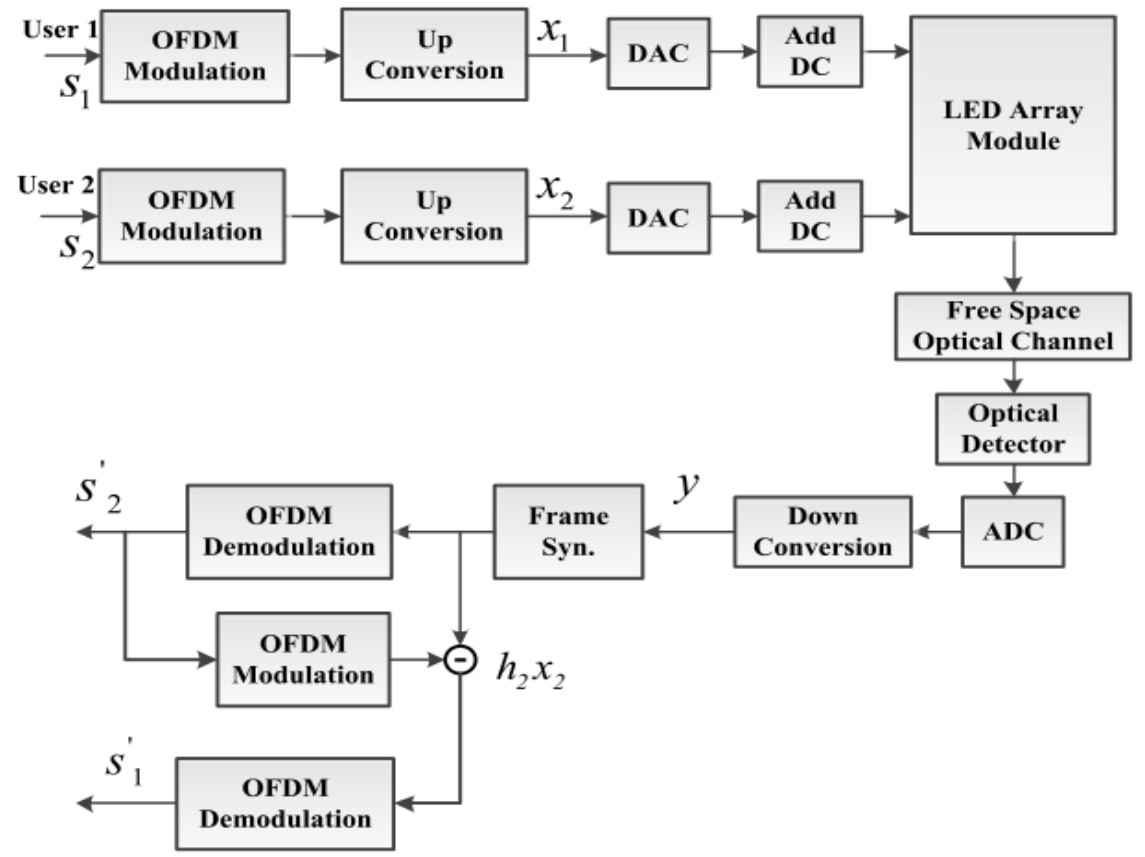

Figure 10. Block diagram for the downlink IWOC-OPD-NOMA [46].

In addition, the bit error ratio (BER) comparison between NOMA and OMA is shown in Figure 11. As can be seen, it could be used in the OMA low SNR regime [47]. On the contrary, the simulation results of NOMA show that compared with OMA, the BER is derived under the condition of low SNR regime more accurately. Furthermore, it should be noted that NOMA has a worse BER than OMA owing to the multi-user interference in NOMA.

Specifically, for the user that is decoded in NOMA first, the influence of this mutual interferences is more obvious. For the user that is decoded last in NOMA, if the SIC performance is good enough, its bit error rate performance is similar to that of OMA. In addition, for users that are decoded first in NOMA, when a higher modulation scheme is utilized, the BER gap between OMA and NOMA will 
increase. This is because under the same transmission SNR, the constellation of high-order modulation schemes is more susceptible to noise [47].

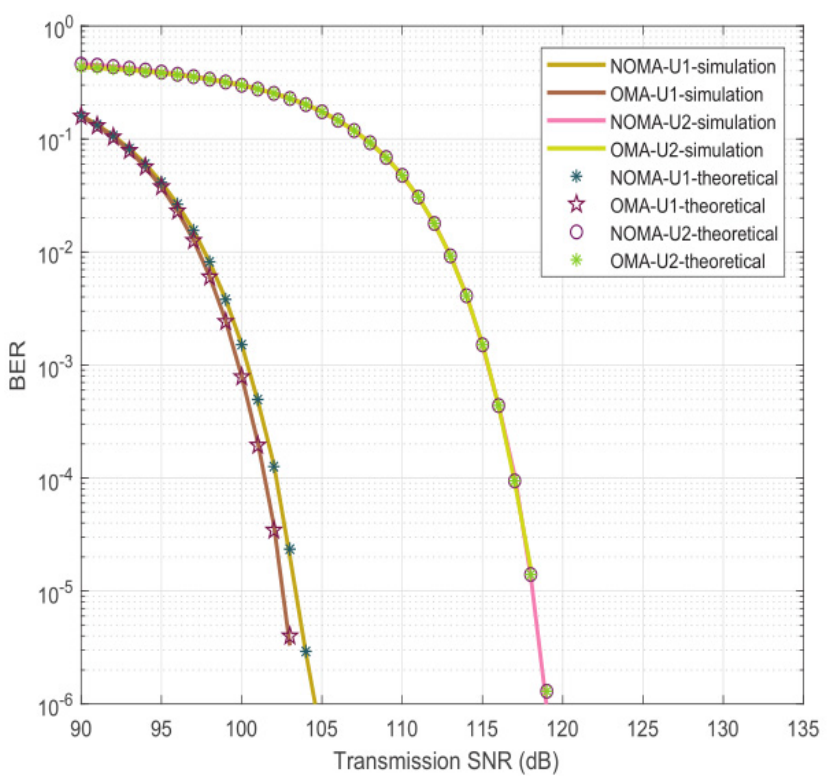

(a)

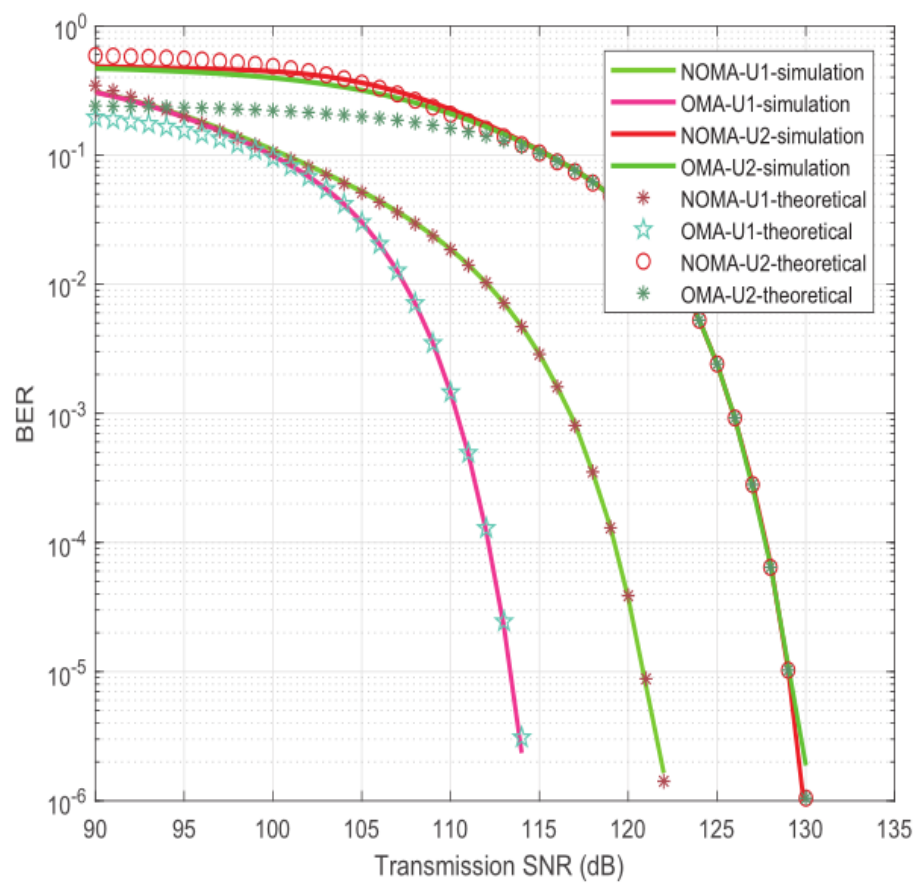

(b)

Figure 11. (a) The comparison of non-orthogonal multiple access (NOMA) with OMA in terms of BER with QPSK; (b) the comparison of NOMA with OMA in terms of bit error ratio (BER) with 16PSK [47].

In [48], cellular respiration has been applied to traffic transmission between devices in cellular networks and access point. In [49], a distributed algorithm based on pricing has been used, which regards the congestion degree of the base station (BS) and the transmission environment of mobile terminals as parameters of load balancing in heterogeneous networks. 
On the bases of line voltage, the lines in the transmission network can be divided into high voltage, medium voltage, and low voltage in [50-53]. In general, the power system includes generation, transmission, distribution, and consumption [51]. HV generates the power and transmits it. Then MV and LV regions distribute the power, and finally LV completes the consumption [51]. Typically, the range of high, medium, and low pressures varies depending on the region, industry, and so on. Generally speaking, the standard voltage classes for HV are between $110 \mathrm{kV}$ and $380 \mathrm{kV}$, such as $132 \mathrm{kV}$ and $220 \mathrm{kV}$, the standard voltage classes for MV are from $10 \mathrm{kV}$ to $33 \mathrm{kV}$, like $13.2 \mathrm{kV}$ and $33 \mathrm{kV}$, which are mentioned in [54], and the LV classes are below $1 \mathrm{kV}$, generally $0.24 / 0.4 \mathrm{kV}$ [52-54]. Considering the rapid development of power grid in large and super-large cities in recent years, the HV is going to $500 \mathrm{kV}$.

Distinct from the low-voltage power line studies above, researchers from Penn State University discussed the transmission characteristics of medium and low piezoelectric transmission media and IWOC system [50]. According to [50], both medium-voltage and low-voltage power networks can provide very high transmission capacity that cannot be achieved by any other cable network except optical fiber, and the reflection caused by the impedance mismatch in the above networks will worsen the performance of the system. At the same time, the research results show that the PLC subsystem has the possibility to realize $1 \mathrm{Gbps}$ transmission rate through the reasonable network design for instance impedance matching. In the IWOC subsystem, the different influences of multipath propagation on high-speed data transmission are considered. LEDs are commonly used in indoor settings as transmitters of OWC. Therefore, in [50], the authors consider the IWOC model, and conclude that distortion are related to room size and system configuration, and the above distortion can be minimized through judicious design. This work theoretically preliminarily proves the technical potential of medium and low voltage PLC and IWOC integrated systems to provide high-speed transmission.

In order to verify the actual performance of smart power OWC, research teams at domestic and abroad actively carry out the construction and test of power wireless optical system [55-57]. Furthermore, to better meet the need of hot scenes such as hospitals, shopping malls, and gymnasiums, researchers from Tsinghua University are committed to applying integrated PLC and IWOC technologies to indoor broadband data broadcasting. The proposed system significantly reduces the complexity of IWOC network protocol and the degree of infrastructure transformation.

Specifically, the researchers gave an experimental demonstration of LED two-light network and evaluated the performance of the proposed scheme [15], as shown in Figure 12. The high-definition television (HDTV) program is directed to the hybrid system through the time domain OFDM technology. The measurement shows that the bandwidth of the system is $8 \mathrm{MHz}$, specifically distributed between $2 \mathrm{MHz}$ and $10 \mathrm{MHz}$.

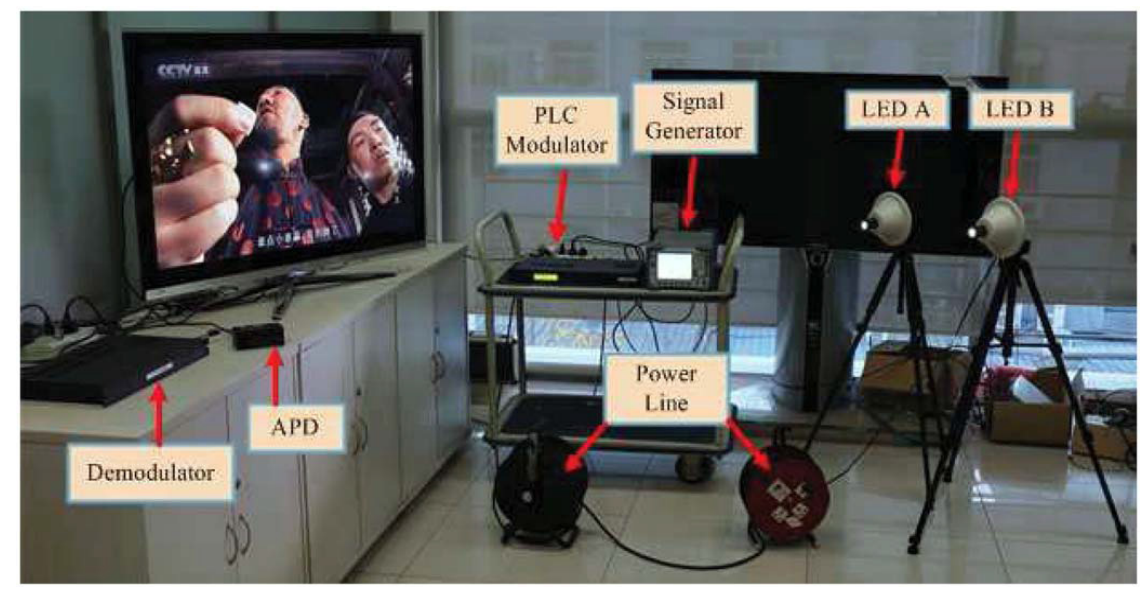

Figure 12. Demonstration setup of the PLC and IWOC broadcasting system in the laboratorial environment [15]. 
Under the condition that the visible light path length is extended to $8 \mathrm{~m}$ and the indoor interference lighting source is normally turned on, and the proposed hybrid system can provide a transmission performance of about $48 \mathrm{Mbps}$. Since the typical ceiling height is $3 \mathrm{~m}$, the receiver consisting of an avalanche diode is placed in an overlapped area covering both lights at a distance of $3 \mathrm{~m}$. To evaluate the system performance under different display configurations, the researchers kept the transmitting signal power of left LED constant, and evaluated the system performance changes under different multipath propagation conditions by adjusting the power line length connected to right LED and the transmitting power level of right LED, as shown in Figure 12 [15]. The result shows that the modulation error ratio (MER) is proportional to the power line length and inversely to the power of right LED. The above phenomenon can be explained as: when the secondary path increases or the power level decreases, the interference level will be correspondingly reduced. Therefore, attention must be paid to the profound influence of inter modulation interference on the performance of the receiving end.

To further illustrate the various modules in Figure 12, Song et al. from Tsinghua University illustrated the different hardware devices of PLC and IWOC broadcasting system in the laboratorial environment in Figure 13, including LED temp, PLC to IWOC module, PLC modulator, and demodulator in receiver [15].

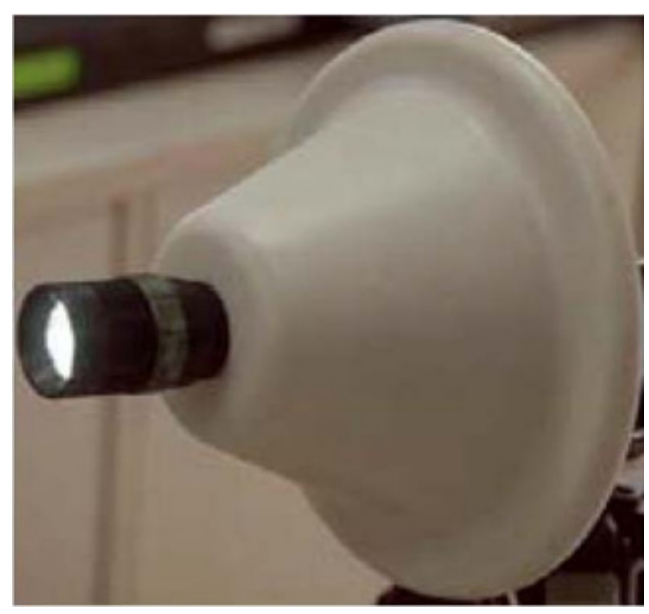

(a)

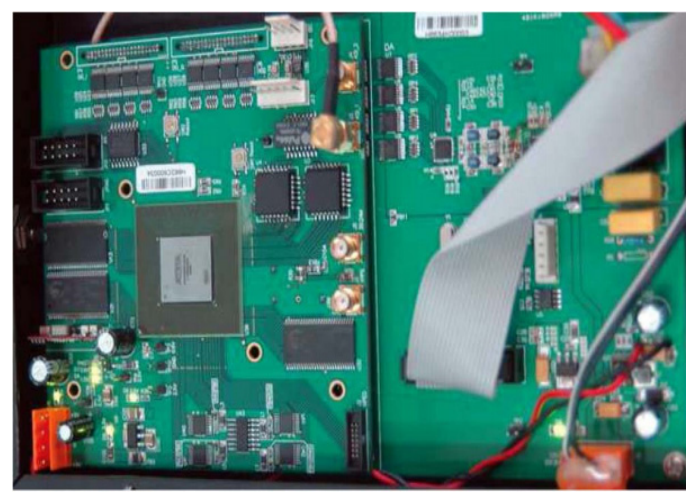

(c)

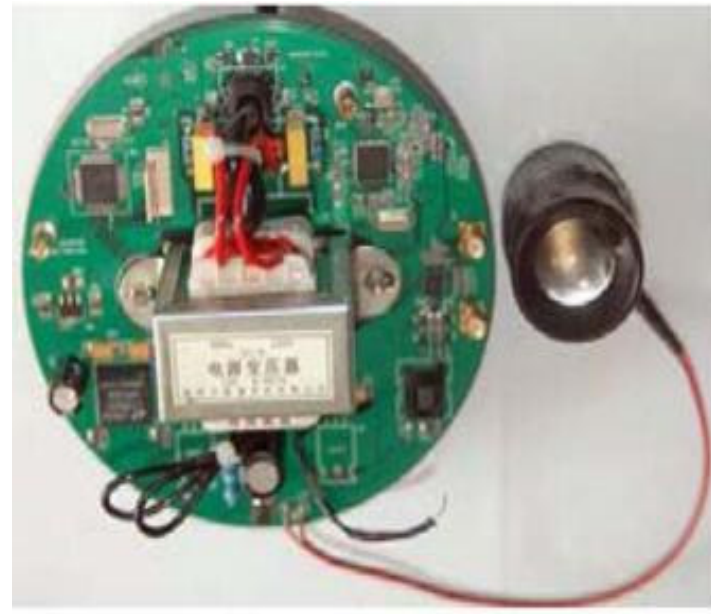

(b)

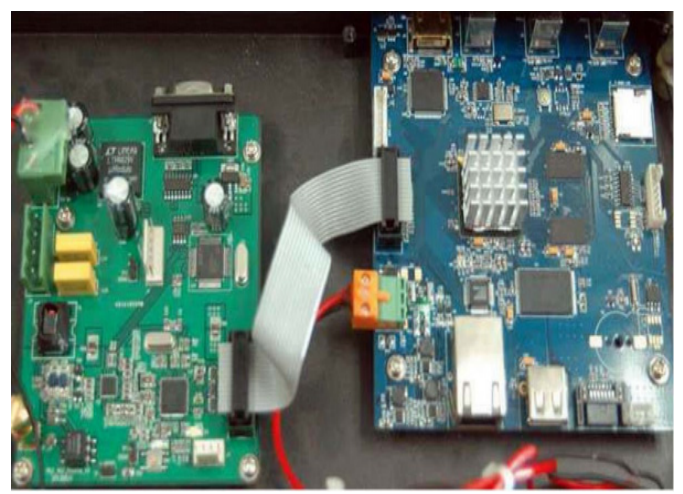

(d)

Figure 13. The hardware devices of PLC and IWOC broadcasting system in the laboratorial environment: (a) LED temp [15]; (b) PLC to IWOC module [15]; (c) PLC modulator [15]; (d) Demodulator [15].

In addition, they proposed extending the above system to underground tunnel sites in the next year, as shown in Figure 14. They implemented the IWOC-PLC integrated duplex communications network, and equipment mainly consisting of the LED fixture with DF module and IWOC-PLC coupler, 
overview of IWOC-PLC access network [17], duplex IWOC handheld terminal, and $\mathrm{R}_{X} \& \mathrm{~T}_{\mathrm{X}}$ modules in handheld terminals [17]. The display system is based on decoding and forwarding technology to realize duplex transmission, so as to build a symmetric duplex broadband access network to support diversified duplex high-speed communication services. In the underground tunnel scene, a plurality of light emitting diodes are used to realize the lighting function, and they are connected by power lines. In the experimental prototype, the length of the power line exceeds $100 \mathrm{~m}$.

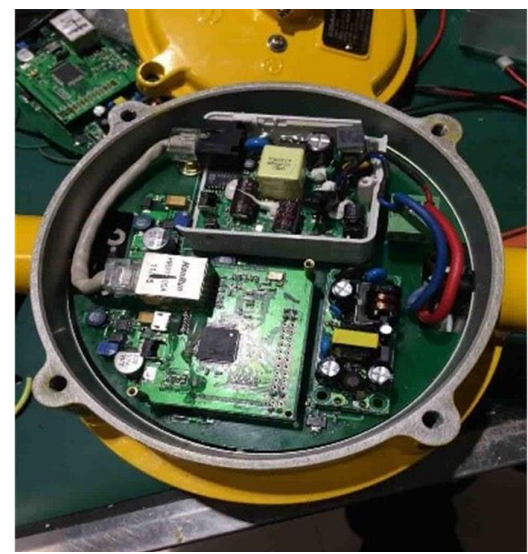

(a)

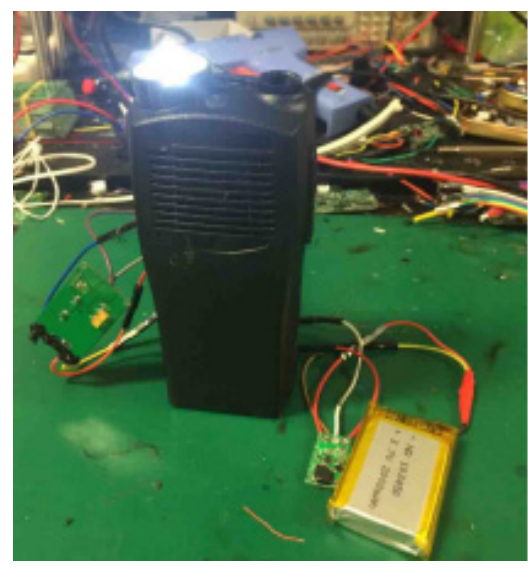

(c)

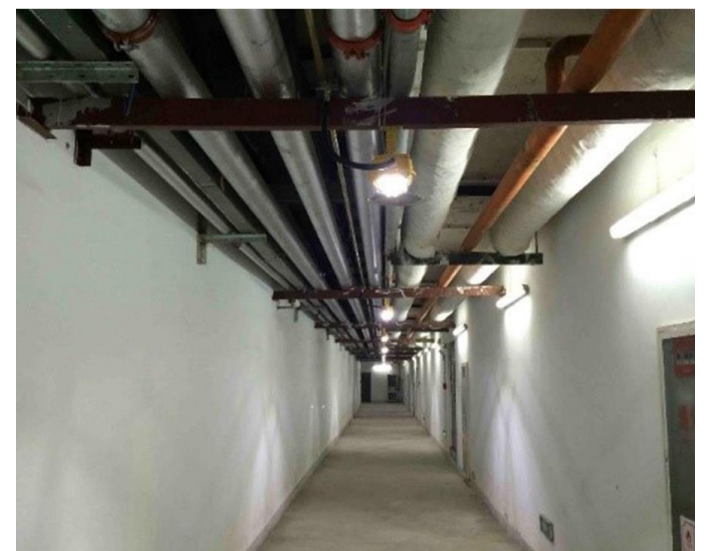

(b)

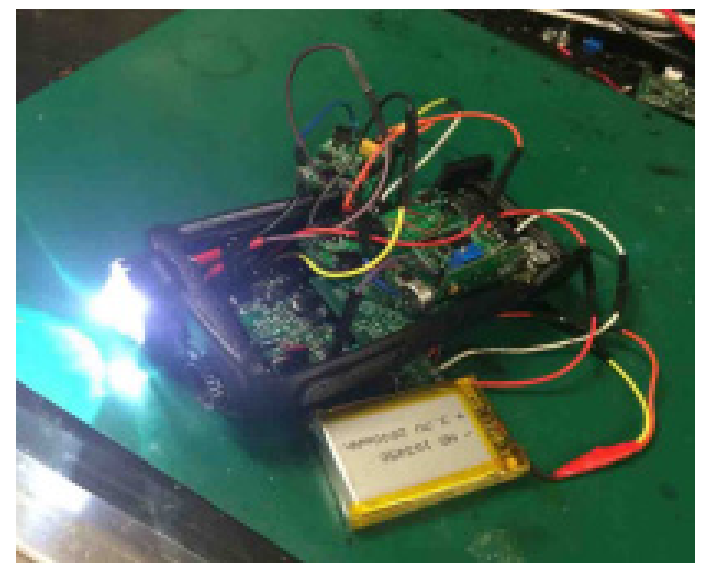

(d)

Figure 14. The IWOC-PLC integrated duplex communications network: (a) The LED fixture with DF module and IWOC-PLC coupler [17]; (b) overview of IWOC-PLC access network [17]; (c) duplex IWOC handheld terminal [17]; (d) $R_{X} \& T_{X}$ modules in handheld terminals [17].

In DF module, PLC and IWOC coupler, uplink photodetector is encapsulated into LED device. In addition, the handheld terminal is embedded in the IWOC receiving and sending modules. Experimental test results show that the distance between LED light source and receiving terminal is $3 \mathrm{~m}$ under the condition, the above system can support voice, video, and duplex broadband communication up to $5 \mathrm{Mbps}$. As long as 3 days of field test, BER performance can be maintained at $10^{-5}$. The data rate of the system can be further extended to $30 \mathrm{Mbps}$. In the multi-user scenario, efficient and cost-effective non-interference communications are realized between different users. For the application of power tunnel, the research team of state grid also proposed the design of IWOC and PLC protocol for power tunnel inspection and the patrol scheme of LiDAR-based navigation UAV, providing new technical options for the enhancement of power wireless optical patrol technology $[55,56]$.

In addition, the research team from the Institute of Semiconductors of the Chinese Academy of Sciences focused on smart home scenarios and carried out home network design and a prototype 
implementation are based on IWOC and PLC. The proposed core equipment of the home network system mainly includes home gateway, white LED driver and network appliance. Among them, the home gateway assumes the function of connecting with external network or external personal consumption terminal. The controlled terminal in the home network is called network appliance. Each communications unit is interconnected by PLC bus and gateway to realize independent two-way communications. The test results of this system show that 22,953 instructions are sent through the network transmitter for testing, and the network receiver can correctly receive 22,829 instructions, and the correct transmission rate of system instructions is $99.46 \%$, meeting the basic requirements of actual use [50].

\subsection{Integrated Transmission Schemes of Power Line and Indoor Wireless Optical Communications}

PLC and RF integration into wireless relay systems has been proposed for many applications, including indoor, outdoor, and smart grids [29,58-62]. In [63], a cascaded IWOC and outdoor WOC communications system is studied and DF relay is established. The capacity of the system is studied, and the results show that the hybrid system has high data transmission efficiency. Some different integrated scheme of power line and IWOC is shown in Figure 15. DF relay hybrid IWOC-PLC scheme, hybrid IWOC-PLC scheme, multichannel hybrid IWOC-PLC system and full duplex DF IWOC-PLC scheme are proposed $[15-17,19]$. Where the PLC represents for the IWOC.

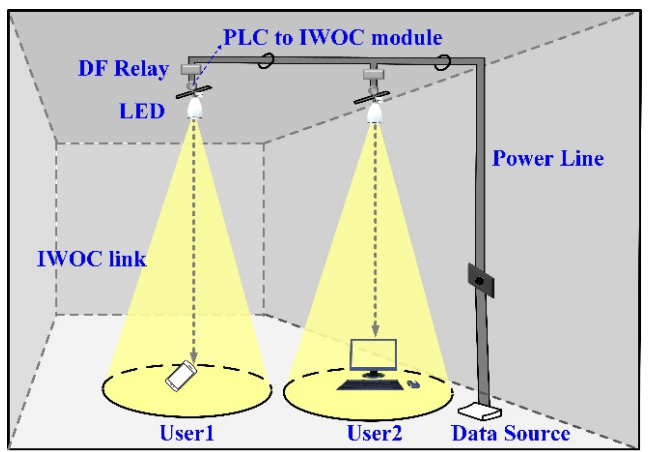

(a)

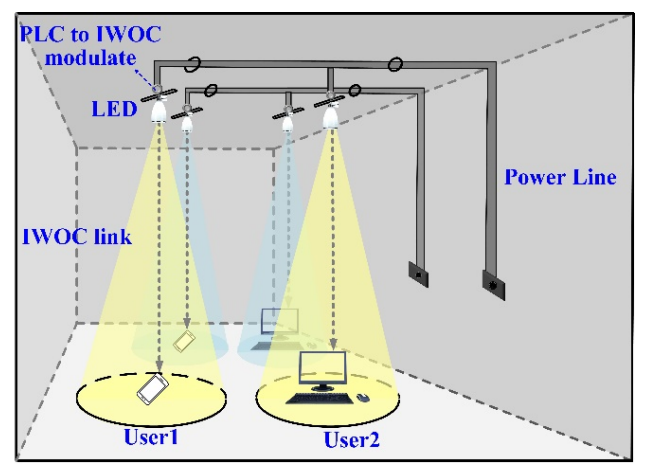

(c)

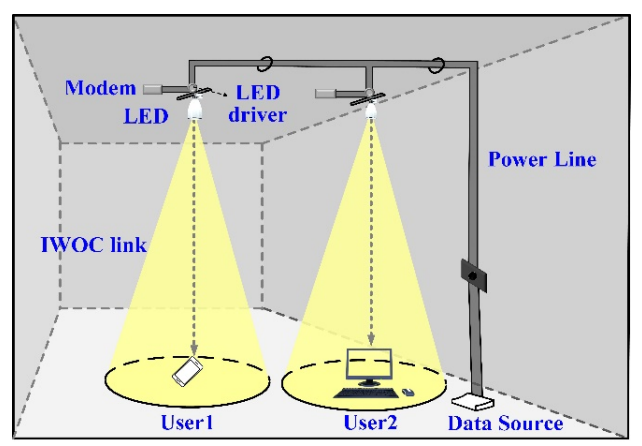

(b)

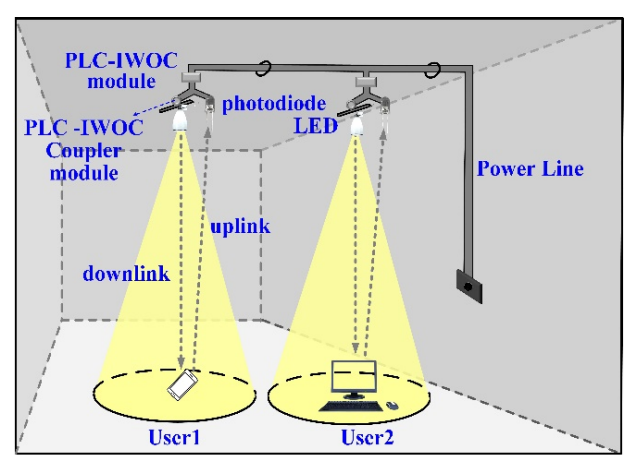

(d)

Figure 15. The different schemes of IWOC-PLC systems: (a) DF relay hybrid IWOC-PLC scheme [15], (b) hybrid IWOC-PLC scheme [15,16,19]; (c) multichannel hybrid IWOC-PLC system [15]; (d) full duplex DF IWOC-PLC scheme [17].

Indoor multi-user downlink of hybrid circuit and IWOC must face the limited transmission bandwidth of power line and visible integrated channel. In order to improve the transmission spectrum efficiency of the above hybrid system, more and more researchers have proposed multi-carrier system 
scheme to better cope with the frequency selectivity of the channel $[27,28,35,41,64,65]$, as shown in Figure 16. In this paper, the IWOC in the module in Figure 16 is represented by IWOC.

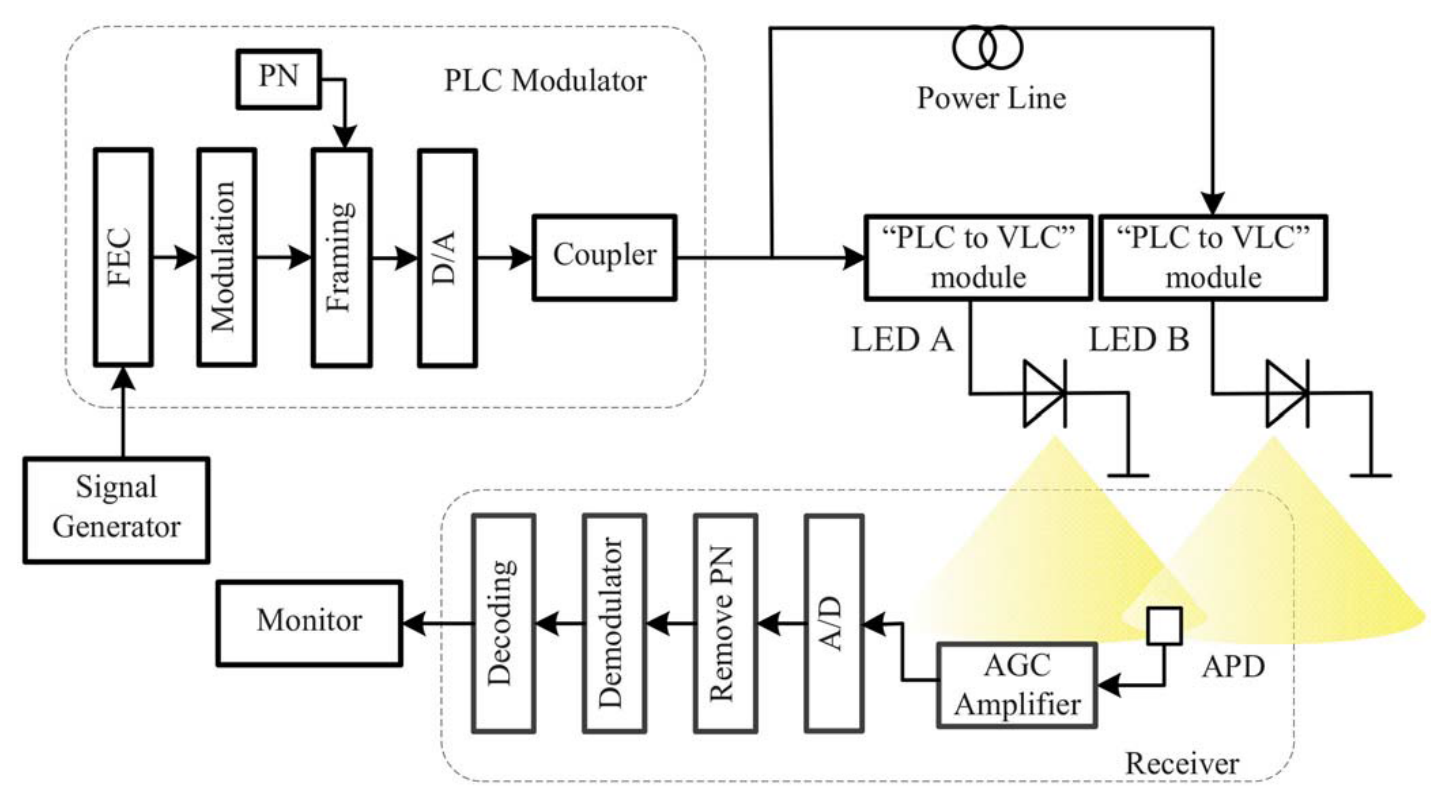

Figure 16. Block diagram of the integrated PLC and IWOC multicarrier system [15].

On the basis of the above work, researchers developed a PLC-oriented visible light positioning system and presented a laboratory prototype demonstration [15], as shown in Figure 17. The above prototype leverages an Android application to achieve a few KBPS level of low speed links. This work will help speed up the commercialization of such systems and prototypes. Such a power wireless optical positioning system can make full use of the orientation of LED beam and PLC return network to provide users with customized services based on user location, including indoor navigation, device tracking, and equipment management [15,19]. In addition, existing indoor monitoring or sensing device can also realize the power supply and communications function of the above system at the same time, so as to timely transmit the monitoring or sensing data to the indoor wireless terminal. In $[1,12,15,17,33,36,43,50,66]$, these studies have shown that the cooperative relay aided PLC-IWOC system has not been thoroughly analyzed according to various statistical parameters. In [12], the transmission power optimization problem of hybrid IWOC/PLC/RF communication system is discussed.

It must be pointed out that the research and implementation of power wireless optical positioning technology is still in its infancy. Some of the more complex and potentially high-precision visible location technologies have not been fully explored in this field. These algorithms usually need to measure the reception intensity information, arrival angle information, arrival time information, or arrival time difference information of light signals from different LED light sources. Based on such information and the location information of the light source, the spatial location of the mobile terminal can be reversely estimated [6]. The existing research work often stops at the positioning algorithm itself, without fully considering the actual factors such as interconnection relationship, physical characteristics, and synchronous performance of the power line network connected with the LED light source to the potential influence on the performance of power wireless optical positioning technology. Therefore, it is necessary to carry out targeted performance improvement work on the basis of comprehensive consideration of the above factors, so as to make the high-precision power wireless optical positioning technology from theoretical analysis to practical application. 


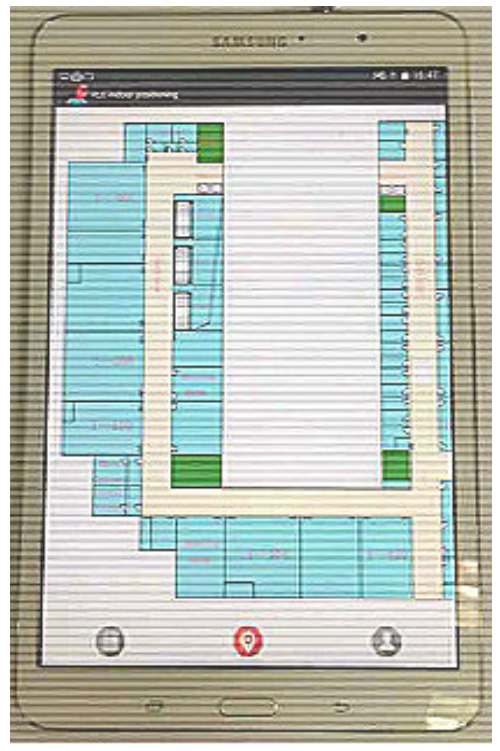

(a)

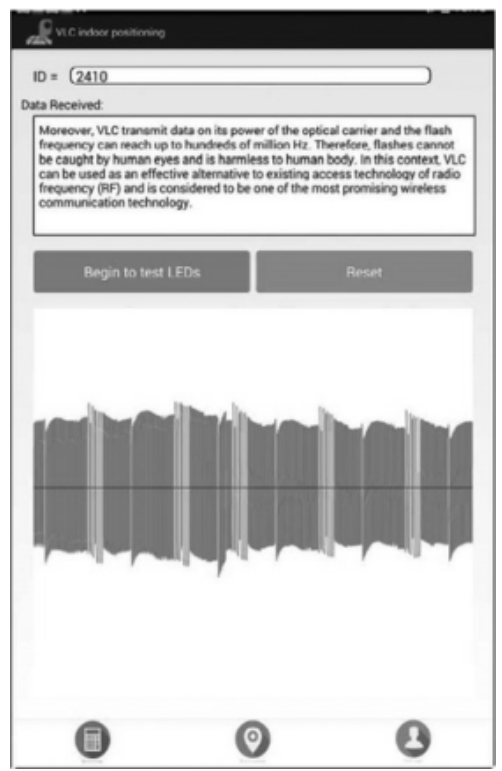

(c)
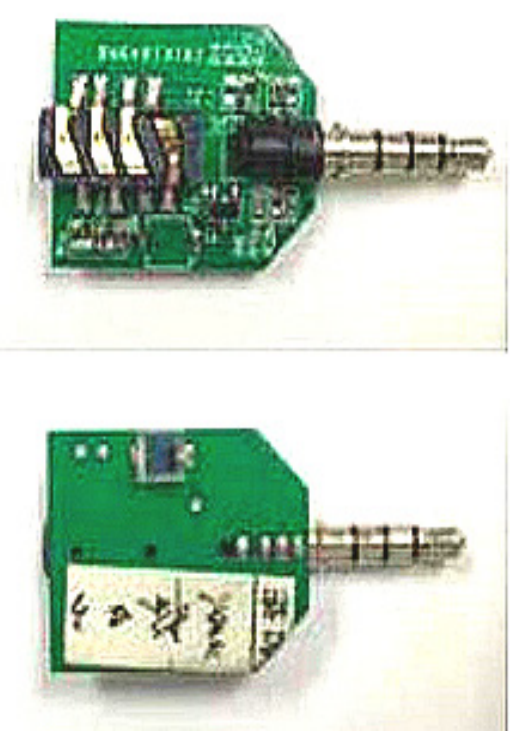

(b)

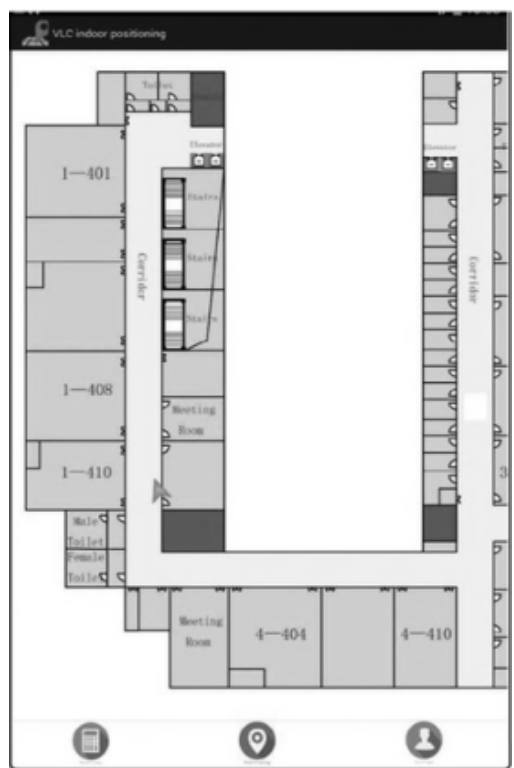

(d)

Figure 17. Demo and Android APP for indoor localization based on IWOC: (a) demo [12]; (b) receiver (back and front) [12]; (c) signal detection in the Android APP [16]; (d) map localization in the Android APP [19].

Paper [37] studies the link of relaying cascade PLC and IWOC with amplify and forward (AF) relay, based on that AF relay has not been considered to connect PLC and IWOC links before. In the first stage, the source transmits the information to the relay through the PLC link. In the second stage, AF relay amplifies the received signal and forwards it to the target node via IWOC channel. Setting the total capacity of a hybrid IWOC/PLC system provides the opportunity to examine the performance of such a system and the effect of different system parameters such as relay position and relay distance to the target. The accuracy of the results is verified by Monte Carlo simulation. 
For comparison, the performance of the hybrid system is compared with that of the hybrid/PLC, in which a linear combination is used to maximize the overall capacity of the system. In [67], the coordination between the light sources and how to improve the performance of the IWOC system are studied. The author verifies that the implementation of decode and forward (DF) relay has better performance than AF relay, as shown in Figure 18. To further improve the performance of PLC and IWOC cooperative system, researchers introduced relay technology into such IWOC indoor broadcast communication system [37,38,68-70]. In [69], PLC link is connected with IWOC link through DF relay and becomes the backbone of IWOC link. This paper investigates both AF and DF protocols for IWOC-based uplink transmissions. In traditional cellular mobile networks, relay technology can significantly improve network throughput and extend coverage. Inspired by this, PLC and IWOC system can also be regarded as relay-assisted dual-hop communication systems. At the signal transmission level, there are two main relay technologies to choose from, namely decoding and forwarding and amplifying and forwarding.

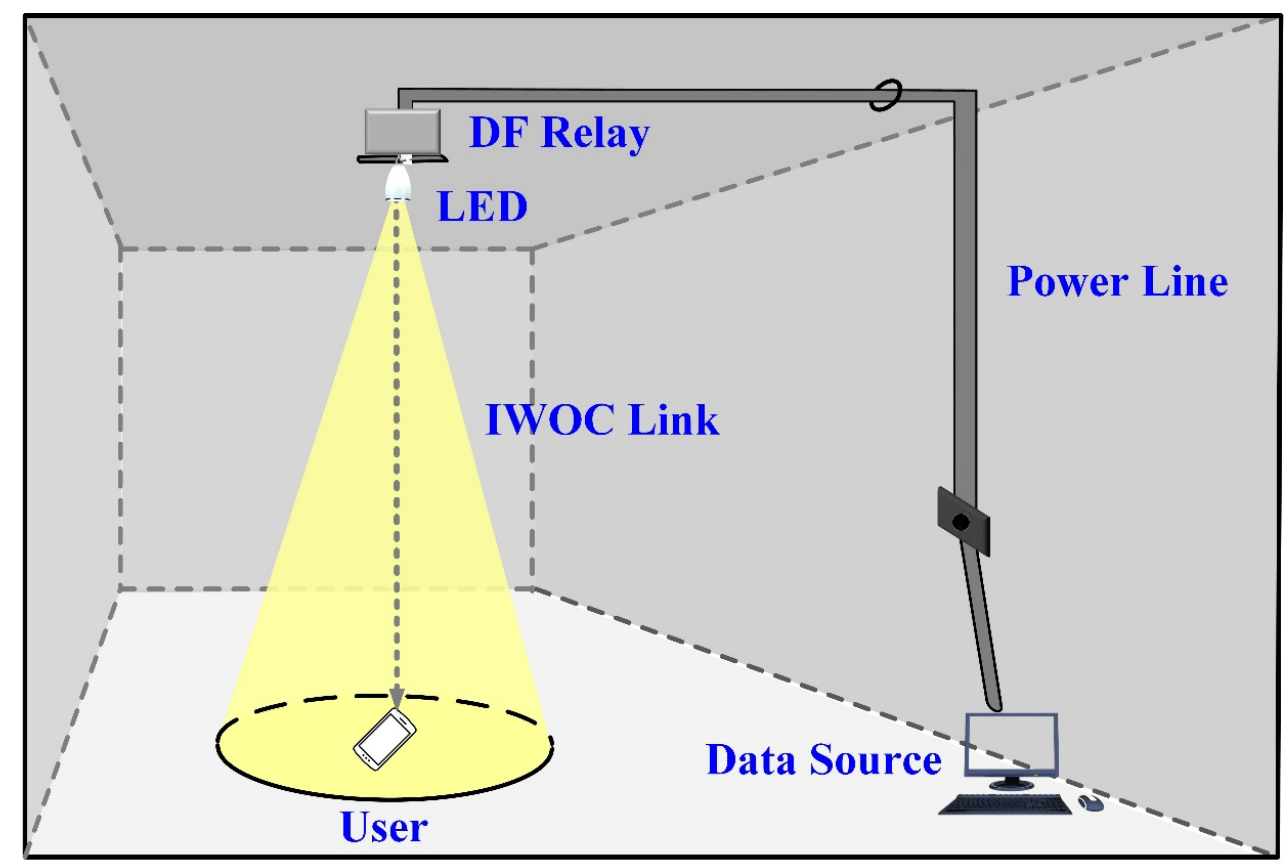

Figure 18. System model for the DF relaying hybrid IWOC/PLC network for indoor communication applications [67].

In the decoding, forwarding, and relay scheme, PLC signals need to be demodulated and decoded for PLC transmission before being loaded into LED light source, and then re-coded and re-modulated for IWOC transmission [38]. At the same time, in the amplification code forwarding and relay scheme, the analog PLC signals superimposed with noise components are directly amplified and sent when loaded onto the LED light source, without any coding operation [66]. Specifically, The PLC subsystem assumes part of the return link of IWOC link, which is susceptible to log-normal distribution fading and additive noise. The former method can completely eliminate the cumulative effect of the above noise by DF, but the implementation complexity is relatively high. Although the latter scheme cannot eliminate the influence of noise, it does not involve complex decoding and encoding operation, and the implementation complexity is very low, which is beneficial to significantly reduce the implementation cost of the system. At present, international research teams have proposed to provide services for users through multiple IWOC access points in IWOC link, as shown in Figure 18. The simulation indicates that the overall characteristics of system depend on the random distribution of the multi-user in the end terminal. By selecting the diversity combination technology, the appropriate access point can be 
selected for the user, and then the maximum instantaneous SNR can be provided for the user. On the basis of a single relay node, the performance gain can be further introduced into the system.

For the sake of enhance the throughput performance of integrated PLC and IWOC systems, more and more researchers are engaged in related research work. Specifically, the research team from the University of Southampton proposed a NOMA joint power allocation strategy for PLC and IWOC networks [13]. The results show that NOMA technology has a higher throughput performance than OMA in terms of multiple minimum rate requirements and user density. The main defect of IWOC technology is the performance degradation under LOS path missing. RF communication, with its higher penetration capability, can overcome the above deficiencies. In fact, the fusion of PLC technology and wireless network has been proposed in many application scenarios. This includes integrating PLC technology into the wireless relay system in smart grid applications, so as to realize long-distance data transmission [66]. Based on the above considerations, researchers from Texas A\&M University in Qatar proposed a hybrid PLC, IWOC, and RF communications system [66,71], as shown in Figure 19 [12]. To satisfy the required quality of service (QoS), researchers constructed and analyzed the overall transmission power minimization problem.

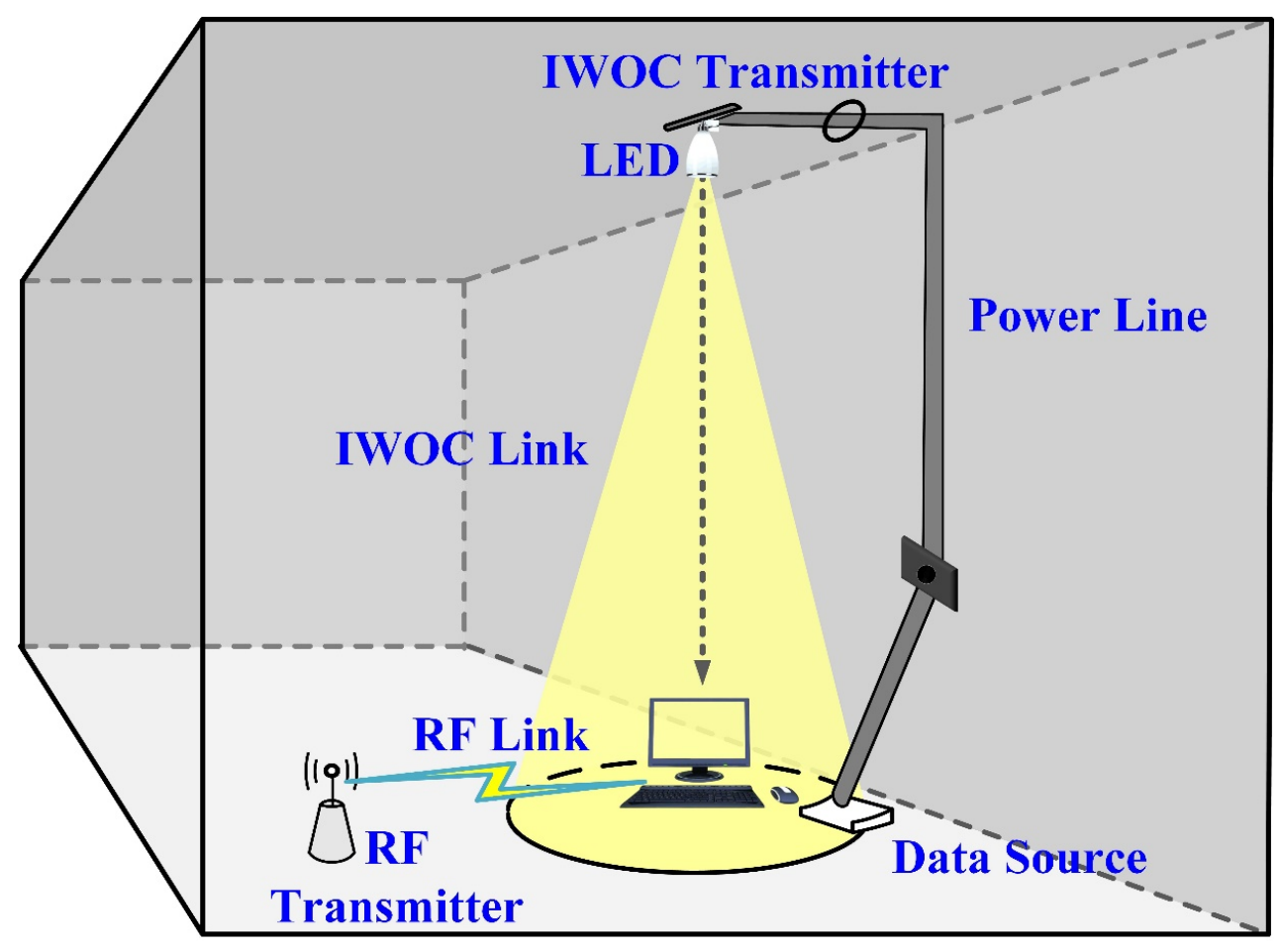

Figure 19. Hybrid PLC/IWOC/RF communication system model [12].

To coordinate the collaboration between RF networks and hybrid PLC and IWOC networks, the team discusses resource allocation between two parallel links, namely RF links and hybrid PLC and IWOC links. The proposed system explores multiple wireless interfaces to accumulate the parallel data streams received. The results show that the above optimization problems can be constructed as convex optimization problems and can be solved by means of computational efficient behavior. The quantization results show that the actual system parameters such as PLC channel model and RF signal availability have a significant influence on network performance. Therefore, as a promising architecture, the parallel system of RF, PLC, and IWOC could enhance the rate and reliability of indoor wireless network.

In [72], a new hybrid outdoor WOC/RF-IWOC system is proposed, which chooses to combine the selection between outdoor WOC and RF modules to obtain a single noise with a higher SNR. At the relay terminal, the DF protocol is adopted for the signals with high SNR, and then the signals 
are forwarded to the multi-user through the IWOC link. In [59], a hybrid PLC/IWOC/RF future for the wired backhaul system based on optical fibers has been proposed to improve the realized SRC. Meanwhile, PLC-IWOC system also provides a lot of advantages, such as good security, economical and easy to deploy, good transmission efficiency, high capacity, and high data rate $[20,73]$. Thus, these systems could be a potential candidate to provide ubiquitous connectivity for Internet of Things (IoT) devices.

In these three subsections, we consider the channel characteristics of PLC and IWOC system, the modulation of integrated PLC-IWOC system, and different transmission schemes. Table 2 compares the different researches between PLC and IWOC system in these years.

Table 2. Comparison of different researches between PLC and IWOC system.

\begin{tabular}{|c|c|c|c|c|c|c|}
\hline Years & System Types & Key Techniques & $\begin{array}{l}\text { Consideration } \\
\text { Factors }\end{array}$ & Distance & Data & Ref \\
\hline 2015 & $\begin{array}{l}\text { Integrated } \\
\text { PLC/IWOC }\end{array}$ & AF relay & Multi-path effects & $3 \mathrm{~m}$ & $\begin{array}{l}48 \mathrm{Mbps} \\
\text { (Data rate) }\end{array}$ & [12] \\
\hline 2015 & $\begin{array}{c}\text { Hybrid } \\
\text { PLC/IWOC }\end{array}$ & $\begin{array}{c}\text { OFDM } \\
\text { modulation }\end{array}$ & $\begin{array}{l}\text { Satisfy the practical } \\
\text { application }\end{array}$ & $8 \mathrm{~m}$ & $\begin{array}{c}48 \mathrm{Mbps} \\
\text { (Data rate) }\end{array}$ & [16] \\
\hline 2015 & $\begin{array}{l}\text { Integrated } \\
\text { PLC/IWOC }\end{array}$ & $\begin{array}{l}\text { DF Relay and } \\
\text { BDM } \\
\text { modulation }\end{array}$ & $\begin{array}{l}\text { Multi-path effects and } \\
\text { attenuations }\end{array}$ & $2.8-3 \mathrm{~m}$ & $\begin{array}{c}0.4 \text { bit/symbol }\left(\mathrm{BDM}^{1}\right) \\
0.13 \mathrm{bit}^{1} / \mathrm{symbol}^{2} \\
\left(\mathrm{TDM}^{2}\right) \\
(\text { Data rate })\end{array}$ & [18] \\
\hline 2015 & $\begin{array}{l}\text { Cascaded } \\
\text { PLC/IWOC }\end{array}$ & $\begin{array}{l}\text { OFDM and FSK } \\
\text { modulation }\end{array}$ & Impulsive noises & $2.8-3 \mathrm{~m}$ & $\begin{array}{c}10^{5} \\
\text { (Data rate) }\end{array}$ & [62] \\
\hline 2016 & $\begin{array}{c}\text { PLC } \\
\text { backhauling and } \\
\text { Hybrid } \\
\text { IWOC/RF }\end{array}$ & OFDM & $\begin{array}{l}\text { Multi-user utility } \\
\text { function }\end{array}$ & $2.8-3 \mathrm{~m}$ & $\begin{array}{l}10 \text { Mbps (VLC) } \\
5 \text { Mbps (RF) } \\
\text { (Data rate) }\end{array}$ & [1] \\
\hline 2016 & $\begin{array}{l}\text { Hybrid } \\
\text { PLC/IWOC }\end{array}$ & $\begin{array}{l}\text { Duplex DF } \\
\text { underlying }\end{array}$ & Intercell interference & Over3 m & $\begin{array}{l}5-30 \mathrm{Mbps} \\
\text { (Data rate) }\end{array}$ & [14] \\
\hline 2018 & $\begin{array}{c}\text { Hybrid } \\
\text { PLC/IWOC/RF }\end{array}$ & $\begin{array}{l}\text { OFDM } \\
\text { modulation }\end{array}$ & Power allocation & $2.8-3 \mathrm{~m}$ & $\begin{array}{l}18.5 \mathrm{Mbps} \\
\text { (Data rate) }\end{array}$ & [9] \\
\hline 2018 & $\begin{array}{l}\text { Hybrid } \\
\text { PLC/IWOC }\end{array}$ & AF Relay & $\begin{array}{l}\text { Relay location and } \\
\text { relay-to-destination } \\
\text { distance }\end{array}$ & $3.15 \mathrm{~m}$ & $\begin{array}{c}0.04 \mathrm{bit} / \mathrm{s} / \mathrm{Hz} \\
\text { (Average capacity) }\end{array}$ & [36] \\
\hline 2018 & $\begin{array}{l}\text { Integrated } \\
\text { PLC/IWOC }\end{array}$ & DF Relay & $\begin{array}{l}\text { User's location and } \\
\text { transmit power }\end{array}$ & $2.75 \mathrm{~m}$ & $\begin{array}{c}0.04 \mathrm{bit} / \mathrm{s} / \mathrm{Hz} \\
\text { (Average capacity) }\end{array}$ & [60] \\
\hline
\end{tabular}

${ }^{1}$ BDM represents for bit division multiplexing. ${ }^{2}$ TDM stands for time-division multiplexing.

\subsection{Wireless Optical Sensor Monitoring of Power Lines}

High-speed communication on power industry allows for real-time monitoring of network. This allows to anticipate possible events, process those events more rapidly, and improve the quality of service to customers. Recently, communication infrastructures using broadband power lines, Wi-Fi, and optical fibers have been designed for power distribution network management services.

The authors in [74] compared the speed performances of each communication. Experimental results indicated that optics fiber is the fastest communication network, and could provide a bidirectional delay of $3 \mathrm{~ms}$. A significant factor to consider in the communications system is the costs of infrastructure investment. Sometimes slower solutions, for instance, PLC may be more appropriate for specific applications or when a large number of measuring devices must be installed in a distribution system. PLC is an example of a common and cost-effective means of communication for smart grid monitoring since it allows the power lines to be used for power and communication, thereby eliminating the need for special cables for transmission control and data signals. In reference [75,76], different overhead communication PLC lines were compared for several applications of power distribution networks. 
Researchers in Brazil introduced wireless optical technology into the wireless transmission of sensor signals on high-voltage power lines and carried out a series of work [77-79]. Optical fiber sensors include two types: distributed sensors or punctual sensors. The latter could be connected in a signal fiber, or they could be used in lots of different fibers which are connected in a single detection point one by one, for instance using a distributor [80]. Specifically, the current sensor transmits the sensor signal to the remote unit fixed on the high-voltage line. According to [77], the experiment applies a distributed feedback laser wavelength of $1530 \mathrm{~nm}$. Aerial cables are used to connect the remote room to the optical power phase conductor (OPPC), which also use single-mode (SM) fibers. Then multi-mode (MM) fibers are used to link to the collimator A and collimator B respectively. The FSO device is connected to the far end of the OPPC cable. Then the optical signal is transmitted from collimator A to the low-potential collimator B via FSO device. The optical signal is measured with a power meter, and data acquisition (DAQ) board collects data recorded by the computer. The block diagram of the optical wireless outfield monitoring device system is shown in Figure 20. Next, the optical signal carries the sensing information to the control unit through the MM fiber.

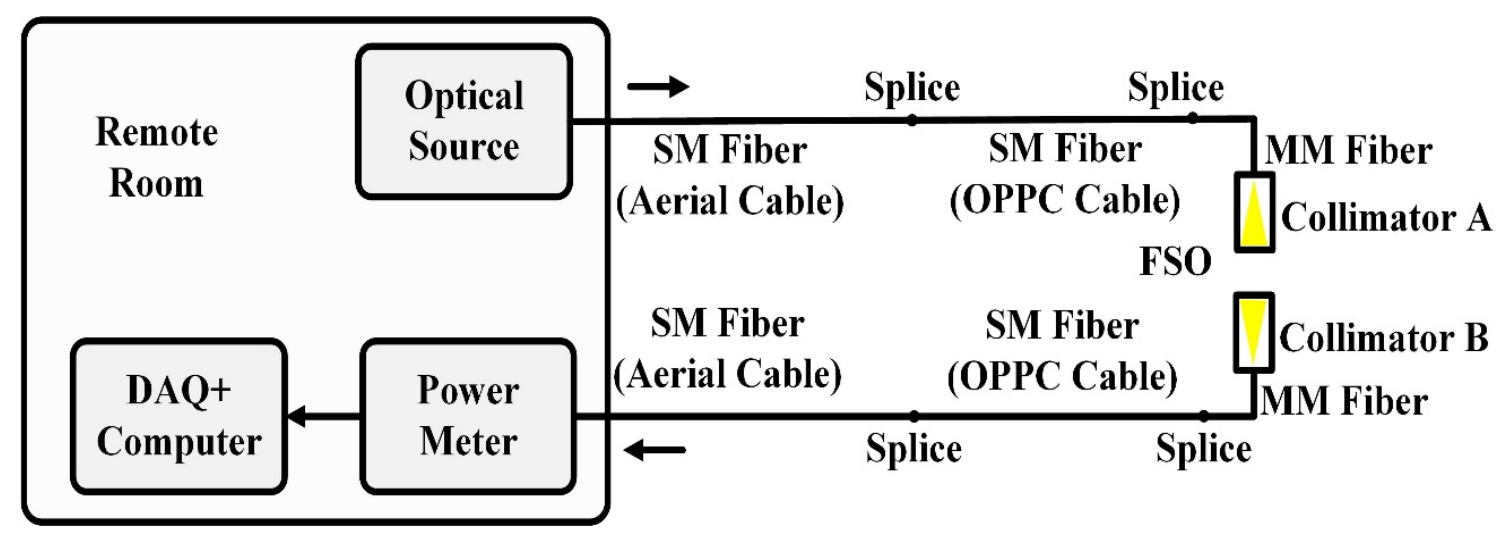

Figure 20. Block diagram of field test of the optical wireless monitoring device [77].

Considering that there is a huge potential difference between the aligners, and the typical value can be as high as $138 \mathrm{kV}$, the researchers introduced the wire post insulator and suspended it vertically from the high voltage line in the air. Meanwhile, the two collimators were fixed on the upper and lower ends of the insulator respectively to achieve insulation protection. In addition, the scheme also introduced that high power laser light power technology sent $830 \mathrm{~nm}$ light signals by multimode fiber collimator, through the wireless optical link sent to the collimator and then sent to the remote unit, and through the internal rechargeable power supply unit for the remote unit power. Refer to Figure 20 for the specific device implementation block diagram, and the monitoring device and its main components are given in Figure 21. The scheme uses wireless optical signals to transmit high-voltage sensing signals, avoiding the huge safety risks caused by wired schemes directly guiding signals from high potential to low potential, as well as the complexity of introducing customized hollow insulators to reduce risks.

In the electrical system, it is necessary to measure the current, voltage, and temperature of the high voltage overhead transmission line, which requires the special monitoring device to be completely isolated from the ground potential. Power over fiber $(\mathrm{PoF})$ includes the transmission of light energy, electronics, or electrical equipment for remote power supply. PoF has the following advantages when used in high pressure environment: no spark, lightning, electromagnetic pulse durability, can be used in extreme temperature, humidity, and other harsh environments. The PoF/OWC system and its components are shown in Figure 22. In addition, PoF can be used to replace metal cables and battery power in remote areas to improve system reliability and safety. One of the major advantages of using PoF technology in substations is that conductive copper wire does not need to be inserted in high ground areas. 


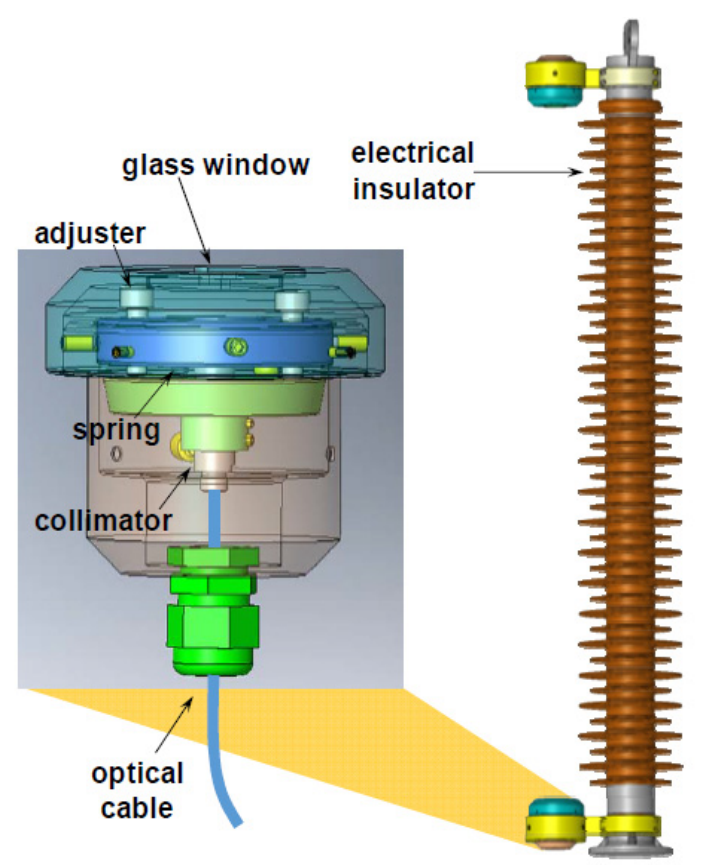

Figure 21. Optical wireless monitoring device and its main elements [77].

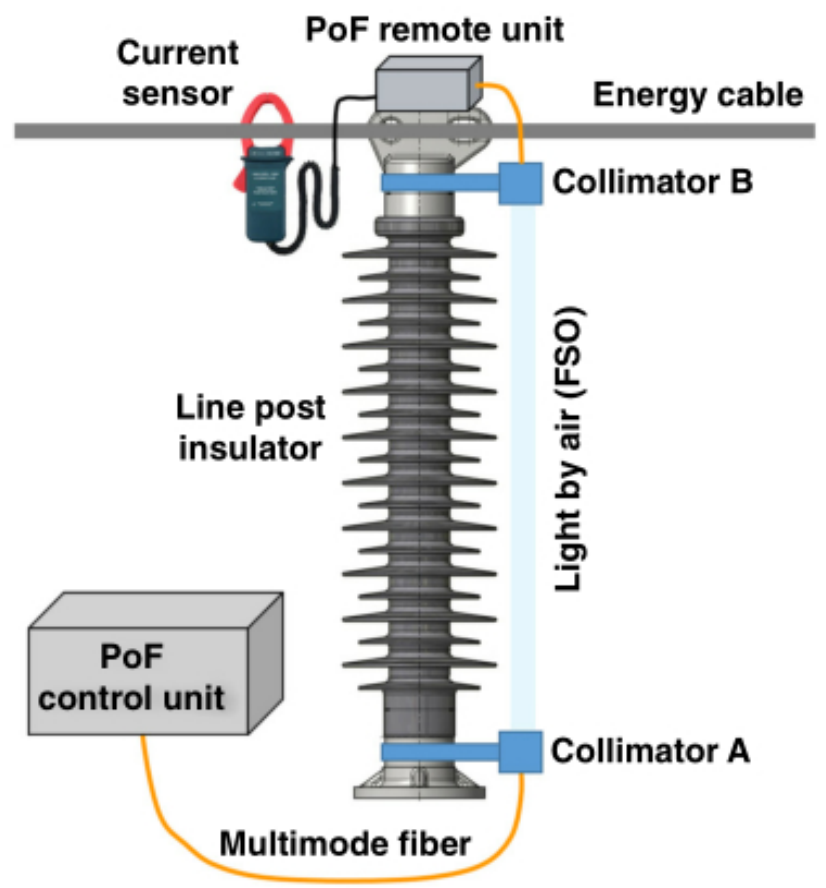

Figure 22. PoF/OWC system and its components [78].

Aiming at the above power line wireless optical monitoring system solution, the researchers conducted a prototype implementation and carried out field tests for 8 months, as shown in Figure 23. The researchers found that rainwater residing in collimator packages can significantly affect the wireless optical loss, especially the coupling loss. In addition, the experimental results show that when optical sensing technology does not use amplitude variations, but frequency or planned variations, power oscillations introduced by thermal variations will not be a critical factor.

In the laboratory testing phase, researchers also focused on the sensitivity of the above wireless optical sensing transmission system to lateral migration [77-79]. Two commercial collimator pairs, F810APC-1550 and F260APC-1550, were used in the experiment. In the experimental test of the two 
kinds of collimators, the classification test was carried out respectively under the coupling connection condition of fiber core diameter 62.5 micron multi-mode fiber and fiber core diameter 9 micron single-mode fiber. The experimental results show that compared to the small diameter collimator F260APC-1550, the large diameter collimator F810APC-1550 can support a greater degree of lateral deviation. Specifically, the collimator F810APC-1550 can support a horizontal offset higher than $3 \mathrm{~mm}$ under the constraint of not less than $-10 \mathrm{dBm}$ received optical power. Correspondingly, the collimator F260APC-1550 can only support an offset of $1 \mathrm{~mm}$. At the same time, when the large diameter collimator is combined with the multi-mode fiber, the support degree of the system to the offset can be further improved. The above results are of great practical significance for improving the wireless optical sensor transmission system in the harsh sensing environment such as outdoor high temperature and high wind for reliability and reducing the link interruption frequency and interruption duration.

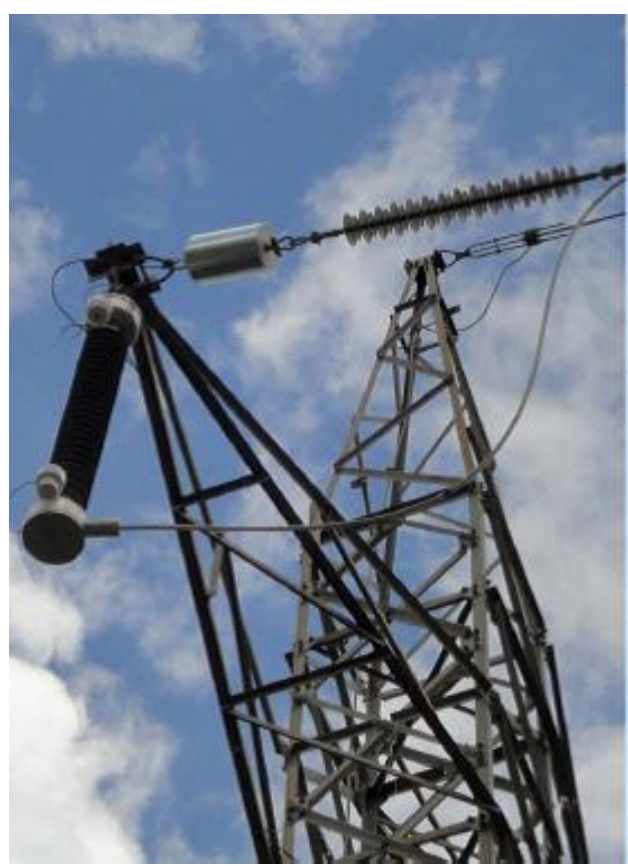

(a)

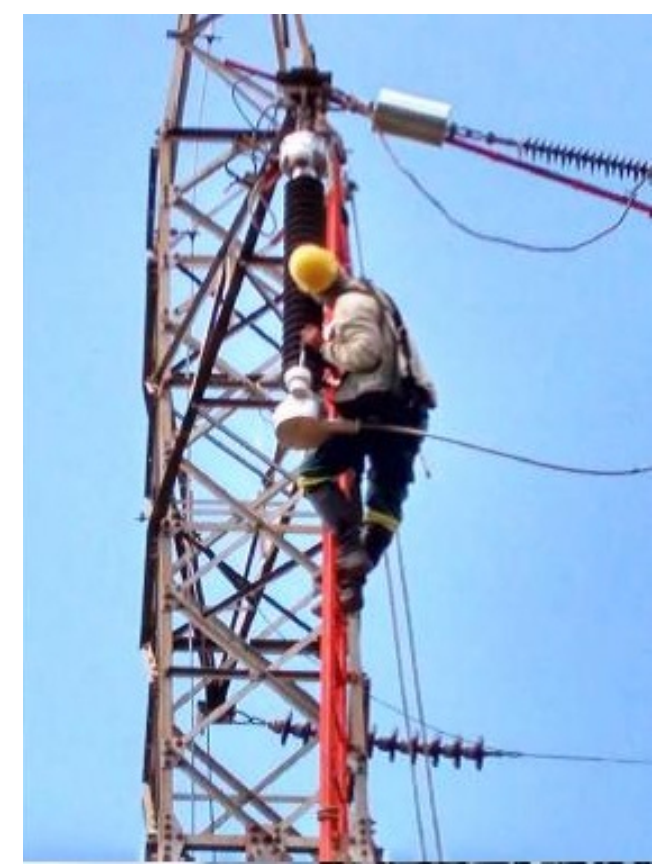

(b)

Figure 23. (a) Optical wireless (OW) device anchored in a tower of the transmission line, (b) a technician aligning the collimators on the top of the high voltage tower [77].

In [80], laboratory tests were carried out according to the scheme in Figure 24 to observe the sensitivity of the outdoor WOC system to lateral misalignment. For each type of collimator, two conditions were tested, that is, coupling with a diameter of $62.5 \mu \mathrm{m}$ through a MM fiber, and with SM fiber whose core diameter is $9 \mu \mathrm{m}$. They captured the optical power and spectral data (collimating gap $\mathrm{Z}$ passed twice), changing the transverse displacement (transverse) $\mathrm{X}$ for different distances $(\mathrm{Z})$.

In the beginning, the lateral displacement $X$ is kept under optimal conditions, and the possible coupling for each interval $\mathrm{Z}$. Then the collimator moves in the $\mathrm{X}$ direction (through symmetry, only one direction is needed). The experiment is to send a light signal through the fiber grating interrogator of the existing broa band light source, and measure: the optical power at point $\mathrm{A}$, the optical power at point $B$ before the fiber grating sensor, the optical power at point $B$, the peak value of the spectrum after the fiber grating sensor, and the values. The sensor captured the interrogator. We noticed that when only SM fiber is used, there is no need to measure the optical power of point $A$ in front of the FBG sensor, and the optical power readings of point $A$ and point $B$ coincide. 


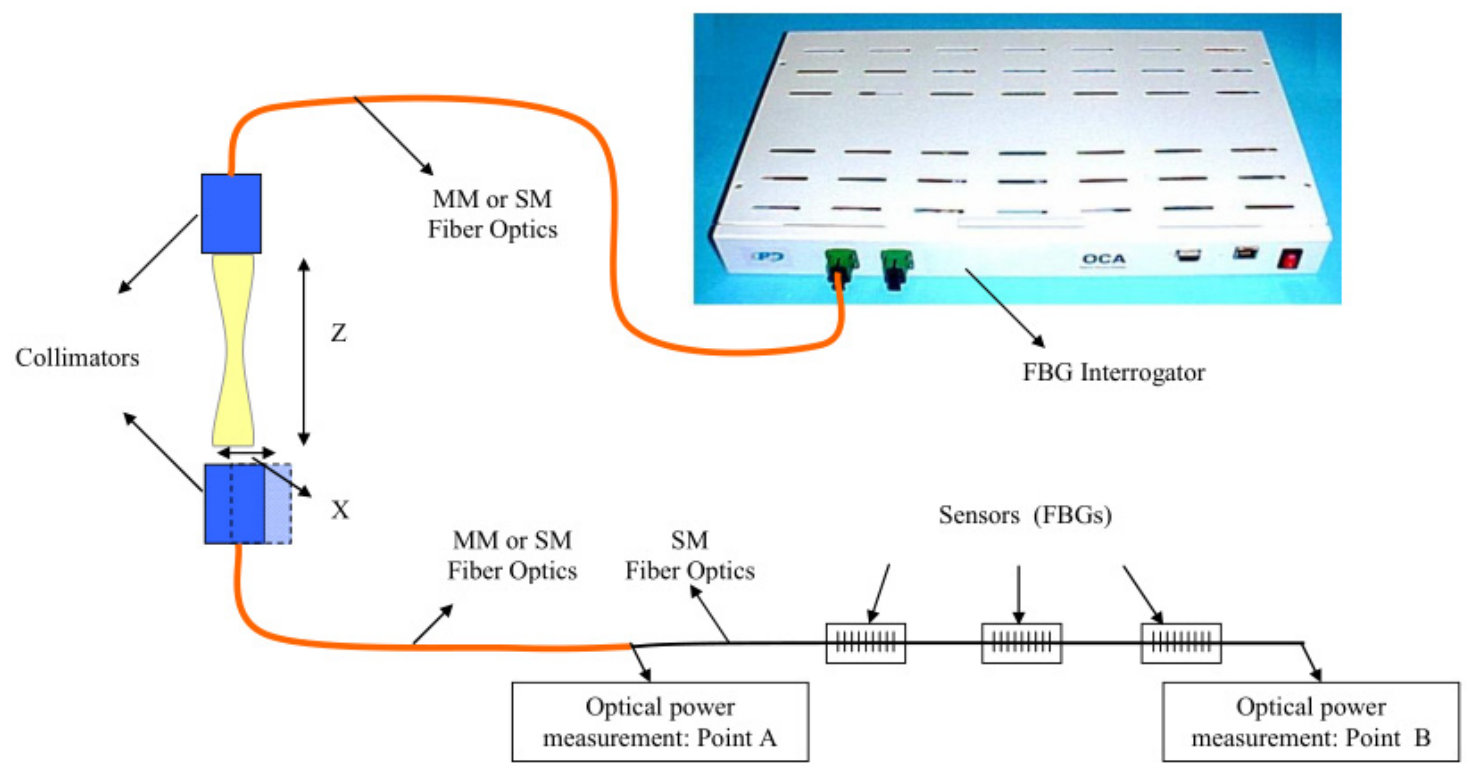

Figure 24. Experimental setup used for coupling efficiency measurement [79].

Generally speaking, it is observed that the lateral displacement supported by the largest diameter collimator (F810APC-1550) is greater than that of the smallest diameter collimator (F260APC-1550). In fact, the lateral displacement of the $-10 \mathrm{~dB}$ peak amplitude of the F260APC-1550 collimator is about 2-3 mm, while the lateral displacement of the F810APC- 1550 collimator is about $4-5 \mathrm{~mm}$. Moreover, contrary to the imagination, the use of multimode fiber does not seem to bring significant gain. In reality, for the collimator F260APC-1550, single-mode fiber supports greater lateral displacement than multi-mode fiber. Actually, with the peak value of the frequency spectrum falling at a level of $-10 \mathrm{~dB}$, about $3 \mathrm{~mm}$ requires lateral displacement and $2 \mathrm{~mm}$ of fiber $\mathrm{mm}$. However, for the collimation F810APC-1550 there is an increase in lateral displacement from 4 to $4.5 \mathrm{~mm}$ to $5 \mathrm{~mm}$ [79].

\subsection{Optical Wireless Power Inspection}

\subsubsection{Two Ways of Power Inspection}

Compared with the traditional power inspection methods, unmanned aerial vehicle (UAV) power detection line is more efficient and safer to operate in the forests, deep mountains and forests, rivers and lakes. In terms of operation precision, the UAV power line also gradually achieves the effect of accuracy that is comparable to the professional camera. UAV aerial survey could clearly study and judge the line loss parts that are hard to find by human eye. There are two main methods for power inspection: one is the visible/photoelectric pod detection carried by a multi-rotor UAV, mainly solves these problems such as insulator damage, needle, wire damage, temperature anomaly detection problems. The other is the secondary power channel data with multi-rotor/v TOL equipped LiDAR, which mainly solves the problems of tree barrier detection, cross crossing detection, tree growth analysis, power detection, and so on. Combined with the two inspection methods, how to solve the need of regular inspection and fine inspection at the same time to improve the efficiency of operation.

Compared with fixed-wing UAVs, multi-rotor UAVs fly at a low speed and have the function of hover on the fixed point. They could observe specific inspection targets in detail, which is conducive to defect discovery, such as insulator burst, wire broken strand, wire icing, bolt detection, foreign body suspension, etc. Since the defect inspection of the tower needs to observe the insulators, bolts, wires, and other special details, the traditional manual inspection has to climb up the tower to inspect with electricity, which is extremely dangerous. The multi-rotor UAV may hover around the tower and take pictures of the tower from all directions, which is undoubtedly a revolution of power inspection. 
The multi-rotor UAV inspection system consists of two parts: UAV flight platform and ground station. The flight platform includes the fuselage, power supply, propeller, flight control system, cradle head, camera, and image transmission system. The ground station includes surveillance and observation systems that monitor the UAV's flight status (such as batteries, number of satellites, flight path, altitude, etc.,) and receive high-definition video signals sent by the UAV. The operator could use this information to control the UAV to capture target images and video information in real-time; the evening after sunset and when on a cloudy day it does not rain. Generally, it is better to fly along the wire above the wire side when the plane is patrolling, and the plane and the sun should be on the same side of the wire to avoid backlight. The axis of the lens should be at an angle with the horizontal plane and less than $100 \mathrm{~m}$ away from the wire. The orientation of the lens (usually autofocus) is usually fixed on the ground in advance and does not change during flight. If change is needed, it is achieved by changing the flight path of the aircraft. If there is a very complex gyro stabilization system platform on a moving vehicle, it can realize automatic positioning and use images to accurately analyze the transmission route.

Finally, the use of autonomous satellite navigation control, geographic matching line automatic control, automatic tracking of the line tower, and flight control functions, so that the drone can patrol to follow the flight control according to the transmission line, altitude, and direction angle of the fully automatic line, making the drone close to the body patrol work on transmission lines.

\subsubsection{Optical Wireless Electric Tunnel Inspection}

In the course of tunnel inspection, power tunnel detection is a dangerous and complicated task. Since the length of the tunnel is long and relatively closed, general network signals cannot be transmitted, patrol officers may be out of touch, not to mention the real-time detection of data sent back. The inspectors hope to keep communication with the ground centralized control center. In this way, in the event of a line failure or accident, it will keep in touch with the ground centralized control center, and work together to troubleshoot and carry out on-site rescue. At the same time, since the tunnel patrol is a dangerous work, it has a strong demand for personnel positioning and navigation. However, because the cable tunnel is usually located at a depth of more than $10 \mathrm{~m}$ underground, and the influence of the structural characteristics and electromagnetic characteristics of the tunnel, all kinds of RF signals in the tunnel are seriously attenuated, which makes it difficult to satisfy the above requirements.

IWOC-PLC system could solve the above problems of substation and cable tunnel patrol. This is because when LED lights are used to communicate in substations or cable tunnels, they neither generate radio waves nor interfere with equipment in substations or cable tunnels. Using the LED as the communication node in the workstation, inspectors can always maintain a network connection with the background operating system, obtain information services and conduct confidential communication during the inspection. At the same time, it can precisely locate inspectors and the equipment they operate.

In [81], Song et al. proposed an intelligent detection network structure for substations or cable tunnels. Where the working data of the business system is transmitted through the network to the lighting LEDs in different positions in the substation or cable tunnel; the developed light source transceiver modulates the data into light signals and loads them on the LEDs for transmission. On-site inspection personnel receive VLC signals through user transceivers to realize data communication services and high-precision positioning services. At the same time, the user transceiver uses visible light signals to uplink location information and communication data to realize real-time monitoring and interaction between on-site operators and background managers.

Another possible solution is a LiFi+PLC-IoT solution. Since power tunnels lack wires the most, allowing the wires to transmit data and tunnel ceiling lights to act as transmitters form a complete solution. Wherever they go, the inspectors can send back data in real-time and talk to the people behind them in real time, at speeds currently as high as $20 \mathrm{Mbps}$. 


\section{Wireless Power Transfer of Power Industry Optical Wireless Communications}

Wired power transmission has some unsafe factors, such as line aging and tip discharge, which cannot be used on some special occasions, such as seabed and mines. In addition, cable transmission requires too many lines, which brings inconvenience to people's lives. Therefore, wireless power transfer (WPT) technology arises at the historic moment. Recent development of WPT provides a new node model for wireless sensor networks technique to extend their lifetime by the redistribution of energy [82]. It does not require a power transfer from the power supply conductors to the energy receiver technology. WPT technology development focused on applications requiring unidirectional power transmission [83].

WPT technology consists of two types: near field and far field [84]. The former is suitable for applications where the distance between transmitter and receiver is within a few millimeters or centimeters, such as handheld device charging, radio frequency identification technology, induction cooking technology, and wireless charging or continuous WPT of implanted medical devices. In [85], authors validated the use of WPT technology in medical settings and for the elderly and disabled. On the country, the latter approach allows for longer distances. It is applicable to applications where the distance between transmitter and receiver is within kilometers [86].

The development of WPT technology is mainly concentrated in application areas that require unidirectional power transmission. In recent years, bidirectional WPT (BD-WPT) systems in electric vehicles (EVs) grid integration of vehicle to grid (V2G) to realize the concept has been widely applied [83]. In order to reduce the impact caused by the intermittency of renewable energy systems and promote dynamic demand management, the V2G concept can be used as a cost-effective alternative energy storage unit. There have been many reports on the converter design, compensation topology and control technology of BD-WPT systems. One of the key issues of the Beidou-WPT system is to use external communication links to realize information sharing between main and auxiliary components. In [87], the secondary side controller is used to achieve synchronization between the two parties and control the power transmission. In [88], the author accurately modeled the BD-WPT system and proposed several methods to analyze the power flow.

In order to realize the concepts of V2G and grid to vehicle, a two-way converter between the power grid and electric vehicles is needed to facilitate the transmission of electric energy between the power grid and electric vehicles. In an electric vehicle charging system, it is necessary to ensure that the working characteristics of the converter do not affect the grid-side current. In [89], a single-phase DQ controller for grid-side bidirectional converters is proposed. In [90], an optical WPT system was proposed, which could finish fast charging over a long distance while satisfying human body safety. A single junction gallium arsenide solar cell is designed to maximize the output power of the solar cell. In the rechargeable battery of portable equipment, a step-up direct current-direct current (DC-DC) converter is used to offer a stable current and convert it to the desired DC voltage.

\subsection{Optical Wireless Power Transfer}

Recently, WPT technology has received extensive attention and has rapidly developed from a theory to commercial products [91]. The existing WPT technology can be roughly divided into transmission based on non-radiative coupling and transmission based on radiated radio frequency [92]. Transmission technologies based on non-radiative coupling include inductive coupling [93], magnetic resonance coupling [91], and capacitive coupling [94]. The technology based on non-radiative couplers has high transmission efficiency and has been diffusely applied in people's household appliances, for instance wireless chargers for smart phones. However, the effective transmission distance of non-radiative coupling technology is less than a few meters. Although RF based transmission technology could transmit power over longer distances, the transmission efficiency is inversely proportional to the square of the distance [95]. Although the transmission technology based on RF-beam forming has been proposed to increase the transmission distance [96], there is still a limit to the effective transmission distance. 
Furthermore, it can cause electromagnetic interference (EMI) to other electronic devices. To make the transmission distance longer, optical wireless power transfer (OWPT) is a feasible solution. As a wireless energy transmission technology that uses light as an energy transmitter, in OWPT, the transmitting terminal converts the electrical energy into optical power, and the receiving terminal uses an optical power receiver to receive, for instance a solar cell, to convert the received optical power into electrical energy.

Different from that of the RF beams, the size and the power of a laser beam could maintain over longer distance. Thus, the transmission efficiency of a single-wavelength laser does not decrease with the increase of distance. Moreover, it does not create EMI on existing radio communications. The paper [97] explains that the expected DC to DC transmission efficiency of WPT technology is a function of distance. Although OWPT is less efficient over short distances than other technologies, it is expected to be more efficient over long distances. So far, there have been many studies on OWPT [90,98-102]. Most of them are based on a laser emitter and a solar cell receiver, while some OWPT uses a LED transmitter that has been proven $[98,99]$. Although OWPT is a solution suitable for long-distance wireless power transmission, research on OWPT is still rare.

In the experiment of [103], similar to other traditional OWPT work, authors make a LD as an optical power transmitter. However, they proved that both a PD and a solar cell could be used to receive light power. After that, the PD and solar cells as the performance of the optical power receiver also were compared. Figure 25 illustrates the construction of OWPT. At the transmitter, as the energy is usually electrical energy, a light source (such as laser, LED, etc.) is needed to convert the electrical energy into optical power. When transferred to the light source of electricity for $\mathrm{P}_{\mathrm{Et}}$, and light source the light output power for $\mathrm{P}_{\mathrm{Ot} 1}$, the electric-to-optic $(\mathrm{E} / \mathrm{O})$ power conversion ratio of the optical source is $\mathrm{C}_{\mathrm{E} / \mathrm{O}}$.

Transmitter site

Receiver site

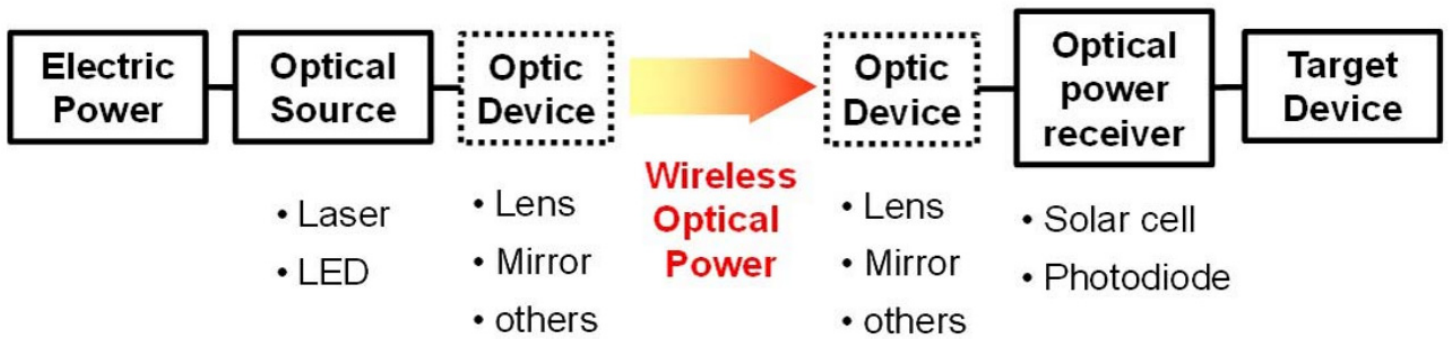

Figure 25. System block diagram of OWPT [100].

In [104], Kim et al. demonstrated underwater OWPT using LD as power transmitter through experiments. They studied the properties of solar cells and PD as power receivers. They also optimized LD, PD, and solar cells for maximum transmission efficiency. The maximum transmission efficiency of back-to-back OWPT measured with the PD receiver was 4.3\%. They then demonstrated OWPT in tap water and seawater. The result shows that the attenuation in sea water is $3 \mathrm{~dB} / \mathrm{m}$.

The application of OWPT in indoor environment was described in [104,105]. In these studies, ambient light was determined to be negligible, so the concept of OWPT to SCs was investigated under dark conditions. In [104], by using high brightness white LED, a collimating large parabolic mirror and a-Si solar panel placed at $5 \mathrm{~m}$, an OW link was created. Measure the maximum link efficiency and harvest power were only $0.1 \%$ and $18.3 \mathrm{~mW}$, respectively [100].

Raavi and his researchers in [90] proposed a solar cell wireless light energy transmission system based on optical antenna, as detected in Figure 26. As can be shown, the light source is located on the ceiling of the room and emits light signals that comply with the US Federal Communications Commission (FCC) human safety regulations which is less than $1 \mathrm{~mW} / \mathrm{cm}^{2}$ [89]. The central station connected with the light source receives the guide signal from the equipment to be charged and controls the activation and deactivation of the light source. The mobile device to be charged contains an optical 
antenna array and a DC conversion circuit integrated on top of the solar cell, which can be anywhere in the house to receive energy from the light source. The system will realize a safe and efficient intelligent charging system.

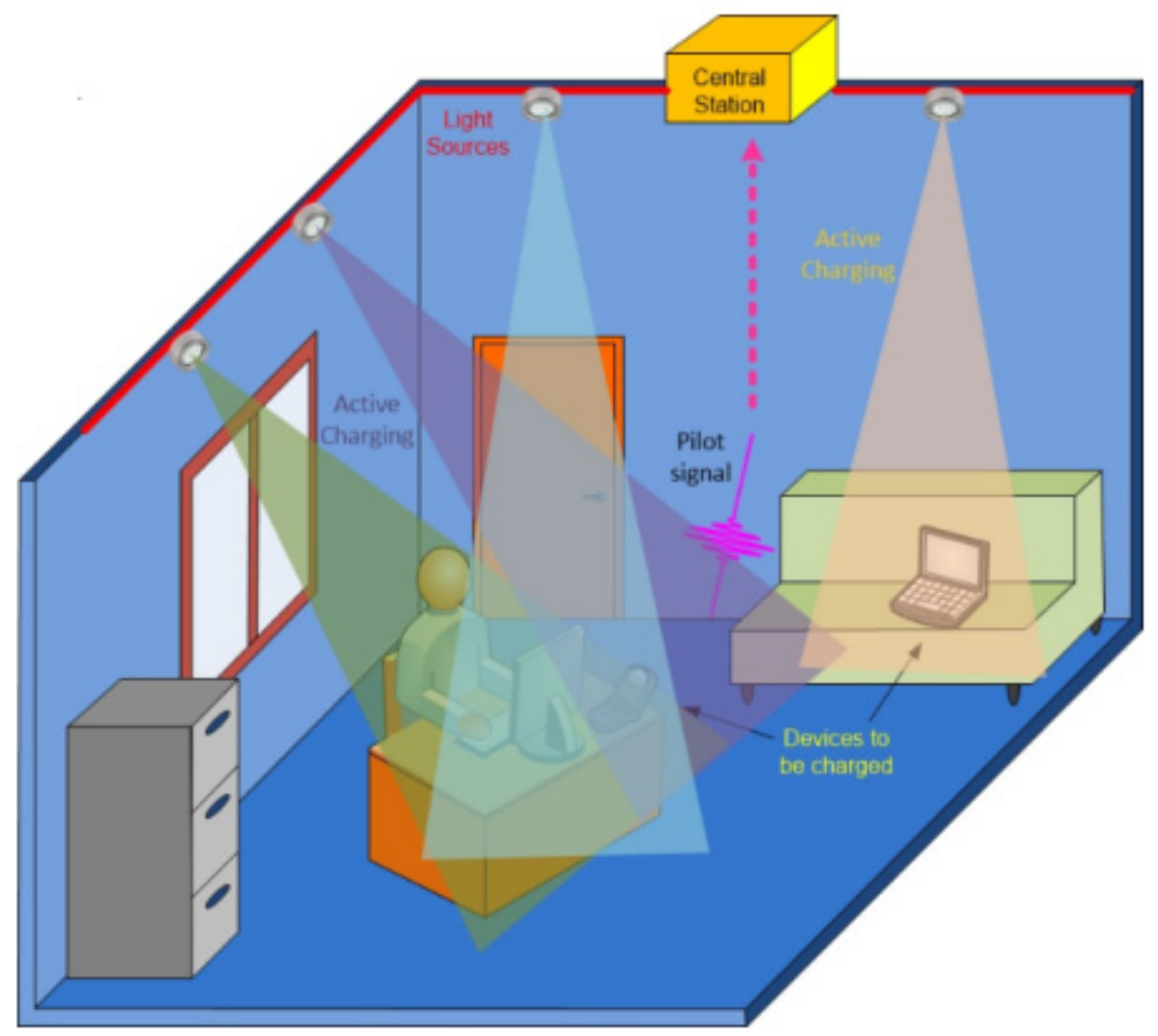

Figure 26. Diagram of the proposed wireless energy transfer system [90].

In order to realize this system, a nano-structured optical antenna using the enhancement effect of plasmonic electric field was designed and analyzed [90]. In addition, an efficient single-junction GaAs solar cell was designed to maximize the output power of the solar cell. In the rechargeable batteries of portable devices, a step-up DC-DC converter is used to provide a stable current and convert it to the required DC voltage. Because the optical antenna can enhance the plasma electric field effect, thereby promoting the efficiency of the energy transfer system without conflict with human safety rules. They simulated the realization of optical antennas, solar cells, and DC-DC conversion circuits. On the basis of this simulation, the solar cell output of the proposed technology is 40 times higher than that of the traditional solar cell system.

\subsection{Optical Wireless Power Transmission with Mirror}

The OWPT system consists of a power transmitting unit (light source), a power receiving unit (solar battery), and a DC operating circuit. Generally, a light source such as a laser converts electrical energy into a highly collimated beam, which can travel within a few meters with very little loss. Besides, solar cells convert light energy into electrical energy. The light source is driven by a DC working circuit to output a specific and stable solar battery voltage [106]. On this basis, a compact system can be used to achieve long-distance transmission, and no electromagnetic waves will leak into the environment outside the beam. By using this method, when the irradiated area is larger than the solar cell, light will be wasted and the power transmission efficiency will be reduced. In addition, when the solar cell area is larger than the illuminated area, the conversion efficiency will be reduced 
because the solar cell is not fully irradiated at this time. Therefore, to maintain high efficiency, an appropriate irradiation pattern is required [107].

To improve the efficiency of the transfer system OWPT in remote and steep, authors propose a new system image OWPT numerical and experimental investigation and analysis that put forward the divergence angle of the power generation efficiency and system variables in the model space of the beam [106]. Furthermore, the OWPT system with multiple mirrors was considered, and the number of mirrors required for rooms of different sizes was studied. In this paper, the beam can be transmitted through the mirror to a device far away from the light source and with a small inclination angle, which avoids the energy waste caused by the beam shining on the outside of the solar cell and improves the energy transmission efficiency [106]. Moreover, the experimental results show that the WPT system using mirrors can effectively extend the application range of OWPT system and improve the flexibility of room effective charging [106].

\subsection{Other Technologies of Wireless Power Transfer}

\subsubsection{Optical Power Transmission}

Optical power transmission (OPT) is one of the least studied technologies for wireless transmission of power to biomedical implants [107-111]. In the literature, there are few examples of OPT, even in other applications $[88,90,101,112]$. An external $T_{X}$ unit in a complete OPT system uses an external laser to power the implant. The light emitted by the external laser is received by the CMOS PD array implanted under the skin and converted into electrical energy, using the power management unit. The advantage of this WPT method is to provide a compact solution, because the CMOS-based power receiving system can be smaller than other systems [100]. The authors suggest in [111] that implantable sensors could be used for short-term in vivo monitoring after surgery, and that the use of biodegradable materials could avoid secondary surgery to remove the devices. Therefore, OWPT is considered to be applied in human skin to complete communication with a broad prospect.

Compared with other methods, the OPT system for biomedical implant devices is still in the early stages of development, and the potential of this technology depends on the results of further research. In addition to the design and manufacturing challenges, more system-based implementation challenges need to be solved before considering using the system for complex implants. OPT requires a complete analysis of organizational security. Furthermore, misalignment and limited output power are important issues established by OPT in biomedical implant applications.

OPT is the least researched WPT technique presently. To support most IMDs, PTE is much lower. Besides, any misalignment of Rx photodiodes with $\mathrm{T}_{X}$ can significantly reduce PTE. Moreover, WPT technology still requires a rigorous organizational security analysis before its use in IMDs can be considered.

The generation ratio of OWPT system with reflector is obtained when the reflector is 95\% [106]. Figure $27 \mathrm{~b}$ shows the power generation ratio of the system under a light source of 5 square $\mathrm{mm}$. It can be seen that within the range of $150-650 \mathrm{~cm}$, the power generation ratio can reach more than 0.7. Combined with Figure 27 a, the result shows that the energy transfer efficiency above 0.7 can be achieved within a distance of $650 \mathrm{~cm}$ after the installation of the reflector, which causes the extension of the effective distance. Furthermore, when the distance is between $150 \mathrm{~cm}$ and $300 \mathrm{~cm}$, the beam is transmitted more flexibly through direct irradiation of light source and indirect irradiation of mirror, even if the direct beam is blocked by obstacles. In this system, the reflection loss is a disadvantage because of the need of multi-plane mirror module. 


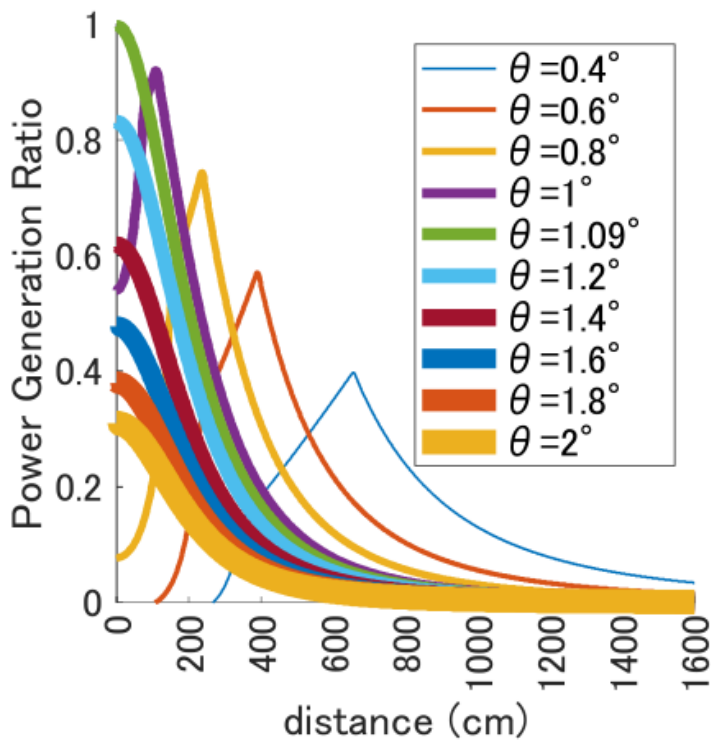

(a)

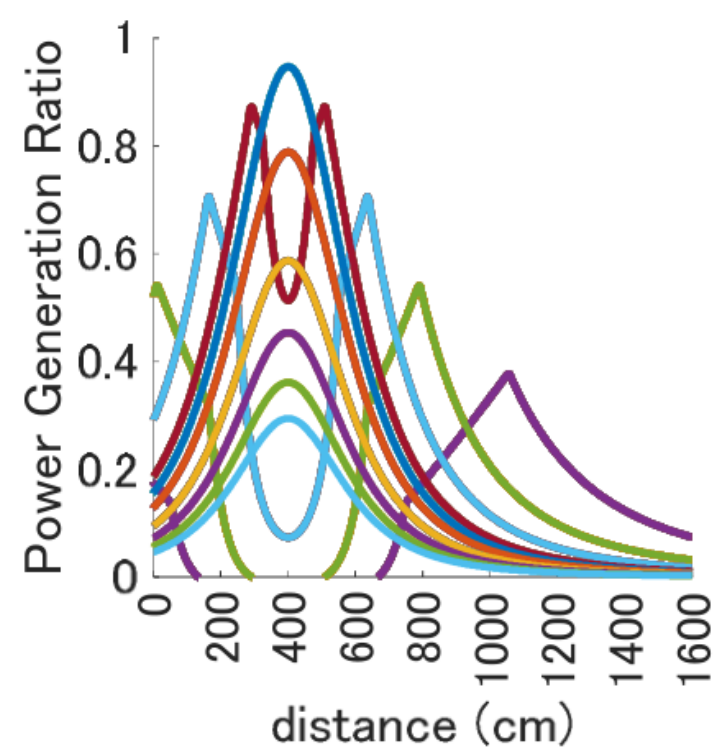

(b)

Figure 27. Power generation ratio obtained: (a) irradiated directly by light source; (b) irradiated by mirror [106].

\subsubsection{Laser Emission Wireless Power Transfer}

Laser WPT technology research is moving toward the high transmission power, high transmission efficiency, and long transmission distance development [113]. The transmitting part of laser transmitting WPT system mainly includes laser driver, laser and optical transmitting antenna. The laser is a key part of laser emission. Lasers with a wavelength of around $800 \mathrm{~nm}$ are known to be better suited for wireless energy transmission. Currently available high-power lasers with a wavelength of approximately $800 \mathrm{~nm}$ include solid-state lasers and semiconductor lasers. As for conversion efficiency, solid-state lasers only reach $40 \%$, while semiconductor lasers can reach $55-60 \%$. Semiconductor lasers have the advantages of high electro-optical conversion efficiency, small size, light weight, and low cost. In recent years, the electro-optical conversion efficiency of AlGaAs semiconductor lasers has been improved steadily. Experiments show that the efficiency can reach $70 \%$ and will be higher in the future [114]. The transmitting antenna of laser-transmitting WPT can be designed to approach the diffraction limit and obtain the minimum receiving point as far as possible. In order to reduce the influence of receiving aperture and increase the distance, it is necessary to reduce the emission angle of laser beam.

Thus, in the transmitting system, it is usually necessary to converge the launching lasers and achieve the requirements of the collimation system through beam forming launching technology. Kim et al. studied the wireless optical energy transmission based on optical beam forming, and the beam forming technology realized by the spatial light modulator realized wireless energy transmission [98]. In order to support the power and distance requirements of the Internet of Things and mobile devices, literature [115] proposed a distributed laser charging (DLC) system. The self-aligning feature of DLC provides a more convenient way to charge the Internet of Things and mobile devices without specific positioning or tracking, as long as the transmitter and receiver are within LOS of each other. In 2017, Japan in [116] put forward a scheme in which a photovoltaic cell is fixed on the top of the car and a solar-pumped laser is used on both sides of the road to receive the solar lamp and directly convert it into a laser, and then the laser is irradiated to the photovoltaic cell to provide energy for the car, as shown in Figure 28 [113]. 


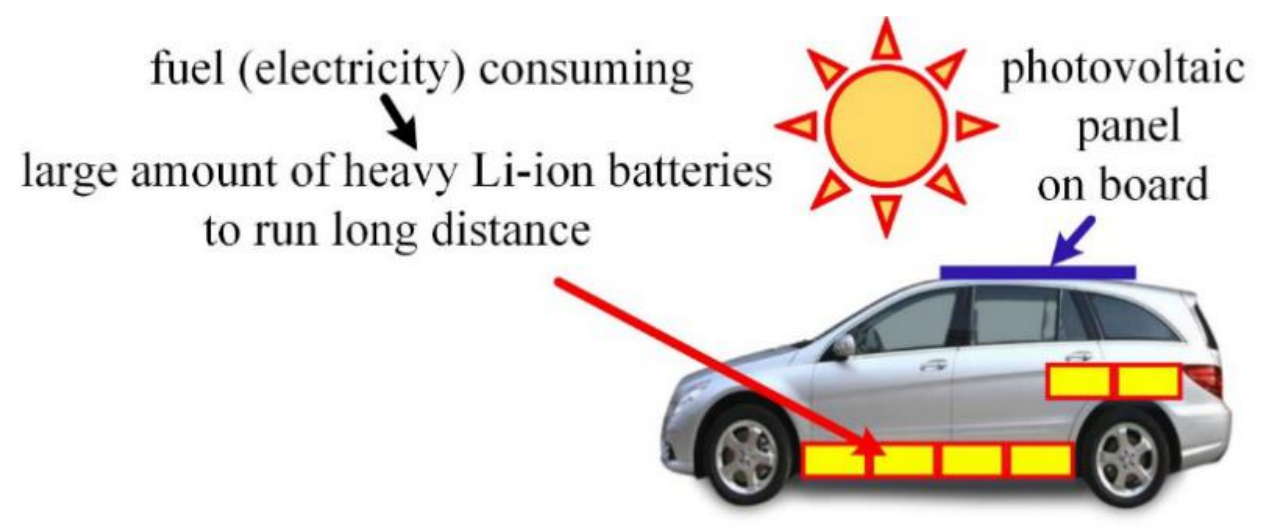

(a)

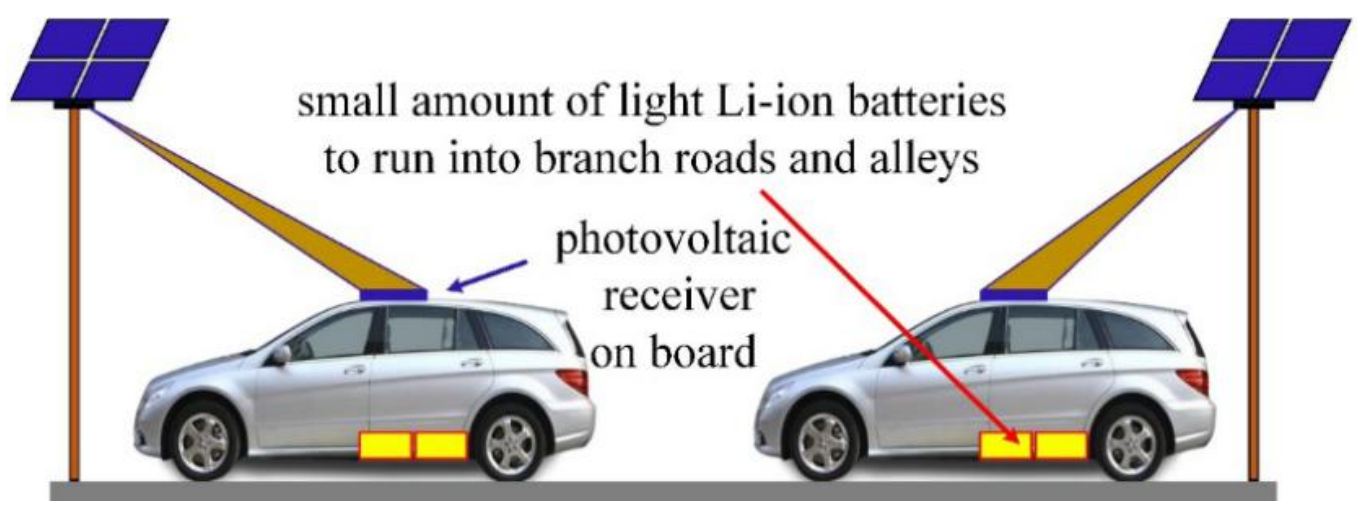

(b)

Figure 28. The solar panels on the roof of electric vehicles (EVs) receive laser light from infrastructure along arterial roads; (a) sunlight illuminates directly at photovoltaic cells on car roof; (b) photovoltaic cells on the car roof illuminated by laser generated from solar-pumped [113].

\subsection{Dynamic Optical Wireless Power Transfer}

OWPT is one of the most appropriate ways to extend the transmission distance while keeping the relevant system components relatively simple [117]. Some companies, for example, [118], are already investigating the technology for use in consumer electronics and other areas. At the same time, some research institutions and institutions around the world are conducting far-field WPT research on OWPT, such as $[119,120]$.

An OWPT system consists of three parts: the transmitter of light, transmitting environment, and receiver of light. Therefore, the OWPT system efficiency is deduced from [98], that is:

$$
\lambda_{\mathrm{OWPT}}=\lambda_{T} \times \lambda_{\mathrm{C}} \times \lambda_{R}
$$

where $\lambda_{T}$ stands for the light transmitter energy efficiency, which is for the ratio of output energy to consumed energy. $\lambda_{C}$ is the optical transfer through the environment, and shows how much energy is lost when light is traveling in the considering environment such as air, pure water or sea water, etc. $\lambda_{R}$ of the optical receiver; respectively. The correctness of the formula is verified by experimental tests and could be used to explain the measurement efficiency of OWPT system.

To verify the proposed dynamic optical WPT concept, Nguyen et al. first created a simple experiment, as shown in Figure 29. Here, solar energy is collected and stored in a device including DC-DC and DC-alternating current converters, and Li-ion batteries. Then utilizing the device to power the LED light bulbs of a small EV. This LED bulb consumes $8 \mathrm{~W}$ electricity as a light emitter, while a small solar panel is connected to another toy electric car as an energy receiver. The LED-solar panel 
WPT system introduced could work as far as $5 \mathrm{~cm}$. The maximum WPT efficiency is $2 \%$ when the distance is almost zero, and the maximum WPT efficiency is $1.57 \%$ when the distance is $3 \mathrm{~cm}$ [117].

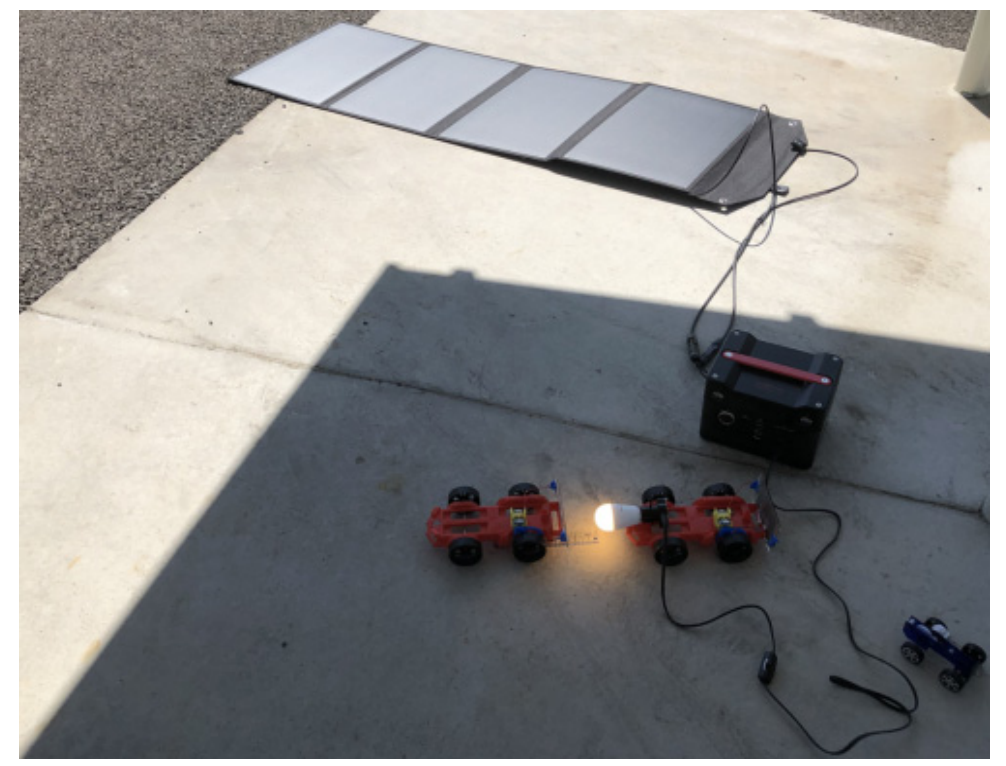

Figure 29. Experiment setup for dynamic optical wireless power transfer (WPT) between ground EVs using visible light [117].

Figure 30a shows the situation where the blade is at a standstill without WPT, while Figure 30b shows that after the LED bulb moves to a certain distance, WPT does occur due to the rotation of the blade. Similar to the testing of ground vehicles, the dynamic OWPT system of this UAV is inefficient. Since its instrument is simple, it is predictable.

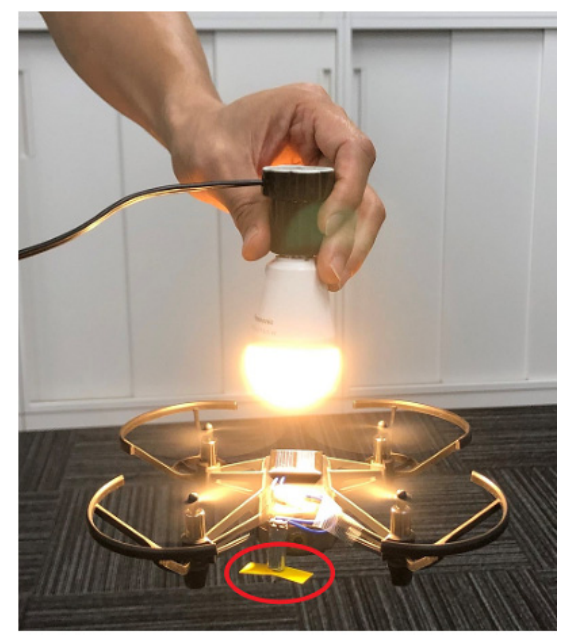

(a)

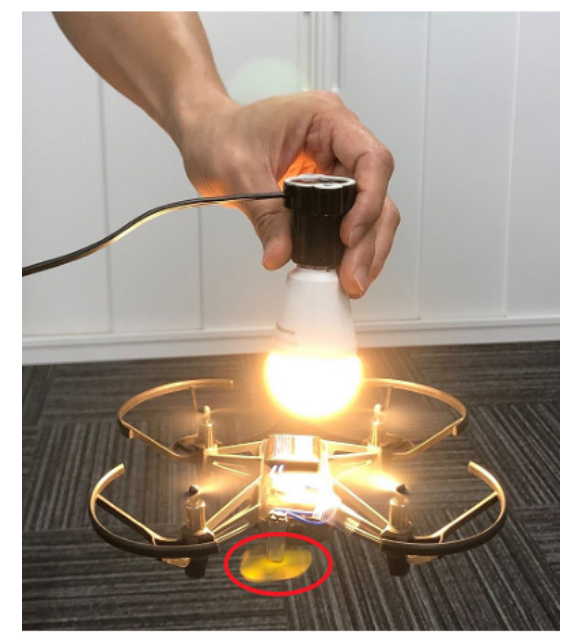

(b)

Figure 30. Experiment setup for demonstration of dynamic OWPT between a moving light source and a moving object: (a) no WPT; (b) with WPT [117].

Described above experimental OWPT efficiency is very low, it can also be seen in other systems, for instance used in underwater systems [121]. However, using high quality solar cells and light sources, higher OWPT efficiency can be expected. Theoretically, the upper limit of the energy conversion efficiency of traditional single-junction solar cells is $30 \%$. This is because most of the solar energy that impacts the battery is not absorbed or converted into heat. Scientists are studying various solar cell 
designs to eliminate these limitations in conversion efficiency and reduce energy loss. The current world record for four-junction solar cells is 46\% [122]. In [123,124], the GaSb-based solar cell designed by researchers at George Washington University in 2017 has a photoelectric conversion efficiency of $44.5 \%$. If the energy conversion efficiency of solar cells exceeds $50 \%$, it will have a great impact on the cost of power generation [122]. This is still far behind the efficiency of resonant inductively coupled systems, but is acceptable in certain applications, for example, wireless charging for consumer electronics devices.

In recent years, optical wireless technology has become more and more important. Check the freepatantsononline website about OWC, IWOC, PLC patents, there are as many as several million in each field, enough to see that optical wireless technology is widely used in today's society, which is a research hotspot. There are also hundreds of thousands of patents related to power industry and optical wireless communication, indicating that the combination of power industry and optical wireless technology is a feasible solution.

Similarly, according to CNKI statistics, there are 200 Chinese patents related to optical wireless communications from 1986 to 2020. Furthermore, Chinese patents related to visible light communication amounted to nearly 14,000, with an average of more than 1000 patents per year in the past eight years. It covers the power industry, telecommunications technology, computer software and computer applications, automation technology, radio electronics, and other multidisciplinary fields. There are also nearly 10,000 patents related to PLC, with an average of more than 800 articles per year in the past eight years. In addition, Chinese patents for the combination of visible light communications and PLC can be traced back to 2011. There are still relatively few patents on the combination of smart power and wireless optical technologies. Optical wireless and wireless optical have always been used by researches in the communication field, meanwhile power grid and smart grid belong to the categories of the power industry. Therefore, on the World Intellectual Property Organization (WIPO) official website we searched "smart grid," "optical wireless," "power line," "visible light communication," "free space optical," "power grid," and so on, and realized the search of related patents in the field of power optical wireless from 2012 to 2020, and obtained 45 patents with these themes, as shown in Figure 31 [125]. It can be seen from the figure that in the past five years, power industry optical wireless technologies have been more and more widely used. Therefore, exploring the power technology under optical wireless technologies has great development prospects.

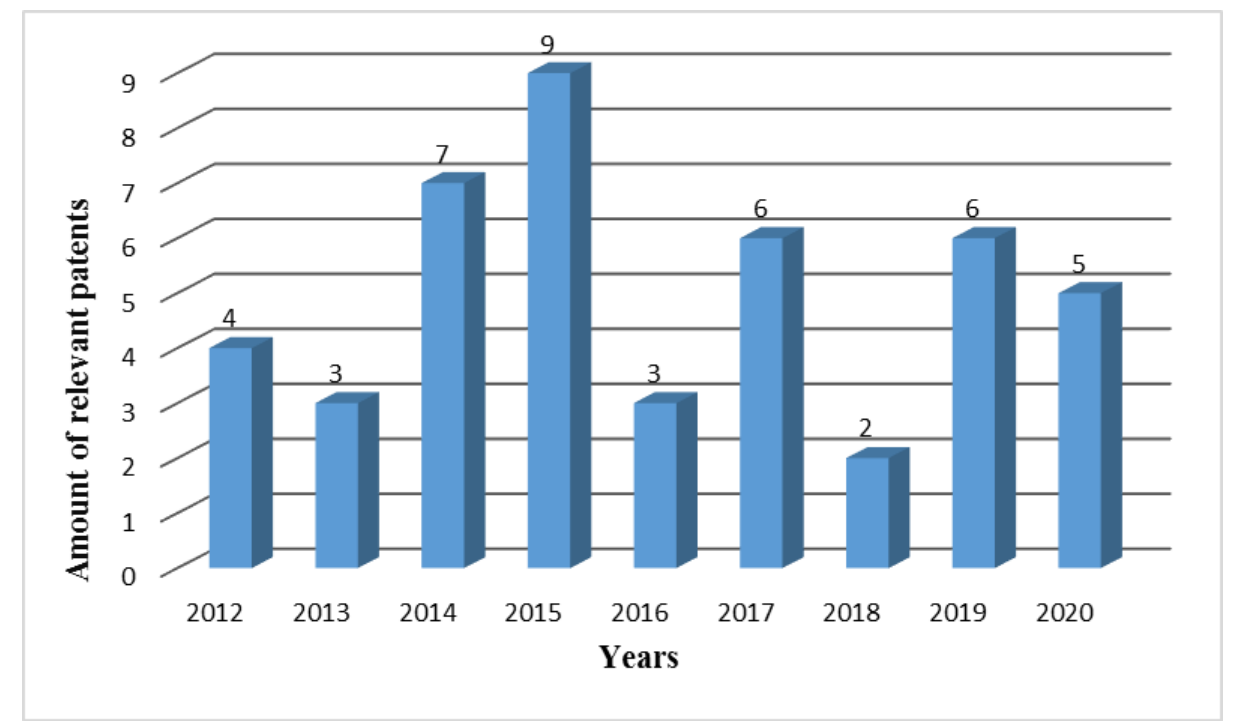

Figure 31. 2012-2020 statistical analysis chart based on power industry optical wireless patents from WIPO [125]. 


\section{Challenges and Potential of Power Industry Optical Wireless Communications}

At present, optical wireless technology for smart power is still in the broadcast technology paradigm. In this case, the power line transmits data from the data source to the data driving module, and the data driving module loads the same data stream on all light sources, and broadcasts the signal to the area covered by the light signal through the light source. This means that even if there are multiple distributed light sources, the data transmission potential of each light source cannot be fully released. In view of these problems, it is feasible to introduce MIMO technology into optical wireless technology. The basic idea is to realize multiple data streams separately to multiple distributed sources through the indoor power line network. The light source completes the loading of each data stream through its own efforts and transfers the optical signal from different data information. At the receiving end, the optical signals from different light sources are converted into electrical signals by multiple detectors, and then the original multiple streams are restored through various demultiplexing techniques.

Theoretically, MIMO technology paradigm can double the transmission capacity of the system. It must be pointed out that, unlike the radio frequency MIMO technology in the current commercial cellular network, the wireless optical MIMO technology based on the indoor distribution network must comprehensively consider the MIMO characteristics of the distribution network itself [126-129]. Generally speaking, mainstream alternating current power supply networks use a three-phase power supply. How to eliminate the transmission interference between the neutral line, the live line, and the ground line may become the key to the practical application of high-power optical wireless technology in MIMO technology. In addition, since OMA only allocates a single wireless resource to one user, such as by frequency or time, NOMA scheme allows multiple users to use the same resource. In some of the scenes, such as the near-far effect scenario and the scenario of multi-node access with wide coverage, NOMA approach using power multiplexing has obvious performance advantages over traditional OMA and is more suitable for future system deployment.

In the field of power industry optical wireless channel modeling and characterization, the existing research works cannot fully cover the diverse power grid configurations at home and abroad, nor can it be applied to the extensive non-Lambert wireless optical link configurations. Existing PLC studies show that due to the different standards of power industry, the transmission characteristics of smart grid in different countries and regions are obviously different, and it is difficult to use a single model to represent [128-130]. At the same time, as the original function of the electric power network is not used for data transmission, power grid load and electricity demand in the different periods have an obvious dynamic difference; these factors make the electric model to be further points area, the scene, the difference of the period of time to discuss, and integrate wireless optical link corresponding channel measurement and statistical analysis. Meanwhile, to meet the diverse lighting needs of roads, assembly halls and exhibition halls, the actual commercial LED light sources usually need to use the encapsulation lens technology to distribute the light beam, improve the light efficiency and improve the lighting uniformity [131-133]. This kind of technology processing results in the beam of LED light that no longer match the Lambert model in the existing theoretical analysis, and ultimately leads to the reduction of the accuracy of the power wireless optical modeling analysis results, and even the analysis of the practical significance of modeling representation. Therefore, in the next power wireless channel characteristic study, light must consider electric model, and the integrated lambert the diversity of the respective characteristics of the beam, carried out a series of specific power optical wireless channel measurements, and then through data fitting, statistical analysis methods, for instance, constructing can face a series of typical scenario high reliable power wireless optical channel model, which provide a basis for comparison in a variety of technical scheme evaluation.

In the reliability of power optical wireless link, the existing technical scheme and prototype system still have great room for improvement. Especially in the part of optical wireless link, as the light source often needs to meet the requirements of lighting function, the semi-power of the beam is relatively large, which makes the optical signal emitted by the light source unable to focus on the local spatial position where the data receiver is located [134-136]. At the same time, a considerable part of the 
signal power is projected to the space where there are no users, resulting in the actual waste of data capacity and reducing the overall energy efficiency of the system. In future research, beam forming technology can be tried to be introduced into the field of power wireless optical technology [72,137]. With the user location information fed back by the uplink, the remote data source loads the user data stream onto multiple light sources adjacent to the user space location by selecting the power line path. At the same time, the algorithm is designed to scale the above light source to different degrees, and on the premise of maintaining the same energy consumption level, light signal energy is concentrated in the area where the user is, so as to effectively enhance the user's business experience and improve the overall energy efficiency of the system.

Another outstanding disadvantages of OWPT is that it cannot be transmitted over a long distance. Because of its mobility and flexibility, UAV is used in the new technologies such as monitoring and emergency rescue and military applications. The endurance of UAV is always an urgent problem to be solved. The possibility of using OWPT technology to power UAV systems has recently been recognized as a very attractive approach that could lead to alternative and effective charging solutions [84,137-141]. Since the OWPT technology does not require electricity, and then it makes future communications technology possible to a large extent. In addition, there are few researches on OWPT, so it is a feasible scheme and has a broad prospect to consider the combination of OWPT technology and power industry.

\section{Conclusions}

With the further deepening of the demand for information and communication technology in the electric power industry, the emerging wireless optical technology will show its natural advantages in the traditional electric power industry in terms of large transmission capacity, strong confidentiality, and free from electromagnetic interference. Most of the existing researches focus on the theoretical level, so it is necessary to accelerate the practice and large-scale application of wireless optical technology in the power industry. Further, future work should focus more on the integration of power lines and indoor wireless optical transmission of high-speed, highly reliable two-way communication. With the key enabling technologies such as multi-input and multi-output, Non-Lambert beam characterization, beam forming, and wireless power transfer technologies, the transmission and coverage performance of power industry optical wireless technologies will be significantly improved and play a significant role in the construction of smart power and power industry.

Author Contributions: Conceptualization, J.D. and W.L.; methodology, J.D. and W.L.; validation, J.D. W.L., and C.-L.I.; formal analysis, J.D. and W.L.; investigation, J.D., W.L. C.-L.I., H.Z., and H.M.; writing-original draft preparation, J.D. and W.L.; writing-review and editing, J.D., W.L., and H.M.; supervision, J.D., W.L., C.-L.I., and H.Z. All authors have read and agreed to the published version of the manuscript.

Funding: This work was supported in part by the National Natural Science Foundation of China (Grants No. 62061043), Natural Science Foundation of the Xinjiang Uygur Autonomous Region (Grants No. 2019D01C020), High-level Talents Introduction Project in Xinjiang Uygur Autonomous Region (Grants No. 042419004), Tianchi Doctor Program of the Xinjiang Uygur Autonomous Region (Grants No. 04231200728), Natural Science Foundation of Xinjiang University (Grants No. 62031224624), National Natural Science Foundation of China (Grants No. 61401420), and Postgraduate Research and Innovation Project of Xinjiang Uygur Autonomous Region (Grants No. XJ2020G063).

Conflicts of Interest: The authors declare no conflict of interest.

\section{Abbreviations}

The following abbreviations are used in this manuscript:

$\begin{array}{ll}\text { AF } & \text { Amplify and Forward } \\ \text { APD } & \text { Avalanche Photodiode } \\ \text { BDM } & \text { Bit Division Multiplexing } \\ \text { BD-WPT } & \text { Bidirectional Wireless Power Transfer } \\ \text { BER } & \text { Bit Error Rate }\end{array}$




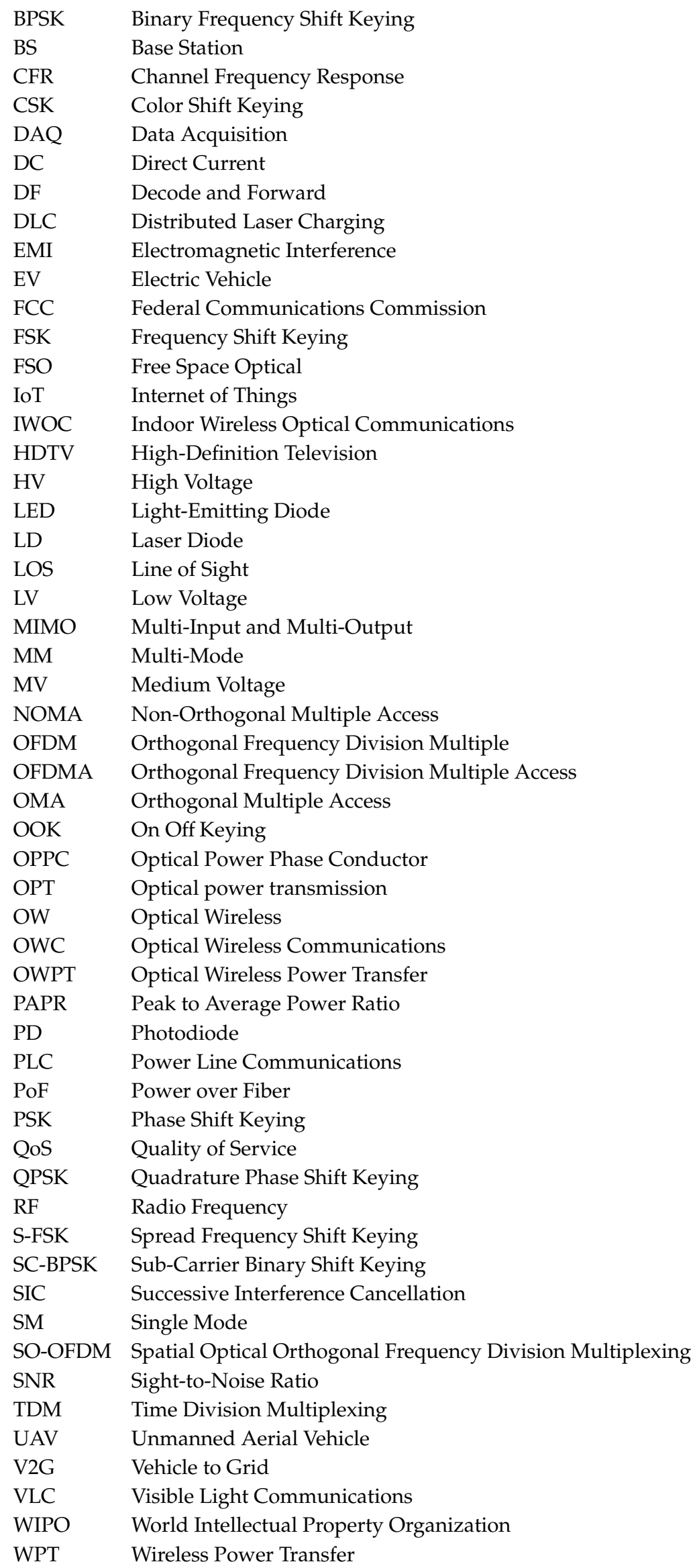




\section{References}

1. Komine, T.; Nakagawa, M. Integrated system of white LED visible-light communication and power-line communication. IEEE Trans. Consum. Electron. 2003, 49, 71-79. [CrossRef]

2. Song, X.; Wang, M.; Xing, S.; Zhao, Z. Progress of orthogonal frequency division multiplexing based on visible light communication. Laser Optoelectron. Prog. 2018, 55, 100-109.

3. Jia, K.; Zhang, S. Influence of multiuser interference on performances of direct-current-biased optical orthogonal frequency division multiplexing code division multiple access system for visible light communication. Laser Optoelectron. Prog. 2019, 56, 110604.

4. Yang, Y.; Jiang, M.; Zhang, Y.; Zhang, H. Design of full duplex visible light communication system based on single light source. Laser Optoelectron. Prog. 2019, 56, 010603. [CrossRef]

5. Ding, J.; Chih-Lin, I.; Zhang, C.; Yu, B.; Lai, H. Evaluation of outdoor visible light communications links using actual LED street luminaries. In Chinese Conference on Biometric Recognition; Springer: Cham, Switzerland, 2018.

6. Luo, J.; Fan, L.; Li, H. Indoor positioning systems based on visible light communication: State of the art. Commun. Surv. Tutor. IEEE 2017, 19, 2871-2893. [CrossRef]

7. Chowdhury, M.Z.; Hossan, M.T.; Islam, A.; Jang, Y.M. A comparative survey of optical wireless technologies: Architectures and applications. IEEE Access 2017, 6, 9819-9840. [CrossRef]

8. Tsonev, D.; Videv, S.; Haas, H. Towards a $100 \mathrm{~Gb} / \mathrm{s}$ visible light wireless access network. Opt. Exp. 2015, 23, 1627-1637. [CrossRef]

9. Khalighi, M.A.; Uysal, M. Survey on free space optical communication: A communication theory perspective. IEEE Commun. Surveys Tuts. 2014, 16, 2231-2258. [CrossRef]

10. Ndjiongue, A.R.; Ferreira, H.C.; Song, J.; Yang, F.; Cheng, L. Hybrid PLC-VLC channel model and spectral estimation using a nonparametric approach. Trans. Emerg. Telecommun. Technol. 2017, 28, 1-13. [CrossRef]

11. Nlom, S.M.; Ndjiongue, A.R.; Ouahada, K. Cascaded PLC-VLC channel: An indoor measurements campaign. IEEE Access 2017, 6, 25230-25239. [CrossRef]

12. Kashef, M.; Abdallah, M.; Al-Dhahir, N. Transmit power optimization for a hybrid PLC/VLC/RF communication system. IEEE Trans. Green Commun. Netw. 2018, 2, 234-245. [CrossRef]

13. Feng, S.; Bai, T.; Hanzo, L. Joint power allocation for the multi-user NOMA-downlink in a power-line-fed VLC network. IEEE Trans. Veh. Technol. 2019, 68, 5185-5190. [CrossRef]

14. Ma, H.; Lampe, L.; Hranilovic, S. Hybrid visible light and power line communication for indoor multiuser downlink. J. Opt. Commun. Netw. 2017, 9, 635-647. [CrossRef]

15. Song, J.; Ding, W.; Yang, F.; Yang, H.; Yu, B.; Zhang, H. An indoor broadband broadcasting system based on PLC and VLC. IEEE Trans. Broadcasting 2015, 61, 299-308. [CrossRef]

16. Gao, S.; Zhang, J.; Yang, H. A reference channel model of indoor integrated power line communication and VLC system. Electr. Power Inf. Commun. Technol. 2018, 40, 1062-1066.

17. Song, J.; Liu, S.; Zhou, G.; Yu, B.; Ding, W.; Yang, F.; Zhang, H.; Zhang, X.; Amara, A. A cost-effective approach for ubiquitous broadband access based on hybrid PLC-VLC system. In IEEE International Symposium on Circuits and Systems (ISCAS); IEEE: Montreal, QC, Canada, 2016.

18. Chen, M.; Feng, Y.; You, S.; Wang, Y.; Wang, Z.; Chen, J. Performance analysis of channel estimation for hybrid PLC\&VLC system. Study Opt. Commun. 2017, 43, 3-6.

19. Ding, W.; Yang, F.; Yang, H.; Wang, J.; Wang, X.; Zhang, X. A hybrid power line and visible light communication system for indoor hospital applications. Comput. Ind. 2015, 68, 170-178. [CrossRef]

20. Ma, X.; Gao, J.; Yang, F.; Ding, W.; Yang, H.; Song, J. Integrated power line and visible light communication system compatible with multi-service transmission. IET Commun. 2017, 11, 104-111. [CrossRef]

21. Gao, J.; Yang, F.; Ding, W. Novel Integrated power line and visible light communication system with bit division multiplexing. In Proceedings of the 2015 International Wireless Communications and Mobile Computing Conference (IWCMC), Dubrovnik, Croatia, 24-28 August 2015; IEEE: Piscataway, NJ, USA, 2015.

22. Yang, L.; Li, J.; Zhang, J. Hybrid visible light communications (VLC) and PLC system. In Proceedings of the Opto-Electronics and Communications Conference (OECC) and Photonics Global Conference (PGC), Singapore, 31 July-4 August 2017.

23. Liu, H.; Zhu, P.; Chen, Y.; Huang, M. Power allocation for downlink hybrid power line and visible light communication system. IEEE Access 2020, 8, 24145-24152. [CrossRef] 
24. Pittolo, A.; Tonello, A.M.; Versolatto, F. Performance of MIMO PLC in measured channels affected by correlated noise. In Proceedings of the 18th IEEE International Symposium on Power Line Communications and Its Applications, Glasgow, UK, 12 May 2014; pp. 261-265.

25. Gao, S.; Zhang, J.; Song, J.; Yang, H.; Chu, X. Random channel generator of the integrated power line communication and visible light communication. In Proceedings of the 2017 IEEE International Symposium on Power Line Communications and its Applications (ISPLC), Madrid, Spain, 3-5 April 2017; pp. 1-7.

26. Zimmermann, M.; Dostert, K. A multipath model for the powerline channel. IEEE Trans. Commun. 2002, 50, 553-559. [CrossRef]

27. Ma, H.; Lampe, L.; Hranilovic, S. Integration of indoor visible light and power line communication systems. In Proceedings of the IEEE International Symposium on Power Line Communications \& Its Applications, Vancouver, BC, Canada, 21-24 October 2013; IEEE: Piscataway, NJ, USA, 2013.

28. Ma, H.; Lampe, L.; Hranilovic, S. Subcarrier allocation in hybrid visible light and power line communication system. In Proceedings of the IEEE International Symposium on Circuits \& Systems, Montreal, QC, Canada, 22-25 May 2016; IEEE: Piscataway, NJ, USA, 2016.

29. Kuhn, M.; Berger, S.; Hammerstrom, I.; Wittneben, A. Power line enhanced cooperative wireless communications. IEEE J. Sel. Areas Commun. 2006, 24, 1401-1410. [CrossRef]

30. Ndjiongue, A.R.; Ferreira, H.C.; Shongwe, T. Inter-building PLC-VLC integration based on PSK and CSK techniques. In Proceedings of the International Symposium on Power Line Communications \& Its Applications, Bottrop, Germany, 20-23 March 2016; IEEE: Piscataway, NJ, USA, 2016.

31. Nlom, S.M.; Ouahada, K.; Ndjiongue, A.R.; Ferreira, H.C. Evaluation of the SFSK-OOK integrated PLC-VLC system under the influence of sunlight. In Proceedings of the International Symposium on Networks, Computers and Communications (ISNCC), Marrakech, Morocco, 16-18 May 2017; IEEE: Piscataway, NJ, USA, 2017.

32. Ndjiongue, A.R.; Ferreira, H.C.; Ouahada, K.; Vinck, A.J.H. Low-complexity SOCPBFSK-OOK interface between PLC and VLC channels for low data rate transmission applications. In Proceedings of the IEEE International Symposium on Power Line Communications \& Its Applications, Glasgow, Scotland, 20 March-2 April 2014; IEEE: Piscataway, NJ, USA, 2014; pp. 226-231.

33. Komine, T.; Haruyama, S.; Nakagawa, M. Performance evaluation of narrowband OFDM on integrated system of power line communication and visible light wireless communication. In Proceedings of the 2006 1st International Symposium on Wireless Pervasive Computing, Phuket, Thailand, 16-18 January 2006; pp. 6-11.

34. Song, J.; Ding, W.; Yang, F.; Yang, H.; Wang, J.; Wang, X.; Zhang, X. Indoor hospital communication systems: An integrated solution based on power line and visible light communication. In Proceedings of the IEEE Faible Tension Faible Consommation, Monaco, Monaco, 4-6 May 2014; pp. 1-6.

35. Alavi, S.E.; Rezaie, H.; Supat, A.S.M. Application of OFDM on integrated system of visible free space optic with PLC. In Proceedings of the IEEE Asia-Pacific Conference on Applied Electromagnetics, Port Dickson, Malaysia, 9-11 November 2010; pp. 1-5.

36. Ma, H.; Lampe, L.; Hranilovic, S. Integration of indoor visible light and power line communication systems. In Proceedings of the 2013 IEEE 17th International Symposium on Power Line Communications and Its Applications, Johannesburg, South Africa, 24-27 March 2013; pp. 291-296.

37. Gheth, W.; Rabie, K.M.; Adebisi, B.; Ijaz, M.; Harris, G. Performance analysis of integrated power-line/visible-light communication systems with AF relaying. In Proceedings of the 2018 IEEE Global Communications Conference (GLOBECOM), Abu Dhabi, United Arab Emirates, 9-13 December 2018; pp. 1-6.

38. Gheth, W.; Rabie, K.M.; Adebisi, B.; Ijaz, M.; Harris, G. On the performance of DF-based power-line/visible-light communication systems. In Proceedings of the 2018 International Conference on Signal Processing and Information Security (ICSPIS), Dubai, United Arab Emirates, 7-8 November 2018; pp. 1-4.

39. Basnayaka, D.A.; Haas, H. Hybrid RF and VLC systems: Improving user data rate performance of VLC systems. In Proceedings of the 2015 IEEE 81st Vehicular Technology Conference (VTC Spring), Glasgow, UK, 1-14 May 2015; pp. 1-5.

40. Kashef, M.; Ismail, M.; Abdallah, M.; Qarage, K.; Serpedin, E. Energy efficient resource allocation for mixed RF/VLC heterogeneous wireless networks. IEEE J. Sel. Areas Commun. 2016, 34, 883-893. [CrossRef] 
41. Kubjana, M.D.; Shongwe, T.; Ndjiongue, A.R. Hybrid PLC-VLC based on ACO-OFDM. In Proceedings of the 2018 International Conference on Intelligent and Innovative Computing Applications (ICONIC), Plaine Magnien, Mauritius, 6-7 December 2018; IEEE: Piscataway, NJ, USA, 2018.

42. Kubjana, M.D.; Ndjiongue, A.R.; Shongwe, T. Impulsive noise evaluation on PLC-VLC based on DCO-OFDM. In Proceedings of the 11th International Symposium on Communication Systems, Networks \& Digital Signal Processing (CSNDSP), Budapest, Hungary, 18-20 July 2018.

43. Mapfumo, I.; Shongwe, T. A comparison of FFT and DHT hybrid PLC-VLC systems based on ACO-OFDM in impulsive noise. In Proceedings of the 30th International Conference Radioelektronika (RADIOELEKTRONIKA), Bratislava, Slovakia, 15-16 April 2020; pp. 1-7.

44. Kizilirmak, R.C.; Rowell, C.R.; Uysal, M. Non-orthogonal multiple access (NOMA) for indoor visible light communications. In Proceedings of the 4th International Workshop on Optical Wireless Communications (IWOW), Istanbul, Turkey, 7-8 September 2015; pp. 98-101.

45. Yin, L.; Popoola, W.O.; Wu, X.; Haas, H. Performance evaluation of non-orthogonal multiple access in visible light communication. IEEE Trans. Commun. 2016, 64, 5162-5175. [CrossRef]

46. Lin, B.; Tang, X.; Ghassemlooy, Z. Optical power domain NOMA for visible light communications. IEEE Wirel. Commun. Lett. 2019, 8, 1260-1263. [CrossRef]

47. Liu, X.; Wang, Y.; Zhou, F.; Hu, R.Q. BER Analysis for NOMA-Enabled Visible Light Communication Systems with M-PSK. In Proceedings of the 2018 10th International Conference on Wireless Communications and Signal Processing (WCSP), Hangzhou, China, 18-20 October 2018; IEEE: Piscataway, NJ, USA, 2018.

48. Ye, Q.; Rong, B.; Chen, Y.; AL-Shalash, M.; Caramanis, C.; Andrews, J.G. User association for load balancing in heterogeneous cellular networks. IEEE Trans. Wirel. Commun. 2013, 12, 2706-2716. [CrossRef]

49. Lee, J.W.; Mazumdar, R.R.; Shroff, N.B. Joint resource allocation and base-station assignment for the downlink in CDMA networks. IEEE/ACM Trans. Netw. 2006, 14, 1-14. [CrossRef]

50. Amirshahi, P.; Kavehrad, M. Broadband access over medium and low voltage power-lines and use of white light emitting diodes for indoor communications. In Proceedings of the Consumer Communications and Networking Conference, Barcelona, Spain, 23-29 April 2006; IEEE: Piscataway, NJ, USA, 2006; pp. 897-901.

51. Yoon, S.G.; Jang, S.; Kim, Y.H.; Bahk, S. Opportunistic routing for smart grid with power line communication access networks. IEEE Trans. Smart Grid 2014, 5, 303-311. [CrossRef]

52. Ferreira, H.C.; Lampe, L.; Newbury, J.; Swart, T.G. Power Line Communications: Theory and Applications for Narrowband and Broadband Communications over Power Lines; Wiley: Hoboken, NJ, USA, 2010; p. 11.

53. Anatory, J.; Theethayi, N. Broadband Power-line Communication Systems: Theory E Applications; WIT Press: Cambridge, MA, USA, 2010; p. 1.

54. Luján, R.D.; Edenor, S.A.A. Harmonics disturbances survey in distribution networks. In Proceedings of the 21st International Conference on Electricity Distribution, Frankfurt, Germany, 6-9 June 2011; pp. 1-4.

55. Liu, Q.; Sun, D.; Zang, Z.; Wang, X.; Zhang, S.; Liu, J.; CG. Co., Ltd. Design of visible light and power line communication protocol for electric power tunnel inspection. Autom. Instrum. 2018, 11, 133-137.

56. $\mathrm{Xu}, \mathrm{Z}$. An autonomous intelligent UAV line inspection system with LIDAR navigation. Zhejiang Electr. Power 2017, 36, 44-47.

57. Zhang, J.; Yang, Y.; Liu, B.; Chen, X.; Chen, H. Design of home network based on visible light communication and power line carrier communication. Laser Optoelectron. Prog. 2011, 48, 100604. [CrossRef]

58. Reddy, Y.S.; Panda, M.; Dubey, A.; Kumar, A.; Panigrahi, T.; Rabie, K.M. Optimisation of indoor hybrid PLC/VLC/RF communication systems. IET Commun. 2020, 14, 117-126. [CrossRef]

59. Sarafi, A.M.; Stavropoulos, G.I.; Cottis, P.G. Hybrid wireless-broadband over power lines: A promising broadband solution in rural areas. IEEE Commun. Mag. 2009, 47, 140-147. [CrossRef]

60. Pan, Q.W.; Kathnaur, A. Power-line networks to extend ranges of $2.4 \mathrm{GHz}$ wireless communications inside multi-storey buildings. In Proceedings of the IEEE Radio and Wireless Symposium (RWS), New Orleans, LA, USA, 10-14 January 2010; pp. 621-624.

61. Güzelgöz, S.; Çelebi, H.B.; Arsian, H. Analysis of a multi-channel receiver: Wireless and PLC reception. In Proceedings of the European Signal Processing Conference, Aalborg, Denmark, 23-27 August 2010; pp. 1106-1110. 
62. Gheth, W.; Rabie, K.M.; Adebisi, B.; Ijaz, M.; Harris, G.; Alfitouri, A. Hybrid power-line/wireless communication systems for indoor applications. In Proceedings of the 11th International Symposium on Communication Systems, Networks \& Digital Signal Processing (CSNDSP), Budapest, Hungary, 18-20 July 2018; pp. 1-6.

63. Gupta, A.; Sharma, N.; Garg, P.; Alouini, M.S. Cascaded FSO-VLC communication system. IEEE Wirel. Commun. Lett. 2017, 6, 810-813. [CrossRef]

64. Chen, M.; You, S.; Wang, Y.; Wang, Z.; Bian, S.; Chen, H.; Ding, J.; Wu, H.; Shi, R.; Zhou, S.; et al. Performance analysis of pilot-based OFDM channel estimation for hybrid PLC\&VLC system. In Proceedings of the 15th International Conference on Optical Communications and Networks (ICOCN), Hangzhou, China, 24-27 September 2016; IEEE: Piscataway, NJ, USA, 2016.

65. Ndjiongue, A.R.; Shongwe, T.; Ferreira, H.C.; Ngatched, T.M.N.; Vinck, A.J.H. Cascaded PLC-VLC channel using OFDM and CSK techniques. In Proceedings of the IEEE Global Communications Conference (GLOBECOM), San Diego, CA, USA, 6-10 December 2015; IEEE: Piscataway, NJ, USA, 2015.

66. Kashef, M.; Abdallah, M.; Al-Dhahir, N.; Qaraqe, K. On the impact of PLC backhauling in multi-user hybrid VLC/RF communication systems. In Proceedings of the IEEE Global Communications Conference (GLOBECOM), Washington, DC, USA, 4-8 December 2016; pp. 1-6.

67. Narmanlioglu, O.; Kizilirmak, R.C.; Uysal, M. Relay-assisted OFDM-based visible light communications over multipath channels. In Proceedings of the 2015 17th International Conference on Transparent Optical Networks (ICTON), Budapest, Hungary, 5-9 July 2015; pp. 1-4.

68. Jani, M.; Garg, P.; Gupta, A. Modeling and outage analysis of DF relay assisted mixed PLC-VLC system. In Proceedings of the Twenty Fourth National Conference on Communications (NCC), Hyderabad, India, 25-28 February 2018; IEEE: Piscataway, NJ, USA, 2018.

69. Jani, M.; Garg, P.; Gupta, A. Performance analysis of a co-operative PLC/VLC system with multiple access points for indoor broadcasting. Aeu-Int. J. Electron. Commun. 2019, 103, 64-73. [CrossRef]

70. Jani, M.; Garg, P.; Gupta, A. Outage analysis of an asymmetric dual hop PLC-VLC system for indoor broadcastin. In Proceedings of the National Conference on Communications (NCC), Bangalore, India, 20-23 February 2019; IEEE: Piscataway, NJ, USA, 2019.

71. Kashef, M.; Torky, A.; Abdallah, M.; Aldhahir, N.; Qaraqe, K. On the achievable rate of a hybrid PLC/VLC/RF communication system. In Proceedings of the IEEE Global Communications Conference, San Diego, CA, USA, 6-10 December 2015; IEEE: Piscataway, NJ, USA, 2015.

72. Deka, R.; Verma, A.; Anees, S. Performance analysis of decode-and-forward based hybrid RF/FSO-VLC system. In Proceedings of the IEEE International Conference on Advanced Networks and Telecommunications Systems (ANTS), GOA, India, 16-19 December 2019.

73. Jani, M.; Garg, P.; Gupta, A. On the performance of a cooperative PLC-VLC indoor broadcasting system consisting of mobile user nodes for IoT networks. IEEE Trans. Broadcasting 2020, 1-10. [CrossRef]

74. Saldaña-González, A.E.; Sumper, A.; Aragüés-Peñalba, M.; Smolnikar, M. Advanced distribution measurement technologies and data applications for smart grids: A review. Energies 2020, 13, 3730. [CrossRef]

75. Della Giustina, D.; Rinaldi, S. Hybrid communication network for the smart grid: Validation of a field test experience. IEEE Trans. Power Deliv. 2015, 30, 2492-2500. [CrossRef]

76. Sharma, K.; Saini, L.M. Power-line communications for smart grid: Progress, challenges, opportunities and status. Renew. Sustain. Energy Rev. 2017, 67, 704-751. [CrossRef]

77. Rosolem, J.B.; Penze, R.S.; Floridia, C.; Dini, D.C.; Valadares, J.E.F. FSO applied to optical fiber sensing: A field test demonstration. In Proceedings of the SBMO/IEEE MTT-S International Microwave and Optoelectronics Conference (IMOC), Águas de Lindoia, SP, Brazil, 27-30 August 2017; IEEE: Piscataway, NJ, USA, 2017.

78. Rosolem, J.B.; Bassan, F.R.; Penze, R.S.; Leonardi, A.A.; Fracarolli, J.P.V.; Floridia, C. Optical sensing in high voltage transmission lines using power over fiber and free space optics. Opt. Fiber Technol. 2015, 26, 180-183. [CrossRef]

79. Floridia, C.; Rosolem, J.B.; Leonardi, A.A.; Hortencio, C.A.; Fonseca, R.F.; Moreira, R.O.C.; Souza, G.C.L.; Melo, A.L.; Nascimento, C.A.M. Temperature sensing in high voltage transmission lines using fiber Bragg grating and free-space-optics. In SPIE Defense, Security, and Sensing; International Society for Optics and Photonics: Bellingham, DC, USA, 2013. 
80. Rosolem, J.B.; Dini, D.C.; Penze, R.S.; Floridia, C.; Leonardi, A.A.; Loichate, M.D.; Durelli, A.S. Fiber optic bending sensor for water level monitoring: Development and field test: A review. IEEE Sens. J. 2013, 13, 4113-4120. [CrossRef]

81. Sun, D.; Xu, D.; Gong, T.; Song, J.; Liu, R. The design of VLC system for intelligent patrol inspection. Distrib. Util. 2016, 11, 27-30.

82. Gao, Z.; Chen, D.; Wu, H.-C. Energy loss minimization for wireless power transfer based energy redistribution in WSNs. IEEE Trans. Veh. Technol. 2019, 68, 12271-12285. [CrossRef]

83. Pandey, G.; Narsa, R.T. Power flow study of grid connected bidirectional WPT systems for EV application. In Proceedings of the IEEE International Conference on Power Electronics, Smart Grid and Renewable Energy (PESGRE), Cochin, India, 2-4 January 2020.

84. Zhou, Y.; Liu, C.; Huang, Y. Wireless power transfer for implanted medical application: A review. Energies 2020, 13, 2837. [CrossRef]

85. Abdullahi, Q.S.; Joshi, R.; Podilchak, S.K.; Khan, S.R.; Chen, M.; Rooney, J.; Rooney, J.; Sun, D.; Desmulliez, M.P.Y.; Georgiadis, A.; et al. Design of a wireless power transfer system for assisted living applications. Wirel. Power Transf. 2019, 6, 41-56. [CrossRef]

86. Nguyen, M.T.; Nguyen, C.V.; Truong, L.H.; Le, A.M.; Quyen, T.V.; Masaracchia, A.; Teague, K.A. Electromagnetic field based WPT technologies for UAVs: A comprehensive survey. Electronics 2020, 9, 461. [CrossRef]

87. Tang, Y.; Chen, Y.; Madawala, U.K.; Thrimawithana, D.J.; Ma, H. A new controller for bidirectional wireless power transfer systems. IEEE Trans. Power Electron. 2018, 38, 9076-9087. [CrossRef]

88. Mohamed, A.A.S.; Berzoy, A.; Mohammed, O.A. Experimental validation of comprehensive steady-state analytical model of bidirectional WPT system in EVs applications. IEEE Trans. Veh. Technol. 2017, 66, 5584-5594. [CrossRef]

89. Thrimawithana, D.J.; Madawala, U.K.; Shi, Y. Design of a bi-directional inverter for a wireless V2G system. In Proceedings of the IEEE International Conference on Sustainable Energy Technologies (IC-SET), Kandy, Sri Lanka, 6-9 December 2010; pp. 1-5.

90. Raavi, S.; Arigong, B.; Zhou, R.; Jung, S.; Jin, M.; Zhang, H.; Kim, H. An optical wireless power transfer system for rapid charging. In Proceedings of the Texas Symposium on Wireless and Microwave Circuits and Systems (WMCS), Waco, TX, USA, 4-5 April 2013; pp. 1-4.

91. Kurs, A.; Karalis, A.; Moffatt, R.; Joannopoulos, J.D.; Fisher, P.; Soljacic, M. Wireless power transfer via strongly coupled magnetic resonances. Science 2007, 317, 83-86. [CrossRef]

92. Han, Z.; Kim, I.D.; Lu, X.; Niyato, D.; Wang, P. Wireless charging technologies: Fundamentals standards and network applications. IEEE Commun. Surv. Tutor. 2016, 18, 1413-1452.

93. Ho, S.L.; Wang, J.; Fu, W.N.; Sun, M. A comparative study between novel Witricity and traditional inductive magnetic coupling in wireless charging. IEEE Trans. Magn. 2011, 45, 1522-1525. [CrossRef]

94. Kline, M.; Izyumin, I.; Boser, B.; Sanders, S. Capacitive power transfer for contactless charging. In Proceedings of the Twenty-Sixth Annual IEEE Applied Power Electronics Conference and Exposition (APEC), Fort Worth, TX, USA, 6-11 March 2011; p. 1398.

95. Shinohara, N. Beam efficiency of wireless power transmission via radio waves from short range to long range. J. Korean Inst. Electromagn. Eng. Sci. 2010, 10, 224. [CrossRef]

96. Yedavalli, P.S.; Riihonen, T.; Wang, X.; Rabaey, J.M. Far-field RF wireless power transfer with blind adaptive beamforming for Internet of Things devices. IEEE Access 2017, 5, 1743-1752. [CrossRef]

97. Rhee, D.-H.; Kim, S.-M. Unknown. J. Korea Inst. Electro. Commun. Sci. 2016, 11, 1219. [CrossRef]

98. Kim, S.-M. Wireless optical energy transmission using optical beamforming. Opt. Eng. 2013, 52, 1-5. [CrossRef]

99. Haydaroglu, I.; Mutlu, S. Optical power delivery and data transmission in a wireless and batteryless microsystem using a single light emitting diode. J. Microelectromechanical Syst. 2015, 24, 155-165. [CrossRef]

100. Fakidis, J.; Videv, S.; Kucera, S.; Claussen, H.; Haas, H. Indoor optical wireless power transfer to small cells at nighttime. J. Lightwave Technol. 2016, 34, 3236-3258. [CrossRef]

101. Pani, C.; Ray, O.; Ghosh, A.; Qadir, Z.; Sarkar, M.M.; Reja, W. Wireless power transfer using free space photonics. In Proceedings of the 2nd International Conference on Advances in Computing, Communication, \& Automation (Fall), Bareilly, India, 30 September-1 October 2016; pp. 1-5. 
102. Saha, A.; Iqbal, S.; Karmaker, M.; Zinnat, S.F.; Ali, M.T. A wireless optical power system for medical implants using low power near-IR laser. In Proceedings of the Annual International Conference of the IEEE Engineering in Medicine and Biology Society (EMBC), Seogwipo, Korea, 11-15 July 2017; pp. 1978-1981.

103. Kim, S.-M.; Choi, J.; Jung, H. Experimental demonstration of underwater optical wireless power transfer using a laser diode. Chin. Opt. Lett. 2018, 10, 080101.

104. Fakidis, J.; Ijaz, M.; Kucera, S.; Claussen, H.; Haas, H. On the design of an optical wireless link for small cell backhaul communication and energy harvesting. In Proceedings of the IEEE 25th Annual International Symposium Personal Indoor Mobile Radio Communication, Washington, DC, USA, 4-8 December 2014; pp. 58-62.

105. Fakidis, J.; Kucera, S.; Claussen, H.; Haas, H. On the design of a free space optical link for small cell backhaul communication and power supply. In Proceedings of the IEEE International Conference Communication Workshop, London, UK, 15-17 April 2015; pp. 1428-1433.

106. Tang, J.; Miyamoto, T. Numerical and experimental analysis of power generation characteristics in beam direction control of optical wireless power transmission with mirror. In Proceedings of the 24th Microoptics Conference (MOC2019), Toyama, Japan, 17-20 November 2019; pp. 164-165.

107. Tang, J.; Matsunaga, K.; Miyamoto, T. Numerical analysis of power generation characteristics in beam irradiation control of indoor OWPT system. Opt. Rev. 2020, 27, 170-176. [CrossRef]

108. Khan, S.R.; Pavuluri, S.K.; Cummins, G.; Desmulliez, M.P.Y. Wireless power transfer techniques for implantable medical devices: A review. Sensors 2020, 20, 3487. [CrossRef]

109. Mujeeb, U.; Rahman, M.; Adalian, D.; Chang, C.-F.; Scherer, A. Optical power transfer and communication methods for wireless implantable sensing platforms. J. Biomed. Opt. 2015, 20, 95012. [CrossRef] [PubMed]

110. Goto, K.; Nakagawa, T.; Nakamura, O.; Kawata, S. An implantable power supply with an optically rechargeable lithium battery. IEEE Trans. Biomed. Eng. 2001, 48, 830-833. [CrossRef] [PubMed]

111. Santis, M.D.; Cacciotti, I. Wireless implantable and biodegradable sensors for postsurgery monitoring: Current status and future perspectives. Nanotechnology 2020, 35, 252001. [CrossRef] [PubMed]

112. Dregely, D.; Lindfors, K.; Lippitz, M.; Engheta, N.; Totzeck, M.; Giessen, H. Imaging and steering an optical wireless nanoantenna link. Nat. Commun. 2014, 5, 4354. [CrossRef] [PubMed]

113. Fan, X.; Gao, L.; Mo, X.; Zhao, Q.; Jia, E. Overview of research status and application of wireless power transmission technology. Trans. China Electrotech. Soc. 2019, 34, 1353-1380.

114. Luo, W.; Dong, W.; Yang, H.; Xu, P.; Tang, Z. Development trend of high power lasers. Laser Infrared 2013, 43, 845-852.

115. Liu, Q.; Wu, J.; Xia, P.; Zhao, S.; Yang, Y.; Chen, W.; Hanzo, L. Charging unplugged: Will distributed laser charging for mobile wireless power transfer work? IEEE Veh. Technol. Mag. 2016, 11, 36-45. [CrossRef]

116. Motohiro, T.; Takeda, Y.; Ito, H.; Hasegawa, K.; Ikesue, A.; Ichikawa, T.; Higuchi, K.; Ichiki, A.; Mizuno, S.; Yamada, N.; et al. Concept of the solar-pumped laser-photovoltaics combined system and its application to laser beam power feeding to electric vehicles. Jpn. J. Appl. Phys. 2017, 56, 08MA07. [CrossRef]

117. Nguyen, D.H. Optical wireless power transfer for moving objects as a life-support technology. In Proceedings of the 2nd Global Conference on Life Sciences and Technologies, Kyoto, Japan, 10-12 March 2020; pp. 405-408.

118. Wi-Charge. Available online: https://wi-charge.com (accessed on 13 September 2020).

119. Japan Aerospace Exploration Agency. Research on Laser Wireless Power Transmission Technology. Available online: http://www.kenkai.jaxa.jp/eng/research/ssps/ssps-lssps.html (accessed on 13 September 2020).

120. Fraunhofer Institute for Solar Energy Systems ISE, Germany. Science Blog. Available online: https: //blog.innovation4e.de/en/2016/09/12/power-by-light (accessed on 13 September 2020).

121. Jin, M.H.C.; Pierce, J.M.; Lambiotte, J.C.; Fite, J.D.; Marshall, J.S.; Huntley, M.A. Underwater free-space optical power transfer: An enabling technology for remote underwater intervention. In Proceedings of the Offshore Technology Conference, Houston, TX, USA, 30 April-3 May 2018; pp. 1-9.

122. Kobe University. Solar cell design with over 50\% energy-conversion efficiency. In PHYSE ORG, Technology, Energy \& Green Tech; Kobe University: Kobe, Japan, 2017.

123. Lumb, M.P.; Mack, S.; Schmieder, K.J.; González, M.; Bennett, M.F.; Scheiman, D.; Meitl, M.; Fisher, B.; Burroughs, S.; Lee, K.T.; et al. GaSb-Based Solar Cells for Full Solar Spectrum Energy Harvesting. Adv. Energy Mater. 2017, 7, 1700345. [CrossRef]

124. Zhu, P. Solar Cell with a Photoelectric Conversion Efficiency of $44.5 \%$. Available online: http://blog.sciencenet. cn/blog-212210-1066142.html (accessed on 13 September 2020). 
125. WIPO IP PORTAL. Available online: http://patentscope2.wipo.int/search/en/result.jsf?_vid=JP1-KDIOPI61891 (accessed on 13 September 2020).

126. Hashmat, R.; Pagani, P.; Zeddam, A.; Chonavel, T. MIMO communications for inhome PLC networks: Measurements and results up to $100 \mathrm{MHz}$. In Proceedings of the IEEE International Symposium on Power Line Communications \& Its Applications, Rio de Janeiro, Brazil, 28-31 March 2010; IEEE: Piscataway, NJ, USA, 2010.

127. Schneider, D.; Schwager, A.; Speidel, J.; Dilly, A. Implementation and results of a MIMO PLC feasibility study. In Proceedings of the Power Line Communications and Its Applications (ISPLC), Udine, Italy, 3-6 April 2011.

128. Cano, C.; Pittolo, A.; Malone, D.; Lampe, L.; Tonello, A.M.; Dabak, A.G. State-of-the-art in power line communications: From the applications to the medium. IEEE J. Sel. Areas Commun. 2016, 34, 1935-1952. [CrossRef]

129. Laguna, G.; Barron, R. Survey on indoor power line communication channel modeling. In Proceedings of the Power Line Communications and Its Applications (ISPLC), Electronics Robotics and Automotive Mechanics Conference, Morelos, Mexico, 30 September-3 October 2008; IEEE: Piscataway, NJ, USA, 2008.

130. Zimmermann, M.; Dostert, K. Analysis and modeling of impulsive noise in broad-band powerline communications. IEEE Trans. Electromagn. Compat. 2002, 44, 249-258. [CrossRef]

131. Ding, J.; Chih-Lin, I.; Chen, X.; Lai, H. Asymmetrical emission beams based visible light communication access points design. In Proceedings of the 28th Wireless and Optical Communications Conference (WOCC), Beijing, China, 9-10 May 2019; IEEE: Piscataway, NJ, USA, 2019.

132. Ding, J.; Chih-Lin, I.; Zhang, H.; Chen, X.; Lai, H. Cells planning of VLC networks using non-circular symmetric optical beam. In Proceedings of the 2019 IEEE International Conference on Communications (ICC), Shanghai, China, 20-24 May 2019; IEEE: Piscataway, NJ, USA, 2019.

133. Ding, J.; Chih-Lin, I.; Xu, Z. Indoor optical wireless channel characteristics with distinct source radiation patterns. IEEE Photonics J. 2016, 8, 1-15. [CrossRef]

134. You, C.; Hu, Q.; Li, S.; Ma, Y.; Chen, H. Light source distribution for visible light communication in coal mine working face based on positioning and power co-optimization. Chin. J. Lasers 2019, 46, 0406001.

135. Chen, Y.; Shen, Q.; Liu, H. Optimization algorithm of receiver optical power uniformity in indoor visible light communication. Chin. J. Lasers 2018, 45, 0506003. [CrossRef]

136. Kim, S.M.; Baek, M.W.; Nahm, S.H. Visible light communication using TDMA optical beamforming. Eurasip J. Wirel. Commun. Netw. 2017, 2017, 1-6. [CrossRef]

137. Campi, T.; Cruciani, S.; Maradei, F.; Feliziani, M. Near-field reduction in a wireless power transfer system using LCC compensation. IEEE Trans. Electromagn. Comput. 2017, 59, 686-694. [CrossRef]

138. Campi, T.; Cruciani, S.; Santis, V.D.; Feliziani, M. EMF safety and thermal aspects in a pacemaker equipped with a wireless power transfer system working at low frequency. IEEE Trans. Microw. Theory Tech. 2016, 64, 375-382. [CrossRef]

139. Jawad, A.M.; Nordin, R.; Gharghan, S.K.; Jawad, H.M.; Ismail, M. Opportunities and challenges for near-field wireless power transfer: A review. Energies 2017, 10, 1022. [CrossRef]

140. Nair, V.V.; Choi, J. An efficiency enhancement technique for a wireless power transmission system based on a multiple coil switching technique. Energies 2016, 9, 156. [CrossRef]

141. Feliziani, M.; Campi, T.; Cruciani, S.; Maradei, F.; Grasselli, U.; Macellari, M.; Schirone, L. Robust LCC compensation in wireless power transfer with variable coupling factor due to coil misalignment. In Proceedings of the IEEE 15th International Conference on Environment and Electrical Engineering (EEEIC), Rome, Italy, 10-13 June 2015; pp. 1181-1186.

(C) 2020 by the authors. Licensee MDPI, Basel, Switzerland. This article is an open access article distributed under the terms and conditions of the Creative Commons Attribution (CC BY) license (http://creativecommons.org/licenses/by/4.0/). 